\title{
Synthesis Toward and Stereochemistry Assignment of Clathsterol: Exploring Diverse Strategies to Polyoxygenated Sterols
}

Tao Zhou, Feng Feng, Yong Shi,* and Wei-Sheng Tian*

Key Laboratory of Synthetic Chemistry of Natural Substances, Shanghai Institute of Organic Chemistry, Chinese Academy of Sciences, 345 Lingling Road, Shanghai 200032, China

Email: shiong81@sioc.ac.cn; wstian@sioc.ac.cn

\section{Table of Content}

General Methods

Experimental Sections

NMR Spectra 


\section{General Methods}

All reactions sensitive to air or moisture were performed in flame-dried round bottom flasks with rubber septum under a positive pressure of argon or nitrogen atmosphere, unless otherwise noted. Air and moisture-sensitive liquids and solutions were transferred via syringe and stainless steel cannula. Tetrahydrofuran (THF) and diethyl ether $\left(\mathrm{Et}_{2} \mathrm{O}\right)$ were distilled from sodium/benzophenone, methylene chloride (DCM), toluene, and 2,6-lutidine from calcium hydride, $N, N$-dimethylformamide (DMF) and dimethylsulfoxide from calcium hydride under reduced pressure, acetone from $\mathrm{K}_{2} \mathrm{CO}_{3}$ onto activated $3 \AA$ molecular sieves, others according to the standard procedures described in Purification of Laboratory Chemicals (2009). Yields refer to chromatographically and spectroscopically ( ${ }^{1} \mathrm{H}$ NMR) homogeneous materials, unless otherwise stated. Reactions were monitored by thin-layer chromatography (TLC) carried out on silica gel plates using UV light as visualizing agent and an ethanolic solution of phosphomolybic acid, and heat as developing agents. NMR spectra were recorded on Bruker DRX-400 instrument and calibrated using residual undeuterated solvent as an internal reference $\left[{ }^{1} \mathrm{H}\right.$ NMR: TMS (0.00), $\mathrm{CDCl}_{3}(7.26) ;{ }^{13} \mathrm{C} \mathrm{NMR}: \mathrm{CDCl}_{3}$ (77.16)]. The following abbreviations were used to explain the multiplicities: $\mathrm{s}=$ singlet, $\mathrm{d}=$ doublet, $\mathrm{t}=$ triplet, $\mathrm{q}=$ quartet, $\mathrm{br}=$ broad. 
We narrowed the eight possible isomer of clathsterol down to two isomers, $22 R, 23 R, 23 R$ - and $22 R, 23 R, 24 S$-, for reasons described below.

1. Most of the natural sterols that have 22,23-diol unit are $22 R, 23 R$-configured. We have searched some 100 such sterols and found all the 22,23-diol are (cis)-22R,23R-configured.

2. Treating clathsterol with $\mathrm{HCl}$ resulted the formation of $\mathbf{2}$ and the configuration at $\mathrm{C} 22$ of $\mathbf{2}$ was unambiguously assigned as $S$. Kashman and co-workers hesitated to deduce the stereochemistry of C22 in $\mathbf{1}$ because they were not sure of the mechanism of closure of the tetrahydrofuran ring. ${ }^{1}$ There could be a possibility of the participation of neighbor group but this possibility is very small. We believed the acid hydrolysis of $\mathbf{1}$ resulted in an invertion of the configuration at $\mathrm{C} 22$, therefore we deduced $\mathbf{1}$ is $22 R$-configured. Later, according to the procedure reported by Kashman, we transformed the $22 R$-configured $\mathbf{1 5}$ to 2 thus verified the above hypothesis.

3. We collected and examined the ${ }^{1} \mathrm{H}$ NMR data of some sterols and tried to discover some patterns. ${ }^{2}$ Brassinolide and its analogues have the biggest compound library and their NMR data were therefore used. Depicted in figure is the splitting patterns of their $\mathrm{C} 22-\mathrm{H}$ and $\mathrm{C} 23-\mathrm{H}$. Only the $22 R, 23 R$-diols have the splitting patterns that observed in clathsterol.

Observed ${ }^{1} \mathrm{H}$ NMR splitting patterns of $\mathrm{H} 22 \mathrm{~s} / \mathrm{H} 23 \mathrm{~s}$ of brassinolide analogues (the steroidal backbones are omitted for clarity)<smiles>CC(C)[C@H](C)[C@@H](O)[C@H](O)C(C)C</smiles>

not available<smiles>CC(C)[C@H](O)[C@@H](C)[C@H](O)C(C)C</smiles>

not available<smiles>CC(C)[C@H](C)[C@@H](O)[C@H](C)[C@H](O)C(C)C</smiles>

H22 dd, H23 dd

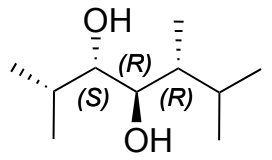

H22 br d, H23 br d<smiles>CC(C)[C@H](C)[C@@H](O)[C@H](C)O</smiles>

H22 dd, H23 br s

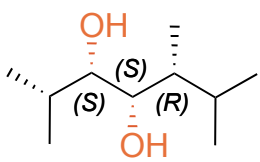

H22 m, H23 m

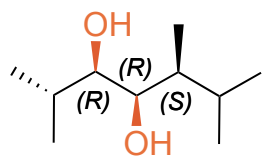

$\mathrm{H} 22 \mathrm{~d}, \mathrm{H} 23 \mathrm{~d} / \mathrm{dd}$

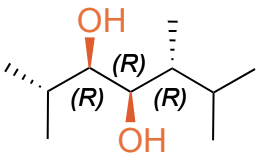

H22 d, H23 t/dd
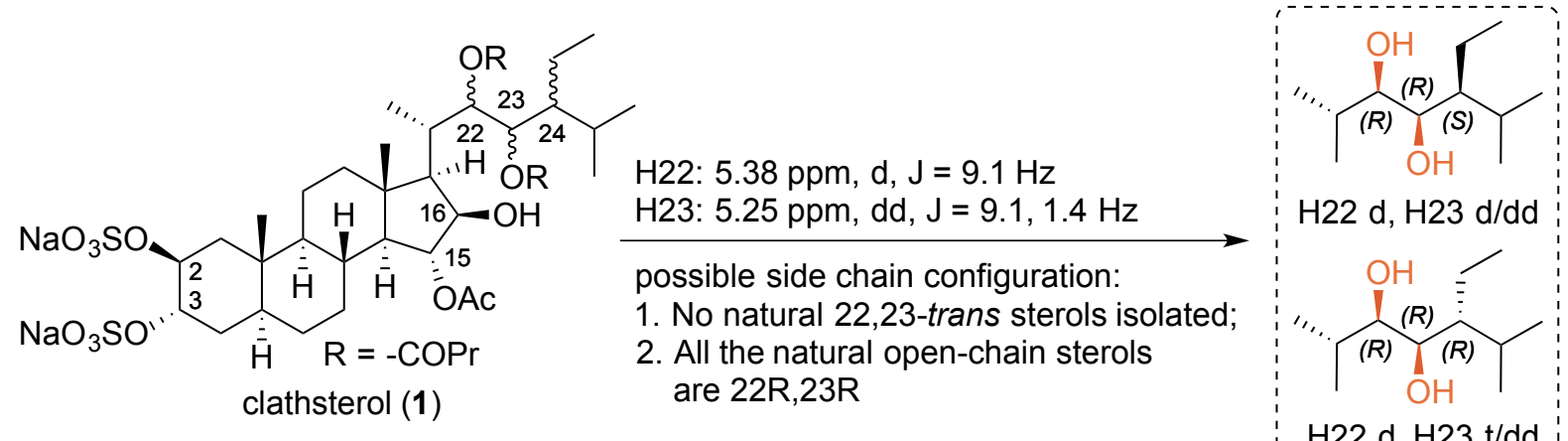

$\mathrm{H} 22 \mathrm{~d}, \mathrm{H} 23 \mathrm{t} / \mathrm{dd}$

\footnotetext{
${ }^{1}$ Rudi, A.; Yosief, T.; Loya, S.; Hizi, A.; Schleyer, M.; Kashman, Y. J. Nat. Prod. 2001, 64, 1451-1453.

2 The alkyl groups at $\mathrm{C} 24$ and substitution at C16 do not change the splitting patterns of $\mathrm{C} 22$ and C23, but would change their chemical shifts. The variations on AB rings of steroidal skeletons are remote from the side chains and do not have much influence on chemical shifts and splitting patterns.
} 
<smiles>C/C=C\C=C/C</smiles>

22: $3.56 \mathrm{ppm}, \mathrm{d}, \mathrm{J}=8.1$ 23: $3.71 \mathrm{ppm}, \mathrm{d}, \mathrm{J}=8.1$<smiles>C/C=C\C=C/C</smiles>

22: $3.70 \mathrm{ppm}, \mathrm{d}, \mathrm{J}=3.6$ 23: $3.40 \mathrm{ppm}, \mathrm{t}, \mathrm{J}=5.2$

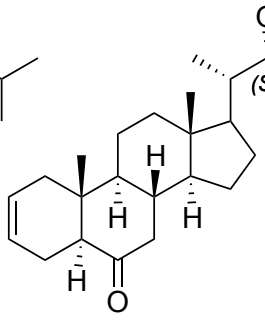

22 or 23 :

$3.45(\mathrm{dd}, \mathrm{J}=8.6,6.3)$ $3.68(\mathrm{br} \mathrm{s})$

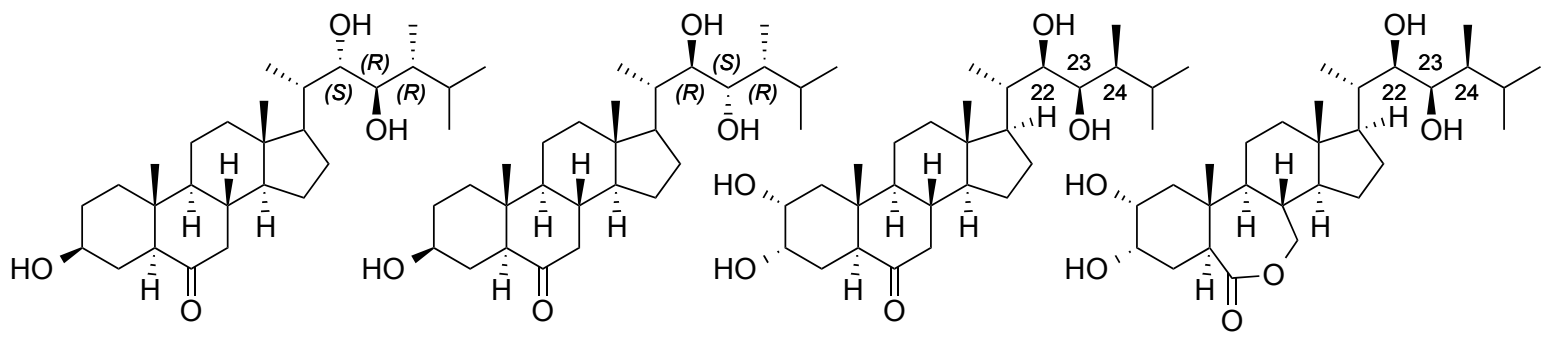

22: $3.75 \mathrm{ppm}, \mathrm{dd}, \mathrm{J}=8.4,2.622: 3.546 \mathrm{ppm}, \mathrm{br} \mathrm{d}, \mathrm{J}=8.5$ castasterone

23: 3.62 ppm, dd, J = 8.4, 4.2 23: 3.677 ppm, br d, J = 8.5 22: 3.52 ppm, d, J=8 Hz 23: $3.69 \mathrm{ppm}, \mathrm{d}, J=8 \mathrm{~Hz}$<smiles>CC[C@H](C(C)C)[C@H](O)[C@H](O)[C@@H](C)C1CCC2C3CCC4CC(O)CCC4(C)C3CCC21C</smiles>

22: $3.56 \mathrm{ppm}, \mathrm{d}, \mathrm{J}=9.0$ 23: $3.68 \mathrm{ppm}, \mathrm{d}, \mathrm{J}=8.4$

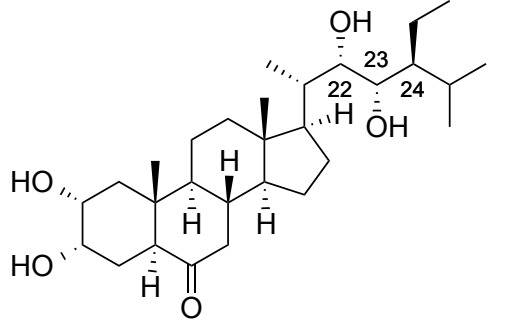

22,23: 3.59 ppm, m, $2 \mathrm{H}$<smiles>CC[C@H](C(C)C)[C@@H](O)[C@H](O)[C@@H](C)[C@H]1CC[C@H]2[C@@H]3OC(=O)C4CC[C@@H](O)C[C@]4(C)C3CC[C@@]21C</smiles>

22,23: $3.60 \mathrm{ppm}, \mathrm{m}, 2 \mathrm{H}$ 


\section{Experimental Sections}
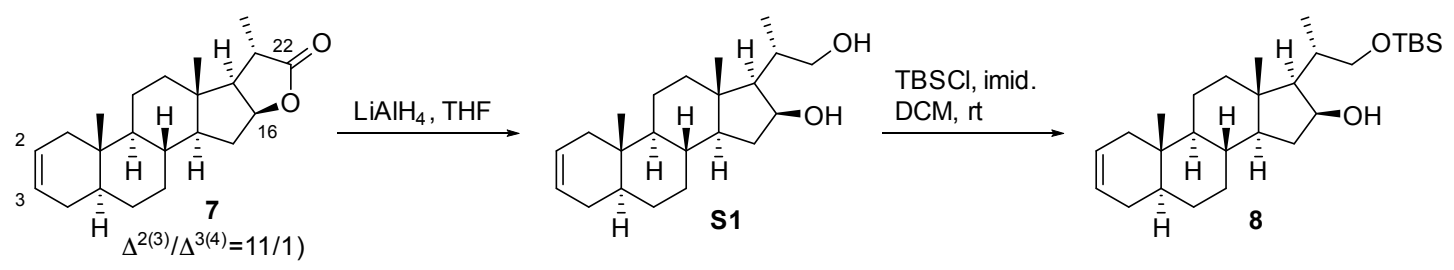

\section{Pregn-2,3-en-16,22-diol (S1)}

To a solution of lactone $7(29 \mathrm{~g}, 88 \mathrm{mmol})$ in anhydrous THF $(100 \mathrm{~mL})$ was added $\mathrm{LiAlH}_{4}$ $(6.7 \mathrm{~g}, 176 \mathrm{mmol})$ slowly at ambient temperature. Stirred for one hour, the reaction was carefully quenched with saturated water $(6.7 \mathrm{~mL}), 10 \% \mathrm{NaOH}(13.4 \mathrm{~mL})$, and water $(20 \mathrm{~mL})$. Filtration and concentration of the filtrate provided the desired diol S1 (29 g) as white solid. The crude diol is pure enough for further transformation. mp $145-146{ }^{\circ} \mathrm{C} ;[\alpha]^{23} \mathrm{D}+42(c 0.45$, $\mathrm{CHCl}_{3}$ ); IR (KBr film) 3212, 2906, 1466, 1381, 1309, 833, $669 \mathrm{~cm}^{-1} ;{ }^{1} \mathrm{H}$ NMR $(300 \mathrm{MHz}$, $\left.\mathrm{CDCl}_{3}\right) \delta$ 5.57-5.62 (m, 2H, C2-H, C3-H), 4.36-4.43 (m, 1H, C16-H), 3.59-3.62 (m, 2H, C22$\mathrm{H}), 0.98\left(\mathrm{~d}, 3 \mathrm{H}, J=6.6 \mathrm{~Hz}, 21-\mathrm{CH}_{3}\right), 0.92\left(\mathrm{~s}, 3 \mathrm{H}, 18-\mathrm{CH}_{3}\right), 0.76\left(\mathrm{~s}, 3 \mathrm{H}, 19-\mathrm{CH}_{3}\right) ;{ }^{13} \mathrm{C} \mathrm{NMR}$ $\left(100 \mathrm{MHz}, \mathrm{CDCl}_{3}\right) \delta 125.9,125.8,72.5,70.3,62.0,54.3,54.1,42.5,41.4,40.3,39.7,35.8$, 35.2, 34.6, 32.6, 31.7, 30.2, 28.6, 20.6, 16.9, 13.2, 11.6; LRMS-ESI $(\mathrm{m} / \mathrm{z}): 355.0\left([\mathrm{M}+\mathrm{Na}]^{+}\right)$; HRMS-ESI $(\mathrm{m} / \mathrm{z}):[\mathrm{M}+\mathrm{Na}]^{+}$calcd for $\mathrm{C}_{22} \mathrm{H}_{36} \mathrm{O}_{2}$ 355.2607, found 355.2616.

\section{2-TBS-diol (8)}

Diol S1 (crude, $29 \mathrm{~g}$ ) was dissolved with dry DCM (200 $\mathrm{mL})$ and treated with imidazole (36 g, $529 \mathrm{mmol})$, DMAP (1.22 g, $10 \mathrm{mmol})$, and TBSCl $(26.7 \mathrm{~g}, 177 \mathrm{mmol})$ at ambient temperature. The reaction was stirred for 30 minutes, quenched with methanol, and diluted with $\mathrm{DCM} / \mathrm{H}_{2} \mathrm{O}(500 \mathrm{~mL} / 100 \mathrm{~mL})$. The organic layer was separated, washed with brine, dried over $\mathrm{Na}_{2} \mathrm{SO}_{4}$, and concentrated at reduced pressure. Flash column chromatography on silica gel provided 8 (38 g) as a colorless wax. The crude product is pure enough for the following step, the analytical sample was obtain by flash column chromatography on silica gel. $\mathrm{mp}$ 84-85 ${ }^{\circ} \mathrm{C} ;[\alpha]^{23} \mathrm{D}+14.8$ (c 1.30, $\mathrm{CHCl}_{3}$ ); IR (KBr film) 3417, 2929, 2854, 1470, 1254, 1062, 836, 776, $664 \mathrm{~cm}^{-1} ;{ }^{1} \mathrm{H}$ NMR $\left(300 \mathrm{MHz}, \mathrm{CDCl}_{3}\right) \delta$ 5.45-5.54 (m, 2H, C2-H, C3-H), 4.20-4.27 (m, 1H, C16-H), 3.83-3.87 (m, 1H), 3.36-3.48 (m, 2H, C22-H), 0.817 (s, 9H, TBS), 0 (s, 6H, TBS); ${ }^{13} \mathrm{C}$ NMR $\left(100 \mathrm{MHz}, \mathrm{CDCl}_{3}\right) \delta 125.8,125.8,76.7,72.2,63.2,54.3,54.1,42.9,41.4$, 40.4, 39.7, 35.2, 34.9, 34.6, 32.7, 31.7, 30.3, 28.7, 25.9, 20.6, 18.2, 16.8, 13.1, 11.7, -5.6, -5.7; LRMS-ESI $(\mathrm{m} / \mathrm{z}): 469.1\left([\mathrm{M}+\mathrm{Na}]^{+}\right)$; HRMS-ESI $(\mathrm{m} / \mathrm{z}):[\mathrm{M}+\mathrm{Na}]^{+}$calcd for $\mathrm{C}_{25} \mathrm{H}_{50} \mathrm{O}_{6}$ 469.3472 , found 469.3478 . 

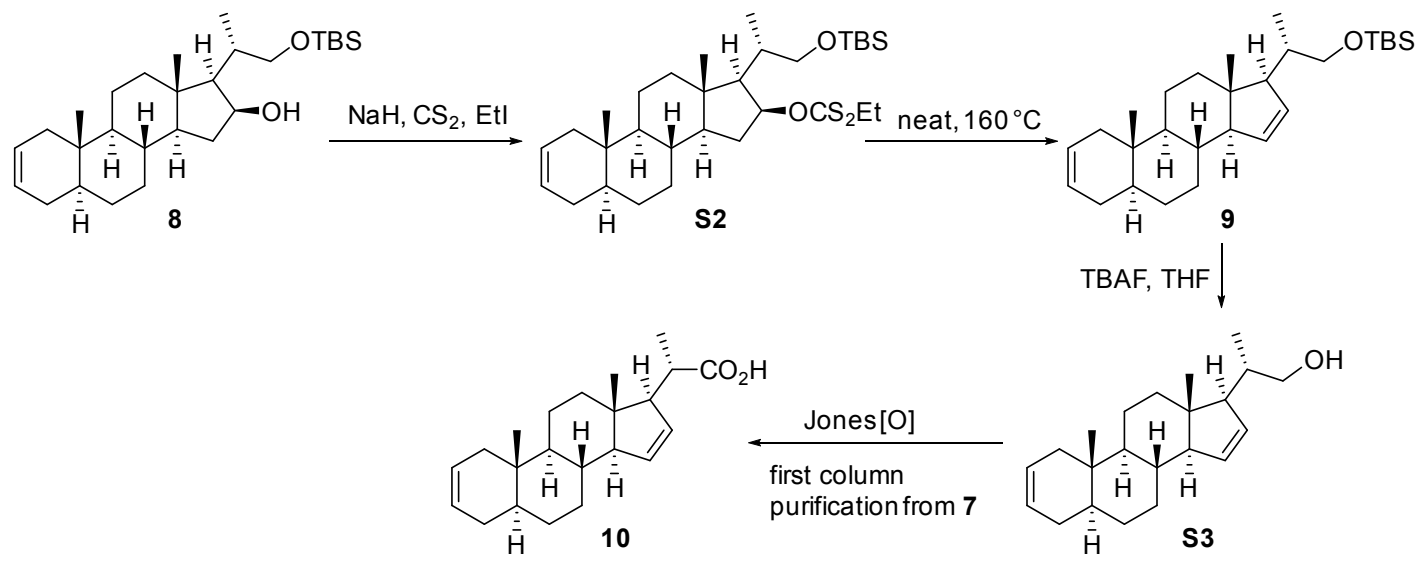

\section{Xanthate (S2)}

The crude 8 (38 g) was dissolved in anhydrous THF $(400 \mathrm{~mL})$, and to the solution was carefully added $\mathrm{NaH}(35 \mathrm{~g}, 60 \%, 852 \mathrm{mmol})$ at $0{ }^{\circ} \mathrm{C}$. One-hour stirring at ambient temperature gave a grey suspension, which was treated with $\mathrm{CS}_{2}(53 \mathrm{~mL}, 852 \mathrm{mmol})$, and with EtI (151 mL, $1.7 \mathrm{~mol})$ one hour later. The resulting red solution was stirred over night and quenched with water under ice-water bath until the mixture turned clear. The bilayer mixture was separated, extracted with EtOAc (200 mL*3), washed with brine, and dried over $\mathrm{Na}_{2} \mathrm{SO}_{4}$. Concentration under reduced pressure afforded red oil S2 (46 g, 99\%), which is used without purification. $[\alpha]^{23} \mathrm{D}+44.1\left(c\right.$ 0.05, $\left.\mathrm{CHCl}_{3}\right)$; IR ( $\mathrm{KBr}$ film) 2926, 2854, 1461, 1207, $1062,835,775,665 \mathrm{~cm}^{-1} ;{ }^{1} \mathrm{H}$ NMR $\left(300 \mathrm{MHz}, \mathrm{CDCl}_{3}\right) \delta$ 5.78-5.84 (m, 1H, C16-H), 5.56-5.60 (m, 2H, C2-H, C3-H), 3.25-3.46 (m, 2H, C22-H), 3.09 (q, $J=7.2 \mathrm{~Hz}, 2 \mathrm{H},-\mathrm{SCH}_{2} \mathrm{CH}_{3}$ ), 2.43-2.49 (m, 1H), 1.06 (d, 3H, $\left.J=6.9 \mathrm{~Hz}, 21-\mathrm{CH}_{3}\right), 0.86(\mathrm{~s}, 9 \mathrm{H}, \mathrm{TBS}), 0.75$ (s, 3H, 19- $\mathrm{CH}_{3}$ ); ${ }^{13} \mathrm{C}$ NMR $\left(100 \mathrm{MHz}, \mathrm{CDCl}_{3}\right) \delta 218.8,125.9,125.8,85.4,77.3,66.9,56.5,54.3,53.9,42.8$, 41.4, 39.6, 35.1, 34.6, 34.4, 33.1, 31.6, 30.2, 29.7, 29.7, 28.5, 25.9, 20.6, 18.3, 16.7, 13.4, 12.8, 11.6, -5.4; LRMS-ESI $(\mathrm{m} / \mathrm{z}): 573.0\left([\mathrm{M}+\mathrm{Na}]^{+}\right)$; HRMS-ESI $(\mathrm{m} / \mathrm{z}):[\mathrm{M}+\mathrm{Na}]^{+}$calcd for $\mathrm{C}_{21} \mathrm{H}_{54} \mathrm{O}_{2} \mathrm{~S}_{2} \mathrm{Si}$ 573.3226, found 573.3247.

\section{Chugaev elimination}

The flask containing S2 (46 g, crude product of previous step) was immersed in a oil bath and heated to $180{ }^{\circ} \mathrm{C}$ under reduced pressure provided by water bump. TLC indicated the complete consumption of $\mathbf{S 2}$ after ten hours. With the oil bath cooled to $80{ }^{\circ} \mathrm{C}$, to the flask were added THF $(400 \mathrm{~mL})$, TBAF $(70 \mathrm{~g}, 255 \mathrm{mmol})$, and HOAc $(50 \mathrm{~mL})$. The resulting solution was heated to refluxing for $12 \mathrm{~h}$ until TLC showed that the full consumption of 9 was realized. The solution was diluted with ethyl acetate $(1200 \mathrm{~mL})$ and water $(200 \mathrm{~mL})$. The organic layer was separated, washed with brine twice, and dried over $\mathrm{Na}_{2} \mathrm{SO}_{4}$. Filtration, concentration under reduced pressure afforded crude $\mathbf{S 3}$ as red wax, which was subjected to Jones oxidation without purification. Analytic sample was obtained via column chromatography on silica gel (PE/EA: 7/1) as white solid. Compound 9: $[\alpha]^{23} \mathrm{D}+4.0(c$ 5.95, $\mathrm{CHCl}_{3}$ ); IR (KBr film) 2927, 2855, 1462, 1257, 1086, 836, $776 \mathrm{~cm}^{-1} ;{ }^{1} \mathrm{H}$ NMR (300 MHz, $\left.\mathrm{CDCl}_{3}\right) \delta$ 5.73-5.81 (m, 2H, C15-H, C16-H), 5.55-5.62 (m, 2H, C2-H, C3-H), 3.72 (dd, 1H, $J$ 
$=12,4 \mathrm{~Hz}, \mathrm{C} 22-\mathrm{H}), 3.40-3.46(\mathrm{~m}, 1 \mathrm{H}, \mathrm{C} 22-\mathrm{H}), 0.95(\mathrm{~d}, 3 \mathrm{H}, J=6.3 \mathrm{~Hz}, \mathrm{C} 21-\mathrm{H}), 0.75(\mathrm{~s}, 3 \mathrm{H}$, $\left.19-\mathrm{CH}_{3}\right) ;{ }^{13} \mathrm{C} \mathrm{NMR}\left(100 \mathrm{MHz}, \mathrm{CDCl}_{3}\right) \delta 133.1,131.2,125.8,68.1,61.4,58.9,54.5,49.3$, 41.7, 39.6, 37.7, 35.4, 34.7, 32.8, 32.0, 30.4, 29.7, 28.6, 25.9, 25.6, 20.6, 18.3, 16.7, 12.8, 11.7, 1.1, -2.9, -3.0, -5.3, -5.3; LRMS-ESI $(m / z): 466.9\left([\mathrm{M}+\mathrm{K}]^{+}\right)$; HRMS-ESI $(m / z):[\mathrm{M}+\mathrm{Na}]$ ${ }^{+}$calcd for $\mathrm{C}_{28} \mathrm{H}_{48} \mathrm{OSi} 451.3367$, found 451.3360.

S3: $\mathrm{mp} 162-163{ }^{\circ} \mathrm{C} ;[\alpha]^{23} \mathrm{D}+38.79\left(c 1.50, \mathrm{CHCl}_{3}\right)$; IR ( $\mathrm{KBr}$ film) 3367, 2965, 1447, 1379, 1039, 966, $690 \mathrm{~cm}^{-1} ;{ }^{1} \mathrm{H}$ NMR (400 MHz, $\left.\mathrm{CDCl}_{3}\right) \delta 5.75-5.81(\mathrm{~m}, 2 \mathrm{H}, \mathrm{C} 15-\mathrm{H}, \mathrm{C} 16-\mathrm{H})$, 5.57-5.61 (m, 2H, C2-H, C3-H), 3.75-3.81 (m, 1H, C22-H), 3.50-3.56 (m, 1H, C22-H), 1.03 $(\mathrm{d}, 3 \mathrm{H}, J=6.8 \mathrm{~Hz}, \mathrm{C} 21-\mathrm{H}), 0.77\left(\mathrm{~s}, 3 \mathrm{H}, 19-\mathrm{CH}_{3}\right) ;{ }^{13} \mathrm{C} \mathrm{NMR}\left(100 \mathrm{MHz}, \mathrm{CDCl}_{3}\right) \delta 132.4$, 131.8, 125.9, 125.8, 68.0, 61.5, 58.6, 54.5, 49.4, 41.6, 39.6, 37.6, 35.2, 34.7, 32.7, 31.9, 30.3, 28.6, 20.6, 16.5, 12.8, 11.6; LRMS-ESI $(\mathrm{m} / \mathrm{z}): 315.3\left([\mathrm{M}+\mathrm{H}]^{+}\right)$; HRMS-ESI $(\mathrm{m} / \mathrm{z}):[\mathrm{M}+\mathrm{Na}]^{+}$ calcd for $\mathrm{C}_{22} \mathrm{H}_{34} \mathrm{O} 337.2501$, found 337.2503 .

\section{Jones oxidation of S3 to give 22-acid 10}

To a stirred solution of the crude alcohol $\mathbf{S 3}$ in acetone $(1000 \mathrm{~mL})$ at $0{ }^{\circ} \mathrm{C}$ was added Jones reagent $(2 \mathrm{~N})$ until the solution remained orange. The reaction was left to stir for $2 \mathrm{~h}$, and the resulting suspension was filtered and washed with acetone. The filtrate was concentrated in vacuo, diluted with ethyl acetate $(1000 \mathrm{~mL})$, THF $(200 \mathrm{~mL})$, and water $(200 \mathrm{~mL})$. The organic layer was separated, washed with brine twice, dried over $\mathrm{Na}_{2} \mathrm{SO}_{4}$, and concentrated under reduced pressure. Flash column chromatography on silica gel (PE/EA: 5/1) afforded 22-acid 10 (26.3 g, 91\% overall yield from 8) as a white solid. Known compound ${ }^{3}$ : ${ }^{1} \mathrm{H}$ NMR $\left(300 \mathrm{MHz}, \mathrm{CDCl}_{3}\right) \delta$ 5.85-5.93 (m, 1H, C16-H), 5.51-5.67 (m, 3H, C2-H, C3-H, C15-H), 2.53-2.66 (m, 1H, C20-H), 2.28-2.38 (m, 1H, C17-H), $1.25\left(\mathrm{~d}, 3 \mathrm{H}, J=6.6 \mathrm{~Hz}, 21-\mathrm{CH}_{3}\right), 0.77$ (s, 6H, 18- $\left.\mathrm{CH}_{3}, 19-\mathrm{CH}_{3}\right)$.

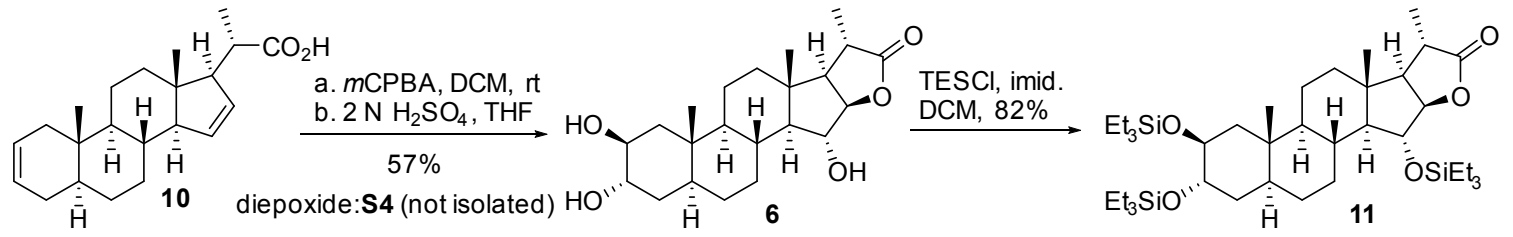

\section{Epoxidation and epoxide-opening reaction}

To a white suspension of diene-22-acid $10(580 \mathrm{~g}, 2.6 \mathrm{mmol})$ in 1,2-dichloroethane $(50 \mathrm{~mL})$ was added $m$ CPBA (3.34 g, $15.6 \mathrm{mmol})$. The resulting solution was left to stir for $12 \mathrm{~h}$ and TLC indicated the complete conversion of starting material. Quenched with saturated $\mathrm{Na}_{2} \mathrm{SO}_{3}$ aqueous solution, the reaction mixture was diluted with water. The organic layer was separated, washed with brine, dried over $\mathrm{Na}_{2} \mathrm{SO}_{4}$, and concentrated in vacuo to afford $\mathbf{S} 4$ as a white foam. Crude $\mathbf{S} 4$ was dissolved with THF (100 mL) and treated with $\mathrm{H}_{2} \mathrm{SO}_{4}(2 \mathrm{~N}, 5 \mathrm{~mL})$ at room temperature for $6 \mathrm{~h}$ until TLC showed the complete consumption of $\mathbf{S 4}$. The mixture

${ }^{3}$ Cong, R.; Zhang, Y.; Tian, W. Tetrahedron Lett. 2010, 51, (30), 3890-3892. 
was diluted with ethyl acetate, washed with brine, saturated $\mathrm{NaHSO}_{3}$ aqueous solution, and brine, dried over $\mathrm{Na}_{2} \mathrm{SO}_{4}$, and concentrated under reduced pressure. Flash column chromatography on silica gel (PE/EA: 1/1) gave 6 (530 mg, 6/3,4-epoxide isomer: 10/1, by ${ }^{1} \mathrm{H}$ NMR, 57\%) as a light blue gum and other byproducts (170 $\left.\mathrm{mg}, 20 \%\right)$. Known compound $^{3}$. IR (KBr film) 3419, 2933, 1755, 1456, 1037, $968 \mathrm{~cm}^{-1} ;{ }^{1} \mathrm{H}$ NMR (400 MHz, $\left.\mathrm{CD}_{3} \mathrm{OH}\right) \delta 4.79(\mathrm{dd}, 1 \mathrm{H}, \mathrm{C} 16-\mathrm{H}), 4.04(\mathrm{dd}, 1 \mathrm{H}, \mathrm{C} 16-\mathrm{H}), 3.76-3.85(\mathrm{~m}, 2 \mathrm{H}, \mathrm{C} 2-\mathrm{H}, \mathrm{C} 3-\mathrm{H})$, 2.66-2.78 (m, 1H, C20-H), $1.03\left(\mathrm{~s}, 3 \mathrm{H}, 18-\mathrm{CH}_{3}\right), 0.79\left(\mathrm{~s}, 3 \mathrm{H}, 19-\mathrm{CH}_{3}\right)$; LRMS-ESI $(\mathrm{m} / \mathrm{z}): 423$ $\left(\left[\mathrm{M}+\mathrm{HCO}_{2}\right]^{-}\right)$; HRMS-ESI $(\mathrm{m} / \mathrm{z})$ : [M-H $]^{-}$calcd for $\mathrm{C}_{22} \mathrm{H}_{34} \mathrm{O}_{5}$ 377.2333, found 377.2343.

\section{Compound 11}

To a light yellow solution of triol $6(0.93 \mathrm{~g}, 2.46 \mathrm{mmol})$ and imidazole $(1.7 \mathrm{~g}, 24.6 \mathrm{mmol})$ was slowly added TESCl $(4.6 \mathrm{~mL}, 24.6 \mathrm{mmol})$. The reaction was left to stir at room temperature for one hour and quenched with saturated $\mathrm{NaHCO}_{3}$ aqueous solution. The reaction mixture was extracted with DCM; the combined organic layer was dried over $\mathrm{Na}_{2} \mathrm{SO}_{4}$, filtered, and concentrated under reduced pressure. Flash column chromatography on silica gel (PE/EA: 80/1) afforded $11(1.43 \mathrm{~g}, 82 \%)$ as a colorless oil. [ $\alpha]^{25} \mathrm{D}-9.89$ (c 4.90, $\mathrm{CHCl}_{3}$ ); IR (KBr film) 2952, 2876, 1779, 1456, 1077, 1011, $742 \mathrm{~cm}^{-1} ;{ }^{1} \mathrm{H}$ NMR (300 MHz, $\left.\mathrm{CDCl}_{3}\right) \delta 4.56(\mathrm{dd}, 1 \mathrm{H}, J=8.1,2.4 \mathrm{~Hz}, \mathrm{C} 16-\mathrm{H}), 4.06(\mathrm{dd}, 1 \mathrm{H}, J=8.1,2.1 \mathrm{~Hz}, \mathrm{C} 15-\mathrm{H})$, 3.62-3.67 (m, 2H, C2-H, C3-H), 2.56 (q, 1H, $J=7.8 \mathrm{~Hz}, \mathrm{C} 20-\mathrm{H}), 2.07$ (d, $1 \mathrm{H}, J=7.8 \mathrm{~Hz}$, $\mathrm{C} 17-\mathrm{H}) ;{ }^{13} \mathrm{C} \mathrm{NMR}\left(100 \mathrm{MHz}, \mathrm{CDCl}_{3}\right) \delta 181.1,91.7,80.4,72.2,71.3,59.5,56.5,55.1,41.3$, 40.2, 39.0, 38.6, 36.1, 36.0, 34.6, 32.1, 32.0, 28.0, 20.0, 17.8, 15.2, 14.3, 6.9, 6.8, 6.7, 6.4, 4.9, 4.8, 4.7; LRMS-ESI $(\mathrm{m} / \mathrm{z}): 721.5\left([\mathrm{M}+\mathrm{H}]^{+}\right)$; HRMS-ESI $(\mathrm{m} / \mathrm{z}):[\mathrm{M}+\mathrm{Na}]^{+}$calcd for $\mathrm{C}_{40} \mathrm{H}_{76} \mathrm{O}_{5} \mathrm{Si}_{3} 743.4892$, found 743.4869 .

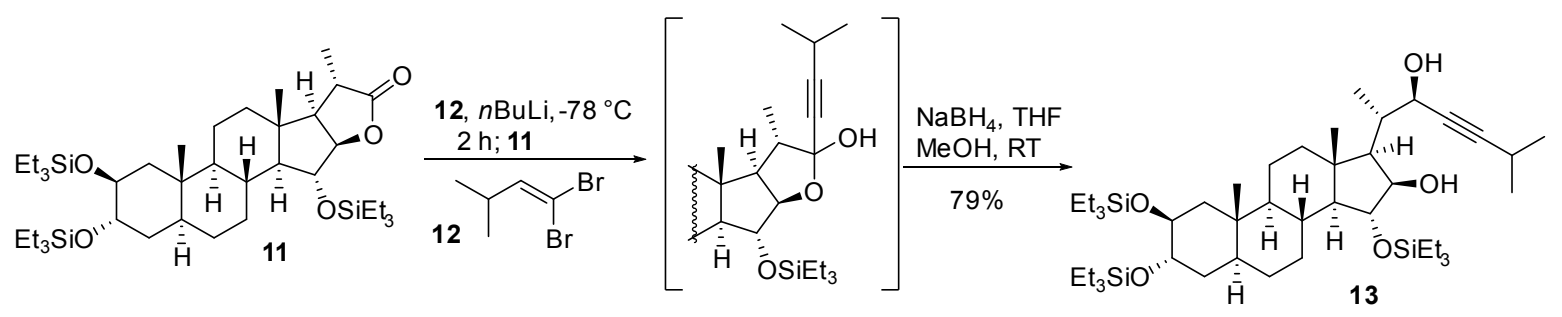

\section{Diol 13}

To a solution of 1,1-dibromo-3-methylbut-1-ene $(\mathbf{1 2}, 0.336 \mathrm{~g}, 1.47 \mathrm{mmol})$ in anhydrous THF $(10 \mathrm{~mL})$ was added $n$-BuLi (1.6 M, $1.84 \mathrm{~mL}, 2.94 \mathrm{mmol})$ at $-78{ }^{\circ} \mathrm{C}$ under Ar atmosphere. To the solution was slowly added a solution of lactone $12(0.70 \mathrm{~g}, 0.97 \mathrm{mmol})$ in anhydrous THF $(10 \mathrm{~mL})$ after two-hour stirring. The reaction mixture was allowed to stir at ambient temperature for $12 \mathrm{~h}$ before quenched with saturated $\mathrm{NH}_{4} \mathrm{Cl}$ aqueous solution. The organic solvent was removed under reduced pressure and ethyl acetate was added to dissolve the residue. The resultant solution was washed with water, dried over $\mathrm{Na}_{2} \mathrm{SO}_{4}$, filtered, and concentrated in vacuo to afford a colorless oil. The crude adduct was dissolved with $\mathrm{MeOH} /$ 
THF $(20 \mathrm{~mL} / 20 \mathrm{~mL})$ to give a colorless solution to which was added $\mathrm{NaBH}_{4}(98 \mathrm{mg}, 2.91$ $\mathrm{mmol}$ ) at $0{ }^{\circ} \mathrm{C}$. The reaction was allowed to warm to ambient temperature and to stir overnight. Quenched with water, concentrated under reduced pressure to remove to organic solvents, the reaction mixture was dissolved with ethyl acetate, washed with water, dried over $\mathrm{Na}_{2} \mathrm{SO}_{4}$, filtered, and concentrated to dryness. Flash column chromatography on silica gel (PE/EA: 40/1) afforded diol $13(0.61 \mathrm{~g}, 79 \%)$ as a colorless oil. $[\alpha]^{25} \mathrm{D}+44.2\left(c 1.20, \mathrm{CHCl}_{3}\right)$; IR (KBr film) 2952, 2876, 1456, 1238, 1075, 1009, 823, $744 \mathrm{~cm}^{-1} ;{ }^{1} \mathrm{H}$ NMR (400 MHz, $\left.\mathrm{CDCl}_{3}\right) \delta 4.39$ (s like, 1H, C22-H), $4.07(\mathrm{~m}, 1 \mathrm{H}, \mathrm{C} 16-\mathrm{H}), 3.86(\mathrm{~d}, 1 \mathrm{H}, J=10.4 \mathrm{~Hz}, \mathrm{C} 15-\mathrm{H})$, 3.63-3.69 (m, 2H, C2-H, C3-H), 2.58 (dq, $J=6.8,10.0$ Hz, 1H, C26-H), 1.18 (d, 6H, $J=7.2$ $\left.\mathrm{Hz}, 26-\mathrm{CH}_{3}, 27-\mathrm{CH}_{3}\right) ;{ }^{13} \mathrm{C} \mathrm{NMR}\left(100 \mathrm{MHz}, \mathrm{CDCl}_{3}\right) \delta$ 93.2, 83.4, 81.7, 77.7, 72.3, 71.4, 69.6, $60.3,54.9,54.7,43.3,40.7,40.2,38.5,35.9,34.9,34.5,32.3,32.0,28.2,23.0,20.5,16.9$, 14.8, 14.3, 7.0, 6.9, 6.8, 5.0, 4.9, 4.8; LRMS-ESI $(\mathrm{m} / \mathrm{z}): 813.6\left([\mathrm{M}+\mathrm{Na}]^{+}\right)$; HRMS-MALDI $(\mathrm{m} / \mathrm{z}):[\mathrm{M}+\mathrm{Na}]^{+}$calcd for $\mathrm{C}_{45} \mathrm{H}_{86} \mathrm{O}_{5} \mathrm{Si}_{3} 813.5675$, found 813.5671 .

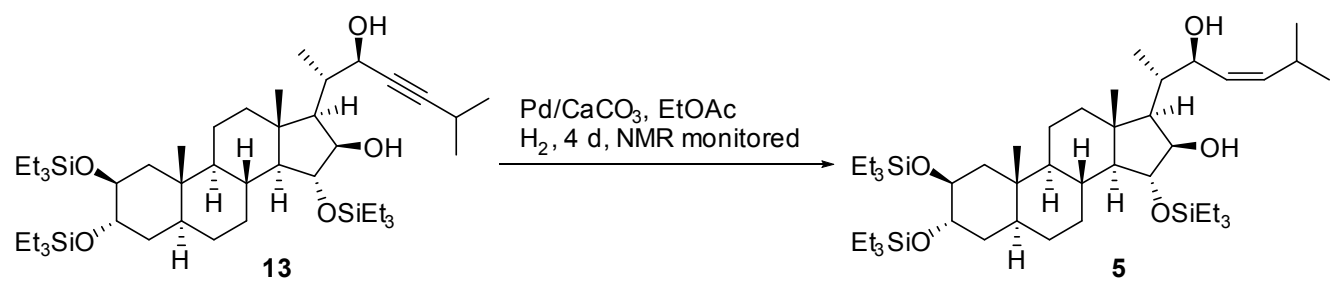

\section{Rosenmond reduction (5)}

A suspension of $\mathrm{Pd} / \mathrm{CaCO}_{3}(5 \%, 350 \mathrm{mg})$ and the alkyne $13(500 \mathrm{mg}, 0.63 \mathrm{mmol})$ in EtOAc $(20 \mathrm{~mL})$ was stirred for $30 \mathrm{~min}$. The flask was charged with hydrogen $(1 \mathrm{~atm})$, and the reaction mixture was stirred until ${ }^{1} \mathrm{H}$ NMR analysis indicated complete conversion (4 days). The reaction mixture was filtered through Celite and concentrated in vacuo. The crude product was purified by flash column chromatography on silica gel (PE/EA: 40/1) to give 5 (492 mg, 98\%) as a colorless oil. $[\alpha]^{25} \mathrm{D}+16.9$ (c 0.75, $\left.\mathrm{CHCl}_{3}\right)$; IR ( $\mathrm{KBr}$ film) 3415, 2955, 2876, 1458, 1376, 1239, 1077, 1006, 978, $745 \mathrm{~cm}^{-1}$; ${ }^{1} \mathrm{H}$ NMR (300 MHz, $\left.\mathrm{CDCl}_{3}\right) \delta$ 5.36-5.42 (m, 2H, C22-H, C23-H), 4.48 (d, 1H, J=10.2 Hz, C22-H), 4.13 (s, 1H), 4.03-4.09 (d, 1H, $J=$ 7.2 Hz, C16-H), 3.85 (d, 1H, $J=10.2 \mathrm{~Hz}, \mathrm{C} 15-\mathrm{H}), 3.64-3.68$ (m, 2H, C2-H, C3-H), 2.25-2.39 $(\mathrm{m}, 2 \mathrm{H}, \mathrm{C} 17-\mathrm{H}, \mathrm{C} 20-\mathrm{H}) ;{ }^{13} \mathrm{C} \mathrm{NMR}\left(100 \mathrm{MHz}, \mathrm{CDCl}_{3}\right) \delta 141.9,125.1,83.3,81.8,73.8,72.3$, 71.4, 60.4, 55.0, 53.9, 43.6, 40.7, 40.2, 38.5, 35.8, 34.5, 34.4, 32.3, 32.0, 28.2, 27.0, 23.4, 23.0, 20.5, 16.9, 14.5, 14.3, 7.0, 6.9, 6.8, 5.0, 4.9, 4.8; LRMS-ESI $(m / z): 775.5\left(\left[\mathrm{M}-\mathrm{H}_{2} \mathrm{O}+\mathrm{H}\right]\right.$ $\left.{ }^{+}\right)$; HRMS-ESI $(\mathrm{m} / z)$ : $[\mathrm{M}+\mathrm{Na}]^{+}$calcd for $\mathrm{C}_{45} \mathrm{H}_{88} \mathrm{O}_{5} \mathrm{Si}_{3} 815.5831$, found 815.5791.
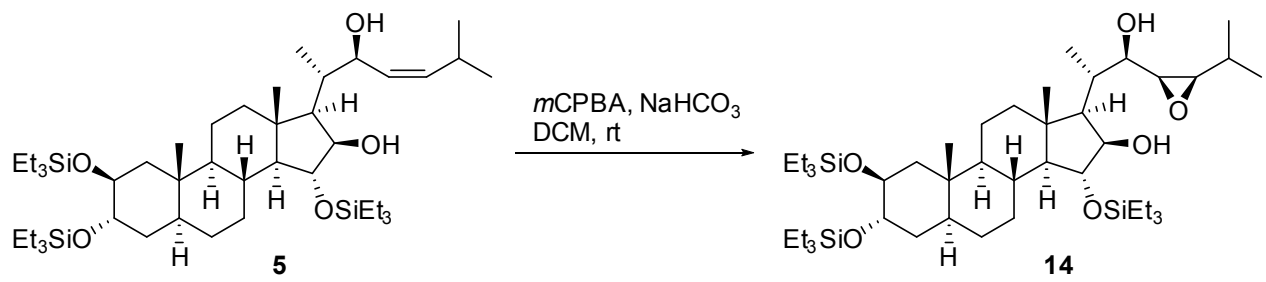


\section{Epoxide 14}

To a solution of cis-alkene $5(390 \mathrm{mg}, 0.49 \mathrm{mmol})$ in DCM $(10 \mathrm{~mL})$ were added $\mathrm{NaHCO}_{3}$ (146 mg, $4.9 \mathrm{mmol})$ and $m \mathrm{CPBA}(85 \%, 153 \mathrm{mg}, 0.98 \mathrm{mmol}) .{ }^{4}$ The reaction mixture was left to stir at ambient temperature for three hours, quenched with saturated $\mathrm{Na}_{2} \mathrm{SO}_{3}$ aqueous solution, and extracted with DCM $(20 \mathrm{~mL})$. The combined organic layer was washed with brine, dried over $\mathrm{Na}_{2} \mathrm{SO}_{4}$, and concentrated in vacuo. The crude product was purified by flash column chromatography on silica gel (PE/EA: 30/1) to furnish epoxide 14 (375 mg, 95\%) as a colorless oil. $[\alpha]^{25} \mathrm{D}+19.4\left(c\right.$ 0.90, $\left.\mathrm{CHCl}_{3}\right)$; IR (KBr film) 3418, 2954, 2876, 1456, 1377, 1237, 1092, 1046, 828, $745 \mathrm{~cm}^{-1} ;{ }^{1} \mathrm{H}$ NMR $\left(300 \mathrm{MHz}, \mathrm{CDCl}_{3}\right) \delta 4.04(\mathrm{~d}, 1 \mathrm{H}, J=6.0 \mathrm{~Hz}$, C16-H), 3.84 (dd, 1H, J=9.6, 2.4 Hz, C15-H), 3.64-3.72 (m, 3H, C22-H, C2-H, C3-H), 3.20 $(\mathrm{dd}, 1 \mathrm{H}, J=4.0,6.0 \mathrm{~Hz}, \mathrm{C} 23-\mathrm{H}), 2.77(\mathrm{dd}, 1 \mathrm{H}, J=4.0,9.6 \mathrm{~Hz}, \mathrm{C} 24-\mathrm{H}) ;{ }^{13} \mathrm{C}$ NMR $(100 \mathrm{MHz}$, $\left.\mathrm{CDCl}_{3}\right) \delta 83.8,81.7,72.3,72.0,71.4,64.5,60.4,58.2,55.0,54.2,43.54,40.6,40.2,38.6$, $35.9,34.5,33.5,32.3,32.0,28.2,26.7,20.5,20.2,18.6,15.2,14.6,14.3,6.9,6.9,6.8,5.0$, 4.9, 4.8. MS is unable to give the molecular weight of 14.

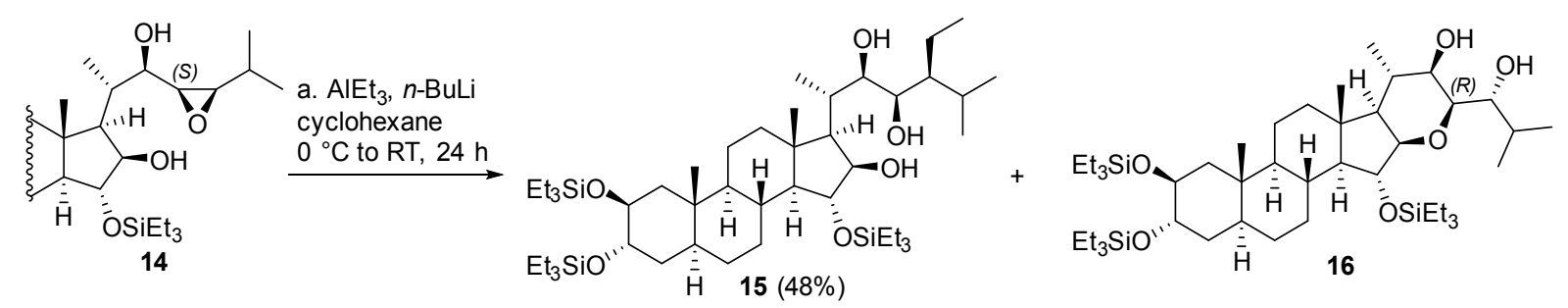

\section{Epoxide opening ${ }^{5}$ products 15 and 16}

To a triethylaluminum solution $(0.60 \mathrm{M}$ in hexane, purchased from Aldrich, $2.0 \mathrm{~mL})$ was added $n \operatorname{BuLi}(1.6 \mathrm{M}$ in hexane, $0.20 \mathrm{~mL})$ at $0{ }^{\circ} \mathrm{C}$. To the resultant light blue solution was added a solution of epoxide $14(28 \mathrm{mg}, 0.03 \mathrm{mmol})$ in cyclohexane $(10 \mathrm{~mL})$. The reaction mixture was allowed to warm to ambient temperature and stir for $24 \mathrm{~h}$ until TLC indicated that the full consumption of $\mathbf{1 4}$ was achieved. Quenched with water, the reaction system was filtered through Celite, diluted with ethyl acetate, washed with brine, and dried over $\mathrm{Na}_{2} \mathrm{SO}_{4}$. The crude product was purified by flash column chromatography on silica gel (PE/EA: 25/1, then 10/1) to furnish desired 15 (14 mg, 48\%, less polar) as a colorless oil and byproduct 16 (10 mg, 36\%) as a colorless oil. Compound 15: $[\alpha]^{25} \mathrm{D}+5.08\left(c \mathrm{0.25}, \mathrm{CHCl}_{3}\right)$; IR ( $\mathrm{KBr}$ film) 2960, 2926, 1729, 1462, 1260, 1092, 1018, $800 \mathrm{~cm}^{-1}$; ${ }^{1} \mathrm{H}$ NMR (400 MHz, $\left.\mathrm{CDCl}_{3}\right) \delta 4.05(\mathrm{~d}$, $1 \mathrm{H}, J=7.2 \mathrm{~Hz}, \mathrm{C} 16-\mathrm{H}), 3.85$ (d, 1H, $J=9.6 \mathrm{~Hz}, \mathrm{C} 15-\mathrm{H}), 3.68-3.86$ (m, 4H, C2-H, C3-H, C22-H, C23-H), 2.21 (m, 1H, C20-H); $\left.{ }^{13} \mathrm{C} \mathrm{NMR} \mathrm{(100} \mathrm{MHz,} \mathrm{CDCl}_{3}\right) \delta 84.3,82.1,75.5,73.0$, $72.3,71.4,60.4,55.0,54.7,40.4,40.2,39.3,38.5,37.1,34.5,32.8,32.2,32.0,28.2,27.5$, $20.8,20.4,15.6,14.8,14.7,14.3,13.4,11.7,9.4,7.0,6.9,6.8,5.1,4.9,4.8$; LRMS-ESI $(\mathrm{m} / \mathrm{z})$ :

\footnotetext{
4 Back, T. G.; Baron, D. L. Can. J. Chem. 1996, 74, (10), 1857-1867.

${ }^{5}$ Mori, K.; Sakakibara, M.; Okada, K. Tetrahedron 1984, 40, (10), 1767-1781.
} 
$861.8\left([\mathrm{M}+\mathrm{Na}]^{+}\right)$; HRMS-ESI $(\mathrm{m} / \mathrm{z}):[\mathrm{M}+\mathrm{Na}]^{+}$calcd for $\mathrm{C}_{47} \mathrm{H}_{94} \mathrm{O}_{6} \mathrm{Si}_{3}$ 861.6250, found 861.6241. Compound 16: ${ }^{1} \mathrm{H}$ NMR (400 MHz, $\left.\mathrm{CDCl}_{3}\right) \delta 3.93(\mathrm{~d}, J=6.2 \mathrm{~Hz}, 1 \mathrm{H}, \mathrm{C16}-\mathrm{H})$, 3.81-3.83 (m, 2H, C15-H, C23-H), 3.65-3.68 (m, 2H, C2-H, C3-H), 3.56-3.60 (m, 1H, C22$\mathrm{H}), 3.26(\mathrm{~m}, 1 \mathrm{H}, \mathrm{C} 23-\mathrm{H}) ;{ }^{13} \mathrm{C} \mathrm{NMR}\left(100 \mathrm{MHz}, \mathrm{CDCl}_{3}\right) \delta 83.9,82.0,78.7,76.7,72.3,71.4$, 60.2 , 56.5, 55.0, 43.5, 40.7, 40.2, 38.6, 36.4, 35.8, 34.5, 34.2, 32.3, 32.0, 30.3, 29.6, 28.1, 20.5, 18.3, 17.3, 14.6, 14.3, 13.6, 6.9, 6.9, 6.8, 5.0, 4.9, 4.8; LRMS-ESI $(\mathrm{m} / \mathrm{z}): 831.2([\mathrm{M}+\mathrm{Na}]$ ${ }^{+}$); HRMS-ESI $(\mathrm{m} / z)$ : $[\mathrm{M}+\mathrm{Na}]^{+}$calcd for $\mathrm{C}_{45} \mathrm{H}_{88} \mathrm{O}_{6} \mathrm{Si}_{3} 831.5781$, found 831.5776.
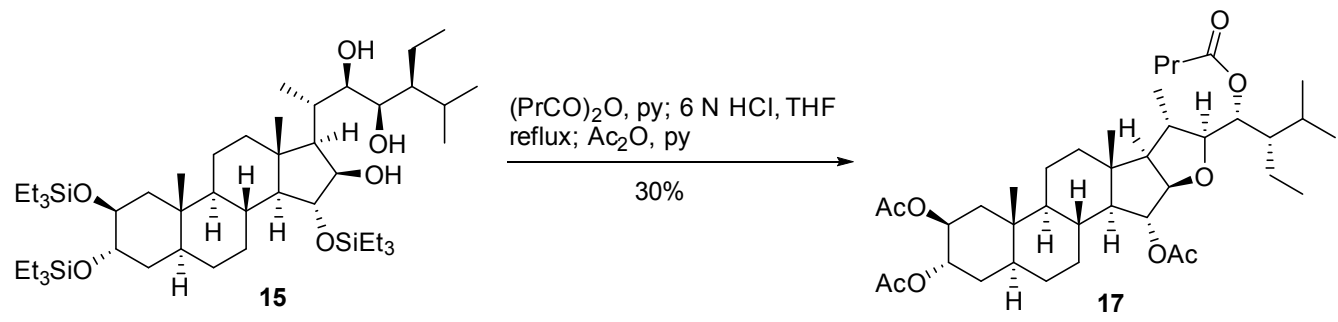

\section{Compound 17}

Added butyric anhydride ( $1 \mathrm{~mL})$, a solution of triol $15(120 \mathrm{mg}, 0.14 \mathrm{mmol})$ and DMAP (1 $\mathrm{mg})$ in dry pyridine $(2 \mathrm{~mL})$ was stirred at ambient temperature for $18 \mathrm{~h}$ before the solvent was removed in vacuo. The residue was dissolved in THF $(2.0 \mathrm{~mL})$ and to the solution was added concentrated $\mathrm{HCl}(1 \mathrm{~mL})$. The reaction mixture was heated to reflux for $18 \mathrm{~h}$, cooled to room temperature, diluted with ethyl acetate $(40 \mathrm{~mL})$, washed with brine, dried over $\mathrm{Na}_{2} \mathrm{SO}_{4}$, filtered, and concentrated in vacuo. The crude product was dissolve with pyridine $(2 \mathrm{~mL})$ and $\mathrm{Ac}_{2} \mathrm{O}(1.0 \mathrm{~mL})$. The resulting solution was stirred at room temperature for $20 \mathrm{~h}$ and the solvent was removed under high vacuo. The crude product was purified by flash column chromatography on silica gel (PE/EA: 8/1) to give 17 (36 mg, 30\%) as a colorless oil. NOESY crosspeaks of $\mathrm{C} 22-\mathrm{H}$ and $\mathrm{C} 16-\mathrm{H} / \mathrm{C} 21-\mathrm{Me}$ proved $\mathrm{C22}$ is $\boldsymbol{S}$-configured. IR ( $\mathrm{KBr}$ film) 2961, 2933, 1740, 1368, 1235, 1034, $969 \mathrm{~cm}^{-1} ;{ }^{1} \mathrm{H}$ NMR (400 MHz, $\left.\mathrm{CDCl}_{3}\right) \delta 5.21$ (dd, $1 \mathrm{H}, J=7.6,2.8 \mathrm{~Hz}, \mathrm{C} 23-\mathrm{H}), 5.11$ (dd, 1H, $J=10.4,2.0 \mathrm{~Hz}, \mathrm{C} 15-\mathrm{H}), 4.87-4.90$ (m, 2H, C2H, C3-H), 4.14 (dd, 1H, J =7.6, 2.0 Hz, C15-H), 3.54 (t, 1H, J=7.6 Hz, C22-H), 2.27 (t, 2H, $J=6.8 \mathrm{~Hz}), 2.00-2.01\left(\mathrm{~s}, 9 \mathrm{H}\right.$, three OAc), $2.21(\mathrm{~m}, 1 \mathrm{H}, \mathrm{C} 20-\mathrm{H}) ;{ }^{13} \mathrm{C} \mathrm{NMR}(100 \mathrm{MHz}$, $\left.\mathrm{CDCl}_{3}\right) \delta 173.1,170.3,169.7,90.0,87.9,80.0,75.6,70.1,69.7,63.6,59.5,54.6,42.1,39.3$, 37.7, 37.6, 36.8, 36.4, 35.4, 34.9, 34.4, 30.8, 29.6, 29.1, 27.6, 27.1, 21.4, 21.2, 21.2, 21.0, $20.2,18.3,17.0,15.6,13.7,13.7,11.4,10.0$; LRMS-ESI $(\mathrm{m} / \mathrm{z}): 697.5\left([\mathrm{M}+\mathrm{Na}]^{+}\right)$; HRMSMALDI $(m / z):[\mathrm{M}+\mathrm{Na}]^{+}$calcd for $\mathrm{C}_{39} \mathrm{H}_{62} \mathrm{NaO}_{9} 697.4286$, found 697.4306 . 
Table 1. Comparing 1H NMR Data of 17 with 2

\begin{tabular}{|c|c|c|c|c|c|}
\hline No. & Reported 2 & Synthetic 17 & No. & Reported 2 & Synthetic 17 \\
\hline 2 & $4.90 \mathrm{bs}$ & $4.90 \mathrm{bs}$ & 22 & $3.52 \mathrm{dd}(8.7,6.9)$ & $3.54 \mathrm{t}(7.6)$ \\
\hline 3 & $4.87 \mathrm{bs}$ & $4.87 \mathrm{bs}$ & 23 & $5.27 \mathrm{dd}(8.7,2.7)$ & $5.21 \mathrm{dd}(7.6,2.8)$ \\
\hline 15 & $5.11 \mathrm{dd}(10.6,2.1)$ & $5.11 \mathrm{dd}(10.4,2.0)$ & $2^{\prime}$ & $\begin{array}{c}2.28 \mathrm{dt}(2.5,6.5 \\
2 \mathrm{H})\end{array}$ & $2.27 \mathrm{t}(6.8,2 \mathrm{H})$ \\
\hline 16 & $4.14 \mathrm{dd}(7.7,2.1)$ & $4.14 \mathrm{dd}(7.6,2.0)$ & $3^{\prime}$ & $1.66(2 \mathrm{H})$ & $1.6-1.7(2 \mathrm{H})$ \\
\hline 18 & $0.87 \mathrm{~s}$ & $0.87 \mathrm{~s}$ & $2 \mathrm{Ac}$ & $2.01 \mathrm{~s}$ & $2.00-2.01$ \\
\hline 19 & $0.89 \mathrm{~s}$ & $0.87-0.90$ & $3 \mathrm{Ac}$ & $2.02 \mathrm{~s}$ & $2.00-2.01$ \\
\hline 20 & 2.03 & $2.00-2.01$ & & & \\
\hline
\end{tabular}

Table 2. Comparing 13C NMR Data of 17 with 2

\begin{tabular}{|c|c|c|c|c|c|}
\hline No. & Reported 2 & Synthetic 17 & No. & Reported 2 & Synthetic 17 \\
\hline 1 & 37.7 & 37.7 & 19 & 12.8 & 11.4 \\
\hline 2 & 70.1 & 70.1 & 20 & 35.8 & 35.0 \\
\hline 3 & 69.8 & 69.8 & 21 & 18.6 & 21.1 \\
\hline 4 & 29.7 & 29.7 & 22 & 87.2 & 88.0 \\
\hline 5 & 39.2 & 39.3 & 23 & 75.4 & 75.6 \\
\hline 6 & 30.9 & 30.9 & 24 & 46.3 & 37.8 \\
\hline 7 & 27.6 & 27.6 & 25 & 26.5 & 36.8 \\
\hline 8 & 34.5 & 34.4 & 26 & 22.9 & 15.7 \\
\hline 9 & 54.6 & 54.6 & 27 & 18.0 & 13.7 \\
\hline 10 & 35.4 & 35.4 & 28 & 18.6 & 27.2 \\
\hline 11 & 20.2 & 20.2 & 29 & 13.8 & 10.0 \\
\hline 12 & 39.3 & 39.3 & $1^{\prime}$ & 170.1 & 173.1 \\
\hline 13 & 42.1 & 42.1 & $2^{\prime}$ & 36.4 & 36.5 \\
\hline 14 & 59.7 & 59.5 & $3^{\prime}$ & 18.3 & 18.3 \\
\hline 15 & 80.1 & 80.1 & $4^{\prime}$ & 13.9 & 13.8 \\
\hline 16 & 90.2 & 90.2 & $2 \mathrm{AC}$ & $168.4,21.5$ & $170.3,21.5$ \\
\hline 17 & 63.7 & 63.7 & $3 \mathrm{AC}$ & $168.3,21.3$ & $169.7,21.3$ \\
\hline 18 & 17.0 & 13.7 & $15 \mathrm{Ac}$ & $168.3,21.3$ & $169.7,21.2$ \\
\hline
\end{tabular}




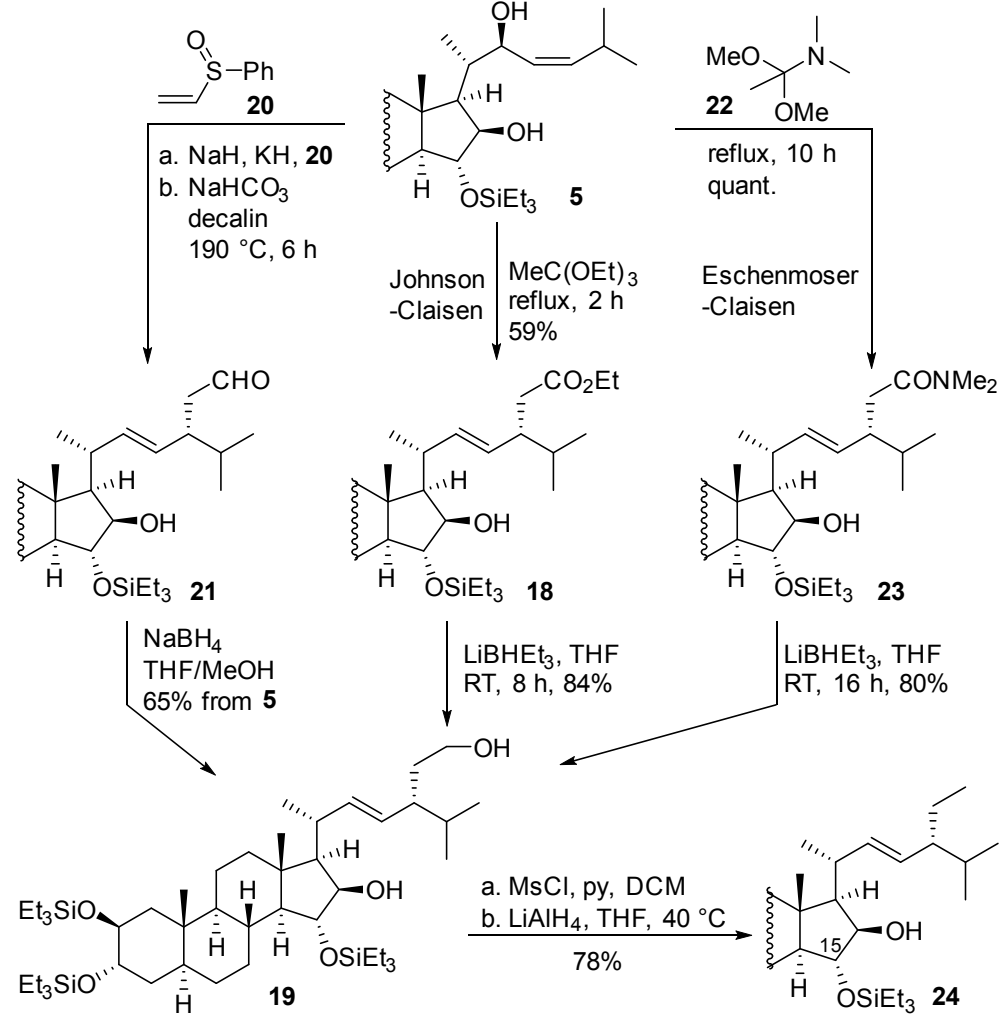

\section{Johnson-Claisen rearrangement product 18}

A flask containing allylic alcohol $5(120 \mathrm{mg}, 0.15 \mathrm{mmol})$ and triethyl orthoacetate was heated to reflux for two hours. The solvent was removed under reduced pressure. The crude product was subjected to flash column chromatography on silica gel (PE/EA: 100/1) to afford 18 (79 $\mathrm{mg}, 59 \%)$ as a colorless oil. $[\alpha]^{25} \mathrm{D}+6.92\left(c\right.$ 2.00, $\left.\mathrm{CHCl}_{3}\right)$; IR ( $\mathrm{KBr}$ film) 3552, 2954, 2876, 1730, 1458, 1371, 1260, 1239, 1124, 1016, $745 \mathrm{~cm}^{-1} ;{ }^{1} \mathrm{H}$ NMR $\left(400 \mathrm{MHz}, \mathrm{CDCl}_{3}\right) \delta$ 5.22-5.34 (m, 2H, C22-H, C23-H), 3.99-4.05 (q, 2H, $J=6.8 \mathrm{~Hz}), 3.75-3.77$ (m, 2H, C15-H, C16-H), 3.58-3.62 (m, 2H, C2-H, C3-H), 2.65 (m, 1H), 2.14-2.44 (m, 3H); ${ }^{13} \mathrm{C}$ NMR (100 $\left.\mathrm{MHz}, \mathrm{CDCl}_{3}\right) \delta 173.3,139.3,129.5,83.9,81.6,72.3,71.4,60.3,59.1,58.2,55.0,45.6,42.7$, $40.8,40.2,38.5,37.9,35.9,35.2,34.5,32.3,32.1,31.9,28.2,21.6,20.5,20.4,18.9,14.8$, $14.3,14.2,7.0,6.9,6.8,5.0,4.9,4.8$; LRMS-MALDI $(\mathrm{m} / \mathrm{z}): 885.6\left([\mathrm{M}+\mathrm{Na}]^{+}\right)$; HRMSMALDI $(\mathrm{m} / \mathrm{z}):[\mathrm{M}+\mathrm{Na}]^{+}$calcd for $\mathrm{C}_{49} \mathrm{H}_{94} \mathrm{O}_{6} \mathrm{Si}_{3}$ 885.6250, found 885.6237.

\section{Reduction of 18 to 19}

To a solution of 18 (500 $\mathrm{mg}, 0.58 \mathrm{mmol})$ in anhydrous THF (5 mL) was slowly added $\mathrm{LiBHEt}_{3}(1.0 \mathrm{M}, 5.0 \mathrm{~mL})$ at $0{ }^{\circ} \mathrm{C}$. The reaction system was left to stir at ambient temperature for eight hours before saturated $\mathrm{NH}_{4} \mathrm{Cl}$ aqueous solution was added to quench to reaction. The mixture was diluted with ethyl acetate, separated, and dried the organic layer over $\mathrm{Na}_{2} \mathrm{SO}_{4}$. The solvent was removed under reduced pressure. The crude product was subjected to flash column chromatography on silica gel (PE/EA: 20/1) to afford 19 (411 $\mathrm{mg}, 84 \%)$ as a colorless oil. $[\alpha]^{25} \mathrm{D}+29.4\left(c\right.$ 0.15, $\left.\mathrm{CHCl}_{3}\right)$; IR ( $\mathrm{KBr}$ film) 3403, 2954, 2876, 1458, 1371, 1240, 1092, 1069, 1015, $745 \mathrm{~cm}^{-1} ;{ }^{1} \mathrm{H}$ NMR (400 MHz, $\left.\mathrm{CDCl}_{3}\right) \delta$ 5.22-5.34 (m, 2H, C22-H, 
C23-H), 3.75-3.77 (m, 2H, C15-H, C16-H), 3.58-3.62 (m, 4H, C2-H, C3-H, C29-H), 2.49 (m, $1 \mathrm{H}, \mathrm{C} 24-\mathrm{H}) ;{ }^{13} \mathrm{C} \mathrm{NMR}\left(100 \mathrm{MHz}, \mathrm{CDCl}_{3}\right) \delta 138.7,130.5,83.5,82.0,72.3,61.8,59.6,58.2$, 55.0, 46.8, 42.8, 40.7, 38.6, 35.9, 35.4, 34.9, 34.5, 32.3, 32.1, 29.6, 28.2, 22.6, 21.3, 20.6, 20.5, 18.9, 14.8, 14.3, 7.0, 6.9, 6.8, 5.0, 4.9, 4.8; LRMS-MALDI $(m / z): 843.6\left([\mathrm{M}+\mathrm{Na}]^{+}\right)$; HRMS-MALDI $(\mathrm{m} / \mathrm{z}):[\mathrm{M}+\mathrm{Na}]^{+}$calcd for $\mathrm{C}_{47} \mathrm{H}_{92} \mathrm{O}_{5} \mathrm{Si}_{3} 843.6144$, found 843.6147 .

\section{Eschenmoser-Claisen rearrangement product 23}

A flask containing allylic alcohol $5(40 \mathrm{mg}, 0.05 \mathrm{mmol})$ and $22(5 \mathrm{~mL})$ was heated to reflux for 10 hours. The solvent was removed under reduced pressure. The crude product was subjected to flash column chromatography on silica gel (PE/EA: 5/1) to afford 23 (44 mg, quantitative) as a colorless oil. $[\alpha]^{25} \mathrm{D}+70.8\left(c 1.10, \mathrm{CHCl}_{3}\right) ;{ }^{1} \mathrm{H} \mathrm{NMR}\left(400 \mathrm{MHz}, \mathrm{CDCl}_{3}\right) \delta$ 5.21-5.35 (m, 2H, C22-H, C23-H), 3.82-3.86 (m, 2H, C15-H, C16-H), 3.65-3.68 (m, 2H, C2H, C3-H), 3.41 (m, 1H), 2.98 (s, 3H), 2.90 (s, 3H), 2.49 (m, 2H), 2.19 (m, 2H), 1.92 (m, 1H), $1.82(\mathrm{~m}, 2 \mathrm{H}) ;{ }^{13} \mathrm{C} \mathrm{NMR}\left(100 \mathrm{MHz}, \mathrm{CDCl}_{3}\right) \delta 172.8,139.0,129.9,84.3,81.2,72.3,71.4$, 58.7, 58.3, 55.0, 45.5, 42.6, 40.9, 40.2, 38.5, 37.3, 36.7, 35.9, 35.4, 34.6, 32.3, 32.2, 32.1, 30.3, 29.7, 28.2, 22.0, 20.6, 18.9, 14.9, 14.3, 7.1, 6.9, 6.8, 5.1, 4.9, 4.8; LRMS-ESI $(\mathrm{m} / \mathrm{z})$ : $884.5\left([\mathrm{M}+\mathrm{Na}]^{+}\right)$; HRMS-MALDI $(\mathrm{m} / \mathrm{z}):[\mathrm{M}+\mathrm{Na}]^{+}$calcd for $\mathrm{C}_{49} \mathrm{H}_{95} \mathrm{NO}_{5} \mathrm{Si}_{3} 884.6410$, found 884.6412 .

\section{Reduction of 23 to 19}

To a solution of $23(350 \mathrm{mg}, 0.40 \mathrm{mmol})$ in anhydrous THF $(5 \mathrm{~mL})$ was slowly added $\mathrm{LiBHEt}_{3}(1.0 \mathrm{M}, 15.0 \mathrm{~mL})$ at $0{ }^{\circ} \mathrm{C}$. The reaction system was left to stir at ambient temperature for 16 hours before $\mathrm{MeOH}(1 \mathrm{~mL})$ was added to quench to reaction. The mixture was diluted with ethyl acetate, separated; the organic layer was dried over $\mathrm{Na}_{2} \mathrm{SO}_{4}$ and filtered. The solvent was removed under reduced pressure. The crude product was subjected to flash column chromatography on silica gel (PE/EA: 20/1) to afford 19 (266 mg, 80\%) as a colorless oil.

\section{Three-step procedure for preparing product 19}

To a solution of allylic alcohol $5(61 \mathrm{mg}, 0.077 \mathrm{mmol})$ in dry THF $(2 \mathrm{~mL})$ was added $\mathrm{NaH}(6$ $\mathrm{mg}, 60 \%, 0.11 \mathrm{mmol})$ and $\mathrm{KH}(1 \mathrm{mg})$ under argon atmosphere. The resulting solution was stirred at room temperature for $30 \mathrm{~min}$, added $\mathrm{PhSOCH}=\mathrm{CH}_{2}(0.04 \mathrm{~mL}, 0.23 \mathrm{mmol})$ in, and stirred for $18 \mathrm{~h}$ until being quenched with water. The mixture was extracted with ethyl acetate (20 $\left.\mathrm{mL}^{*} 3\right)$ and the combine organic layer was dried over $\mathrm{Na}_{2} \mathrm{SO}_{4}$. The crude product was purified by flash column chromatography on silica gel (PE/EA: 40/1, then 8/1) to recover starting material $(18 \mathrm{mg})$ and obtain desired product as colorless oil. The product was dissolved with xylene $(2 \mathrm{~mL})$, and to the solution was added $\mathrm{NaHCO}_{3}$. The resulting suspension was heated to reflux for six hours and cooled to ambient temperature. Upon being 
diluted with THF $(5 \mathrm{~mL})$ and $\mathrm{MeOH}(1 \mathrm{~mL})$, the reaction mixture was treated with $\mathrm{NaBH}_{4}$ (30 $\mathrm{mg}, 0.88 \mathrm{mmol}$ ) and stirred for $10 \mathrm{~min}$ at room temperature before being quenched with water. Diluted with EtOAc $(40 \mathrm{~mL})$, washed with brine, dried over $\mathrm{Na}_{2} \mathrm{SO}_{4}$, and filtered, the reaction solution was concentrated and subjected to flash column chromatography on silica gel (PE/EA: 20/1) to give 19 (41 mg, 65\%, 92\% based on recovering 5) as a colorless oil.

\section{Reductive removal of $\mathrm{OH}$ to get 24}

Compound 19 (500 mg, $0.61 \mathrm{mmol})$ was dissolved in dry pyridine $(2 \mathrm{~mL})$ and treated with $\mathrm{MsCl}(0.30 \mathrm{~mL}, 1.8 \mathrm{mmol})$ at ambient temperature for two hours before water was added to quench the reaction. The mixture was extracted with DCM $\left(20 \mathrm{~mL}^{*} 3\right)$; the combined organic layer was washed with brine, dried over $\mathrm{Na}_{2} \mathrm{SO}_{4}$, filtered, and concentrated under reduced pressure. The crude product was then dissolved in dry THF $\left(10 \mathrm{~mL} 0\right.$ and treated with $\mathrm{LiAlH}_{4}$ (227 mg, $6.1 \mathrm{mmol}$ ). The reaction was left to stir at $40{ }^{\circ} \mathrm{C}$ for $10 \mathrm{~h}$, quenched carefully with saturated water $(0.22 \mathrm{~mL}), 10 \% \mathrm{NaOH}(0.45 \mathrm{~mL})$, and water $(0.66 \mathrm{~mL})$. Filtration through Celite, concentration of the filtrate in vacuo, and flash column chromatography on silica gel (PE/EA: 200/1) provided the $24(380 \mathrm{mg}, 78 \%)$ as a colorless oil. $[\alpha]^{25} \mathrm{D}+7.92$ (c 2.95, $\mathrm{CHCl}_{3}$ ); IR (KBr film) 3463, 2954, 2876, 1457, 1238, 1079, 1079, 1010, 826, $745 \mathrm{~cm}^{-1} ;{ }^{1} \mathrm{H}$ NMR (400 MHz, $\left.\mathrm{CDCl}_{3}\right) \delta 5.37(\mathrm{dd}, 1 \mathrm{H}, J=8.8,15.6 \mathrm{~Hz}), 5.27(\mathrm{dd}, 1 \mathrm{H}, J=8.8,15.6 \mathrm{~Hz})$, 3.81-3.86 (m, 2H, C15-H, C16-H), 3.65-3.68 (m, 2H, C2-H, 3-H), 2.47 (m, 1H, C24-H), 2.17 $(\mathrm{d}, 1 \mathrm{H}, J=2.8 \mathrm{~Hz}) ;{ }^{13} \mathrm{C}$ NMR $\left(100 \mathrm{MHz}, \mathrm{CDCl}_{3}\right) \delta 139.1,130.8,83.1,82.1,72.3,71.4,59.4$, 58.2, 55.0, 51.2, 42.7, 40.7, 40.2, 38.5, 35.9, 35.1, 34.5, 32.3, 32.1, 31.8, 28.2, 25.2, 21.3, 20.8, 20.5, 18.8, 14.8, 14.3, 12.2, 6.9, 6.9, 6.8, 4.9, 4.8. HRMS-MALDI $(\mathrm{m} / \mathrm{z}):[\mathrm{M}+\mathrm{Na}]^{+}$calcd for $\mathrm{C}_{47} \mathrm{H}_{92} \mathrm{O}_{4} \mathrm{Si}_{3} 827.6196$, found 827.6199.

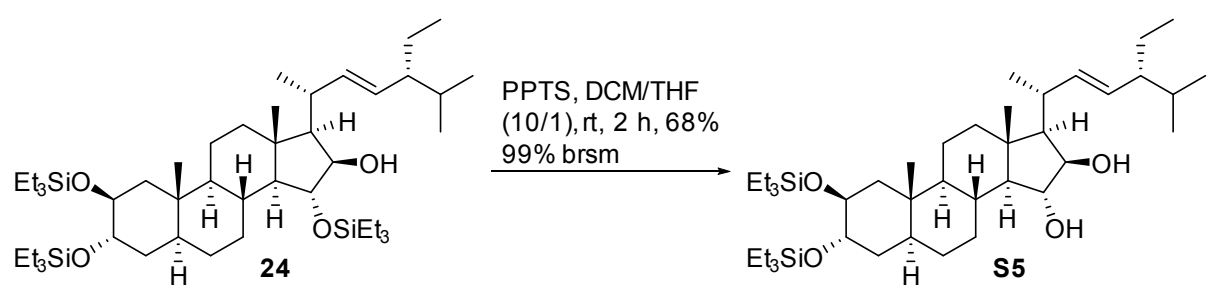

\section{Selectively deprotect C15-OSiEt 3 with PPTS}

To a solution of 24 (46 mg, $0.057 \mathrm{mmol})$ in DCM/THF $(5 \mathrm{~mL} / 0.5 \mathrm{~mL})$ was added PPTS (15 $\mathrm{mg}, 0.060 \mathrm{mmol})$. The reaction was stirred at ambient temperature for two hours and quenched with $\mathrm{Et}_{3} \mathrm{~N}(0.3 \mathrm{~mL})$. Upon removing the solvents under reduced pressure, the crude products was subjected to flash column chromatography on silica gel (PE/EA: 30/1) to recover the starting material (16 mg) and afford $\mathbf{S 5}(27 \mathrm{mg}, 68 \%, 99 \%$ based on recovering $\mathrm{SM})$ as a colorless oil. $[\alpha]^{25} \mathrm{D}+18.4\left(c 1.10, \mathrm{CHCl}_{3}\right)$; IR ( $\mathrm{KBr}$ film) 3388, 2955, 2831, 1459, 1238, 1078, 1009, $746 \mathrm{~cm}^{-1} ;{ }^{1} \mathrm{H}$ NMR $\left(300 \mathrm{MHz}, \mathrm{CDCl}_{3}\right) \delta$ 5.26-5.43 (m, 2H, C22-H, C23- 
H), 3.96 (m, 2H, C15-H, C16-H), 3.66-3.68 (m, 2H, C2-H, C3-H), 2.49 (m, 1H, C24-H), 2.33 $(\mathrm{m}, 1 \mathrm{H}, \mathrm{C} 20-\mathrm{H}) ;{ }^{13} \mathrm{C} \mathrm{NMR}\left(100 \mathrm{MHz}, \mathrm{CDCl}_{3}\right) \delta 138.6,131.4,82.6,72.2,71.4,59.7,58.4$, 55.1, 51.0, 43.7, 40.6, 40.1, 38.6, 35.9, 35.0, 34.2, 32.3, 32.0, 31.5, 30.1, 29.7, 28.1, 25.3, 21.4, 20.9, 20.5, 18.7, 14.8, 14.3, 12.3, 6.9, 6.8, 4.9, 4.8; HRMS-MALDI $(m / z):[\mathrm{M}+\mathrm{Na}]^{+}$ calcd for $\mathrm{C}_{41} \mathrm{H}_{78} \mathrm{O}_{4} \mathrm{Si}_{2} 713.5331$, found 713.5328 .

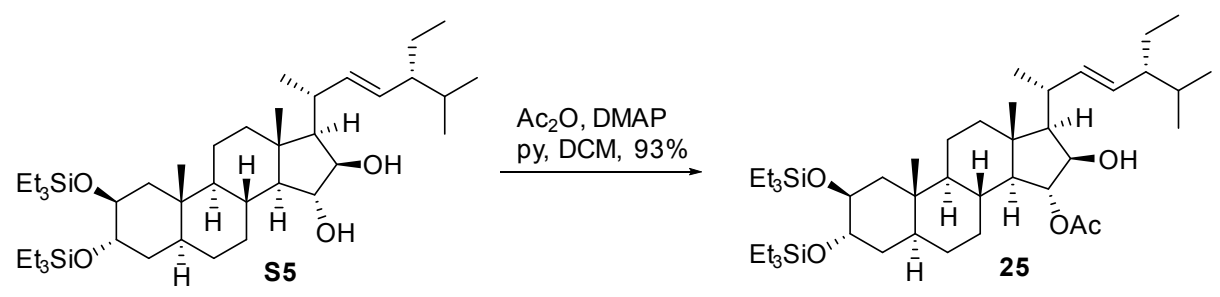

\section{Compound 25}

Diol S5 (115 mg, $0.167 \mathrm{mmol})$ was dissolved in dry DCM (4 mL), treated with pyridine (0.5 $\mathrm{mL}), \mathrm{Ac}_{2} \mathrm{O}(0.10 \mathrm{~mL})$, and DMAP $(1 \mathrm{mg})$. The solution was left to stir at ambient temperature for two hours, quenched with water, and extracted with DCM (10 mL*2). The combined organic layer was dried over $\mathrm{Na}_{2} \mathrm{SO}_{4}$, filtered, and concentrated in vacuo. Flash column chromatography on silica gel (PE/EA: 80/1) afforded 25 (120 $\mathrm{mg}, 95 \%)$ as a colorless oil. $[\alpha]^{25} \mathrm{D}+29.8$ (c 1.00, $\left.\mathrm{CHCl}_{3}\right)$; IR (KBr film) 3555, 2957, 2875, 1724, 1459, 1258, 1080, 1017, 801, $745 \mathrm{~cm}^{-1} ;{ }^{1} \mathrm{H}$ NMR $\left(300 \mathrm{MHz}, \mathrm{CDCl}_{3}\right) \delta 5.40(\mathrm{dd}, 1 \mathrm{H}, J=8.1,15.6$ $\mathrm{Hz}), 5.20(\mathrm{dd}, 1 \mathrm{H}, J=8.1,15.6 \mathrm{~Hz}), 4.62-4.69(\mathrm{~m}, 1 \mathrm{H}, \mathrm{C} 15-\mathrm{H}), 3.87(\mathrm{~m}, 1 \mathrm{H}, \mathrm{C} 16-\mathrm{H})$, 3.63-3.72 (m, 2H, C2-H, C3-H), 2.70 (d, $J=2.0 \mathrm{~Hz}, 1 \mathrm{H}), 2.51-2.65(\mathrm{~m}, 1 \mathrm{H}, \mathrm{C} 24-\mathrm{H}, \mathrm{C} 20-\mathrm{H})$, 2.04 (s, 3H); ${ }^{13} \mathrm{C}$ NMR $\left(100 \mathrm{MHz}, \mathrm{CDCl}_{3}\right) \delta 172.3,137.5,130.5,86.8,79.9,72.2,71.4,59.2$, 56.8, 54.9, 51.2, 43.3, 40.4, 40.1, 38.6, 35.9, 34.0, 33.5, 32.0, 31.6, 31.5, 31.4, 30.2, 28.1, 25.3, 21.3, 20.9, 20.5, 20.3, 18.8, 14.5, 14.3, 12.2, 6.9, 4.9, 4.8; LRMS-ESI $(m / z): 755.8$ ([M $\left.+\mathrm{Na}]^{+}\right)$; HRMS-MALDI $(\mathrm{m} / \mathrm{z}):[\mathrm{M}+\mathrm{Na}]^{+}$calcd for $\mathrm{C}_{43} \mathrm{H}_{80} \mathrm{O}_{5} \mathrm{Si}_{2}$ 755.5436, found 755.5427.
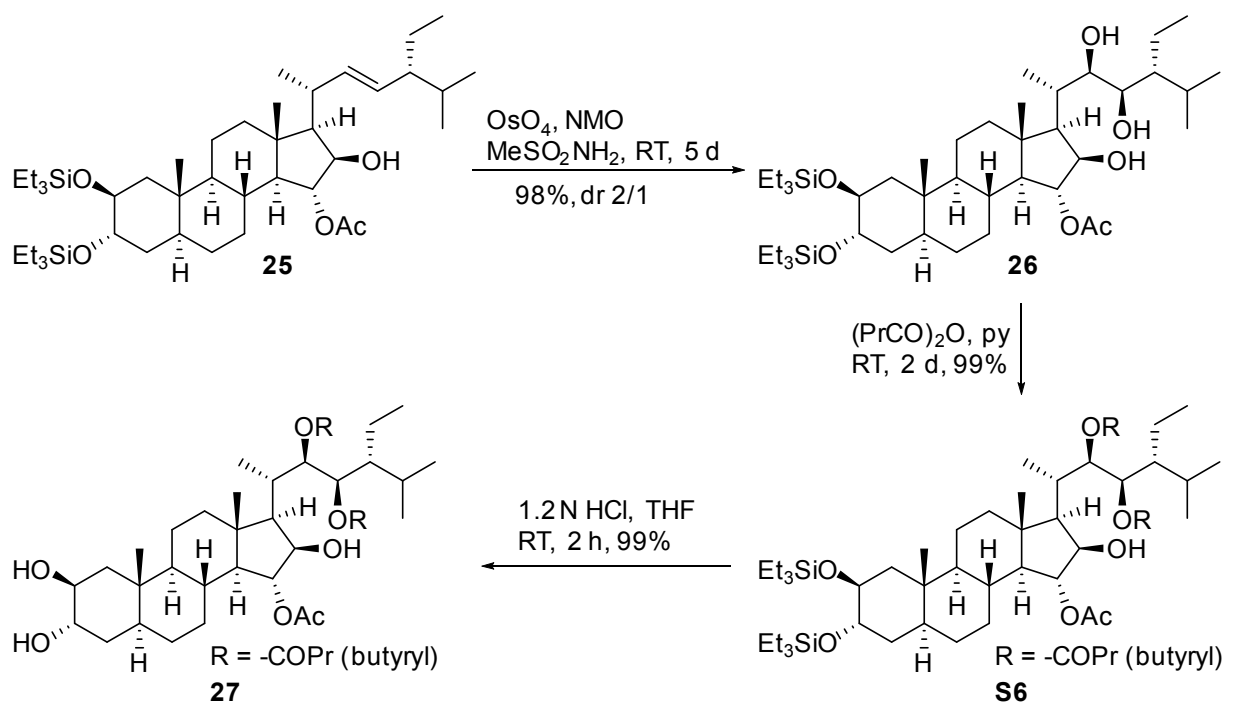


\section{Dihydroxylation to give 26}

To a solution of alkene $25(118 \mathrm{mg}, 0.161 \mathrm{mmol})$ in THF/tBuOH $(10 \mathrm{~mL} / 10 \mathrm{~mL})$ were added NMO (50\% wt/V, $0.20 \mathrm{~mL}, 0.805 \mathrm{mmol}), \mathrm{OsO}_{4}(1.0 \mathrm{~g} / \mathrm{L}, 3 \mathrm{~mL}, 3 \mathrm{mg}, 0.012 \mathrm{mmol})$, and $\mathrm{MeSO}_{2} \mathrm{NH}_{2}(30 \mathrm{mg}, 0.322 \mathrm{mmol}$ ) at ambient temperature. The solution was left to stir for five days until saturated $\mathrm{Na}_{2} \mathrm{SO}_{3}$ aqueous solution was added to quench the reaction. The organic solvents were removed under reduced pressure, and the residue was dissolved with ethyl acetate $(50 \mathrm{~mL})$. After separation, the organic layer was dried over $\mathrm{Na}_{2} \mathrm{SO}_{4}$, filtered, and concentrated in vacuo. Flash column chromatography on silica gel (PE/EA: 80/1, then 4/1) recovered starting material $25(58 \mathrm{mg})$ and afforded an inseparable mixture of $\mathbf{2 6}$ and 22,23diepi-26 (26/isomer: 2/1 by NMR, $58 \mathrm{mg}, 50 \%, 98 \%$ based on recovering SM) as colorless oil.

\section{Compound S6}

Added butyric anhydride (1 mL), a solution of 26/22,23-diepi-26 (2/1, $43 \mathrm{mg}, 0.024 \mathrm{mmol})$ and DMAP $(1 \mathrm{mg})$ in dry pyridine/DCM $(1 \mathrm{~mL} / 1 \mathrm{~mL})$ was stirred at ambient temperature for $48 \mathrm{~h}$ before the solvent was removed in vacuo. The residue was purified with flash column chromatography on silica gel (PE/EA: 60/1) to give S6 (34 mg, 67\%, 99\% according to the amount of 26) as a colorless oil. $[\alpha]^{25} \mathrm{D}+42.1$ (c 1.75, $\left.\mathrm{CHCl}_{3}\right)$; IR ( $\mathrm{KBr}$ film) 3538, 2957, 2876, 1739, 1459, 1370, 1254, 1077, 1045, 947, $746 \mathrm{~cm}^{-1} ;{ }^{1} \mathrm{H}$ NMR (400 MHz, $\left.\mathrm{CDCl}_{3}\right) \delta$ $5.46(\mathrm{~d}, 1 \mathrm{H}, J=9.2 \mathrm{~Hz}), 5.35(\mathrm{~d}, 1 \mathrm{H}, J=9.2 \mathrm{~Hz}), 4.45(\mathrm{dd}, 1 \mathrm{H}, J=11.2,2.0 \mathrm{~Hz}, \mathrm{C} 15-\mathrm{H})$, 4.02-4.09 (m, 1H, C16-H), 3.65-3.68 (m, 2H, C2-H, C3-H), 2.12-2.32 (m, 6H), $2.06(\mathrm{~s}, 3 \mathrm{H})$, $0.83(\mathrm{~d}, 3 \mathrm{H}, J=6.8 \mathrm{~Hz}) .{ }^{13} \mathrm{C} \mathrm{NMR}\left(100 \mathrm{MHz}, \mathrm{CDCl}_{3}\right) \delta 173.2,88.2,78.9,75.3,74.5,72.2$, $71.3,56.2$, 54.7, 54.5, 45.6, 43.0, 40.1, 38.5, 36.5, 36.3, 35.8, 34.2, 31.7, 30.7, 28.0, 26.1, 23.4, 21.2, 20.5, 18.6, 18.3, 18.2, 17.9, 14.3, 14.2, 13.8, 13.7, 12.6, 12.1, 6.9, 4.9, 4.8; LRMS-ESI $(m / z): 929.5\left([\mathrm{M}+\mathrm{Na}]^{+}\right)$; HRMS-MALDI $(m / z):[\mathrm{M}+\mathrm{Na}]^{+}$calcd for $\mathrm{C}_{51} \mathrm{H}_{94} \mathrm{O}_{9} \mathrm{Si}_{2}$ 929.6328, found 929.6301 .

\section{Compound 27}

Compound S6 (23 mg, $0.025 \mathrm{mmol})$ was dissolved in THF $(2.0 \mathrm{~mL})$ and to the solution was added concentrated $\mathrm{HCl}(1 \mathrm{~mL})$. The reaction mixture was stirred at ambient temperature for two hours, diluted with ethyl acetate $(30 \mathrm{~mL})$, washed with brine, dried over $\mathrm{Na}_{2} \mathrm{SO}_{4}$, filtered, and concentrated in vacuo. The crude product was purified with flash column chromatography on silica gel (PE/EA: $2 / 1)$ to give $27(18 \mathrm{mg}, 99 \%)$ as a colorless oil. $[\alpha]^{25} \mathrm{D}$ + 59.9 (c 0.25, $\mathrm{CHCl}_{3}$ ); IR (KBr film) 3527, 2961, 2874, 1732, 1462, 1369, 12594, 1041, 800, $757 \mathrm{~cm}^{-1} ;{ }^{1} \mathrm{H}$ NMR (400 MHz, $\left.\mathrm{CDCl}_{3}\right) \delta 5.46(\mathrm{~d}, 1 \mathrm{H}, J=9.2 \mathrm{~Hz}), 5.35$ (d, $1 \mathrm{H}, J=9.2$ $\mathrm{Hz}$ ), 4.45 (dd, 1H, $J=11.2,2.0 \mathrm{~Hz}, \mathrm{C} 15-\mathrm{H}), 4.02-4.09$ (m, 1H, C16-H), 3.83-3.90 (m, 2H, C2-H, C3-H), 3.11 (s, 1H), 2.12-2.32 (m, 6H), 2.05 (s, 3H), 0.83 (d, 3H, $J=6.8 \mathrm{~Hz}) ;{ }^{13} \mathrm{C}$ NMR (100 MHz, $\left.\mathrm{CDCl}_{3}\right) \delta 173.2,88.0,78.8,75.3,74.5,71.6,70.5,56.2,54.6,54.5,45.6$, 43.0, 40.3, 38.6, 36.5, 36.3, 35.7, 34.2, 31.6, 31.4, 30.7, 29.6, 27.9, 26.1, 23.4, 21.2, 20.5, 
18.6, 18.3, 18.2, 17.9, 14.5, 14.2, 13.8, 13.7, 12.6, 12.1; LRMS-ESI $(m / z): 701.2\left([\mathrm{M}+\mathrm{Na}]^{+}\right)$; HRMS-MALDI $(\mathrm{m} / \mathrm{z})$ : $[\mathrm{M}+\mathrm{Na}]^{+}$calcd for $\mathrm{C}_{39} \mathrm{H}_{66} \mathrm{O}_{9} 701.4601$, found 701.4599.

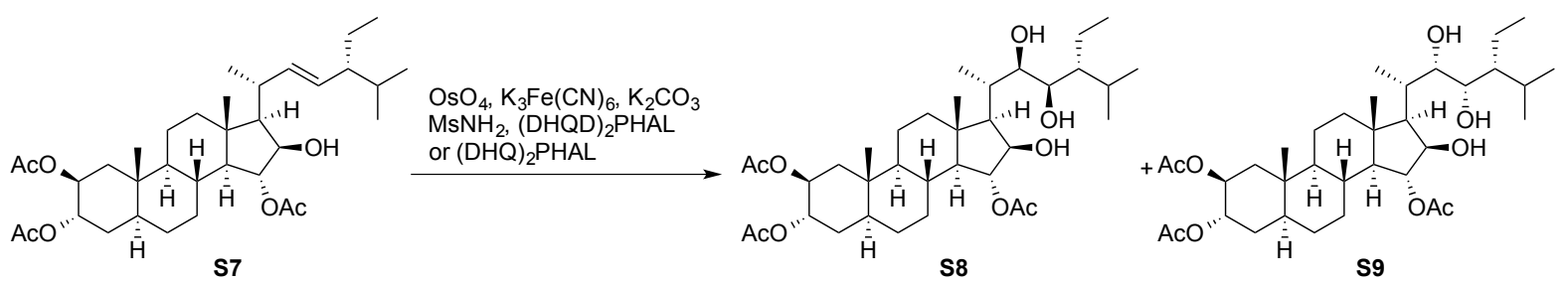

\section{Dihydroxylation of C22-C23 double bond}

To a solution of $\mathbf{S 7}$ (90 $\mathrm{mg}, 0.15 \mathrm{mmol}$; prepared from 24 in 2 steps and 94\% yield: TBAF, THF, reflux, $2 \mathrm{~h}$; then $\mathrm{Ac}_{2} \mathrm{O}$, pyridine, $\left.3 \mathrm{~h}\right)$ in THF/t-BuOH $(10 \mathrm{~mL} / 10 \mathrm{~mL})$ were added NMO (300 mg, $2.54 \mathrm{mmol}), \mathrm{OsO}_{4}(1.0 \mathrm{~g} / \mathrm{L}$ in water, $18 \mathrm{~mL}, 18 \mathrm{mg}, 0.07 \mathrm{mmol})$, and $\mathrm{MeSO}_{2} \mathrm{NH}_{2}$ (200 mg, $2.1 \mathrm{mmol}$ ) at ambient temperature. The solution was stired for five days and quenched with saturated aqueous $\mathrm{Na}_{2} \mathrm{SO}_{3}$ solution. The organic solvents were removed under reduced pressure, and the residue was extracted with ethyl acetate $(30 \mathrm{~mL})$ for three times. The combined organic layers were washed with water and brine, dried over $\mathrm{Na}_{2} \mathrm{SO}_{4}$, filtered, concentrated under reduced pressure, and purified with flash column chromatography on silica gel (PE/EA: 4/1 to 3/1) to give $\mathbf{S 8}(60 \mathrm{mg}, 64 \%)$ as colorless oil and S9 (less polar, 29 $\mathrm{mg}, 31 \%$ ) as colorless oil.

Under standard Sharpless asymmetric dihydroxylation conditions, the dihydroxylation of $\mathbf{S 7}$ still needed five days to completion and gave lower overall yields (58-70\%). Using chiral ligand (DHQD) 2 PHAL (1.0 equiv), $\mathrm{OsO}_{4}$ ( 0.5 equiv), $\mathrm{K}_{3} \mathrm{Fe}(\mathrm{CN})_{6}$ (6 equiv), $\mathrm{K}_{2} \mathrm{CO}_{3}$ (6 equiv), $\mathrm{MsNH}_{2}$ (2 equiv), the ratio of $\mathbf{S 8} / \mathbf{S 9}$ improved to 2.6-2.9/1; while changing the chiral ligand to (DHQ) ${ }_{2} \mathrm{PHAL}$, the ratio of $\mathbf{S 8} / \mathbf{S 9}$ was reversed to $1 / 2-3$.

Compound S8: $[\alpha]^{25} \mathrm{D}+54.2\left(c\right.$ 1.0, $\left.\mathrm{CHCl}_{3}\right) ;{ }^{1} \mathrm{H}$ NMR $\left(400 \mathrm{MHz}, \mathrm{CDCl}_{3}\right) \delta$ 4.85-4.91 (m, 2H, C2-H, C3-H), 4.44 (dd, $J=10.8,1.6 \mathrm{~Hz}, 1 \mathrm{H}, \mathrm{C} 15-\mathrm{H}), 4.06$ (dd, $J=7.6,2.0 \mathrm{~Hz}, 1 \mathrm{H}, \mathrm{C} 16-$ H), 3.74-3.78 (m, 1H, C22-H), 3.54-3.58 (m, 1H, C23-H), 3.52 (br s, 1H), 3.09 (s, 1H), 2.21-2.33 (m, 2H), 2.06 (s, 3H), $2.04(\mathrm{~s}, 3 \mathrm{H}), 2.03(\mathrm{~s}, 3 \mathrm{H}) ;{ }^{13} \mathrm{C} \mathrm{NMR}\left(100 \mathrm{MHz}, \mathrm{CDCl}_{3}\right) \delta$ 173.5, 169.9, 169.7, 88.5, 81.2, 79.8, 79.1, 70.3, 69.9, 56.5, 55.6, 54.6, 48.5, 43.3, 40.2, 39.4, $37.8,35.5,34.4,31.5,29.3,27.9,27.8,27.6,27.4,21.5,21.4,21.4,20.7,19.5,19.3,14.5$, 13.9, 13.8, 12.3; HRMS-ESI $(m / z)$ : $[\mathrm{M}+\mathrm{Na}]^{+}$calcd for $\mathrm{C}_{35} \mathrm{H}_{58} \mathrm{O}_{9} 645.3973$, found 645.3978.

Compound S9: $[\alpha]^{25} \mathrm{D}+41.1$ (c 1.05, $\left.\mathrm{CHCl}_{3}\right)$; IR (KBr film) 3482, 2925, 2852, 1740, 1369 , 1254, 1033, $757 \mathrm{~cm}^{-1} ;{ }^{1} \mathrm{H}$ NMR (400 MHz, CDCl 3 ) $\delta$ 4.85-4.91 (m, 2H, C2-H, C3-H), 4.49 $(\mathrm{dd}, J=6.8,2.4 \mathrm{~Hz}, 1 \mathrm{H}, \mathrm{C} 15-\mathrm{H}), 4.13(\mathrm{dd}, J=8.0,2.4 \mathrm{~Hz}, 1 \mathrm{H}, \mathrm{C} 16-\mathrm{H}), 4.03$ (br s, $1 \mathrm{H}, \mathrm{OH})$,

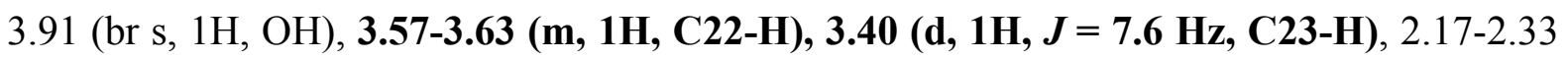
$(\mathrm{m}, 2 \mathrm{H}), 2.06(\mathrm{~s}, 3 \mathrm{H}), 2.05(\mathrm{~s}, 3 \mathrm{H}), 2.04(\mathrm{~s}, 3 \mathrm{H}), 0.83(\mathrm{~d}, 3 \mathrm{H}, J=6.8 \mathrm{~Hz}) ;{ }^{13} \mathrm{C} \mathrm{NMR}(100$ $\left.\mathrm{MHz}, \mathrm{CDCl}_{3}\right) \delta 173.8,169.9,169.8,87.6,80.8,72.9,70.3,69.9,57.2,56.2,54.5,49.2,44.4$, $40.5,39.4,37.8,35.5,34.4,34.2,31.5,29.8,29.3,28.3,27.8,21.4,21.4,21.2,20.9,20.7$, 
19.4, 18.4, 16.2, 15.1, 14.5, 13.9; HRMS-ESI $(m / z):[\mathrm{M}+\mathrm{Na}]^{+}$calcd for $\mathrm{C}_{35} \mathrm{H}_{58} \mathrm{O}_{9} 645.3973$, found 645.3969 .
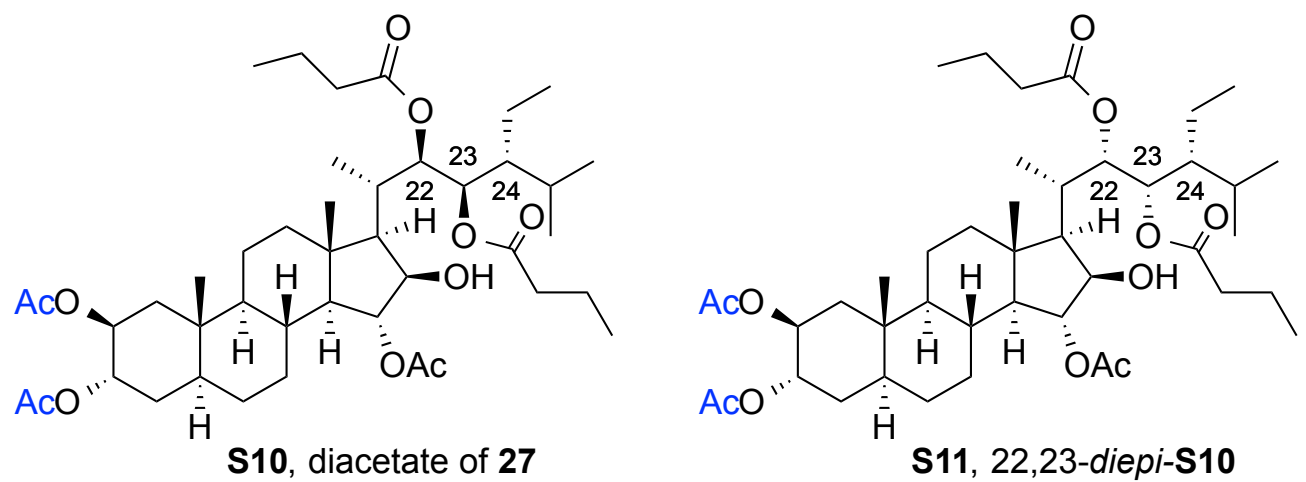

\section{S10 and S11}

To a solution of $\mathbf{S 8}(20 \mathrm{mg}, 0.032 \mathrm{mmol})$ in DCM $(1 \mathrm{~mL})$ were added pyridine $(1 \mathrm{~mL})$, butyric anhydride $(1 \mathrm{~mL})$, and DMAP $(1 \mathrm{mg})$. The mixture was stirred at ambient temperature for $24 \mathrm{~h}$ and quenched with water; the solvent was removed in vacuo. The residue was purified with flash column chromatography on silica gel (PE/EA: 7/1) to give $\mathbf{S 1 0}$ (also diacetate of 27, $24 \mathrm{mg}, 99 \%$ ) as a colorless oil. S11, which is the 22,23-diepi-S10, was prepared from $\mathbf{S 9}$ in $84 \%$ yield as a colorless oil.

S10: $[\alpha]^{25} \mathrm{D}+59.3\left(c\right.$ 0.20, $\left.\mathrm{CHCl}_{3}\right)$; IR (KBr film) 3555, 2957, 2875, 1724, 1459, 1258, 1080, 1017, 801, $745 \mathrm{~cm}^{-1}$; ${ }^{1} \mathrm{H}$ NMR (400 MHz, $\left.\mathrm{CDCl}_{3}\right) \delta 5.46(\mathrm{~d}, 1 \mathrm{H}, J=8.8 \mathrm{~Hz}, \mathrm{C} 22-\mathrm{H}), 5.35$ (d, $1 \mathrm{H}, J=8.8 \mathrm{~Hz}, \mathrm{C} 23-\mathrm{H}), 4.86-4.89$ (m, 2H, C2-H, C3-H), 4.457 (dd, 1H, J = 10.7, $2.0 \mathrm{~Hz}$, C15-H), 4.07 (dd, 1H, $J=8.0,2.0 \mathrm{~Hz}, \mathrm{C} 16-\mathrm{H}), 3.08(\mathrm{~s}, 1 \mathrm{H}, \mathrm{C} 16-\mathrm{OH}), 2.21-2.28(\mathrm{~m}, 6 \mathrm{H})$, 2.05 (s, 3H), 2.05 (s, 3H), 2.04 (s, 3H), 0.84 (s, 3H, C18-H); ${ }^{1} \mathrm{H}$ NMR (400 MHz, DMSO) $\delta$ 5.36 (d, 1H, $J=9.2 \mathrm{~Hz}, \mathrm{C} 22-\mathrm{H}), 5.25$ (d, 1H, $J=9.2 \mathrm{~Hz}, \mathrm{C} 23-\mathrm{H}), 5.00$ (d, 1H, C16-OH), 4.82 (dd, 1H, $J=2.4,10.8 \mathrm{~Hz}, \mathrm{C} 15-\mathrm{H}), 4.71-4.77$ (m, 2H, C2-H, C3-H), 3.94 (ddd, 1H, $J=$ 10.8, 5.2, 2.4 Hz, C16-H), 2.12-2.32 (m, 6H), 2.03 (s, 3H), 1.99 (s, 3H), 1.96 (s, 3H), 0.85 (s, $3 \mathrm{H}, \mathrm{C} 18-\mathrm{H}), 0.79$ (d, $J=6.8 \mathrm{~Hz}, \mathrm{C} 3-\mathrm{H}) ;{ }^{13} \mathrm{C} \mathrm{NMR}\left(100 \mathrm{MHz}, \mathrm{CDCl}_{3}\right) \delta 173.2,173.1,172.2$, $169.7,169.6,86.7,79.2,74.7,72.3,70.1,69.8,56.6,54.4,54.3,47.3,43.8,40.0,39.3,37.6$, $36.7,36.4,35.3,34.1,32.6,31.1,29.6,29.1,28.3,27.6,21.2,21.2,20.4,20.2,19.0,18.4$, 18.3, 18.1, 14.3, 13.7, 13.7; HRMS-MALDI $(\mathrm{m} / \mathrm{z})$ : $[\mathrm{M}+\mathrm{Na}]^{+}$calcd for $\mathrm{C}_{43} \mathrm{H}_{70} \mathrm{O}_{11} 785.4810$, found 785.4816 .

S11: $[\alpha]^{25} \mathrm{D}+34.1\left(c\right.$ 0.70, $\left.\mathrm{CHCl}_{3}\right)$; IR (KBr film) 3534, 2960, 2933, 1736, 1436, 1369, 1249, 1034, $756 \mathrm{~cm}^{-1}$; ${ }^{1} \mathrm{H}$ NMR (400 MHz, $\left.\mathrm{CDCl}_{3}\right) \delta$ 5.33-5.35 (m, 1H, C22-H), 5.26-5.28 (m, 1H, C23-H), 4.86-4.92 (m, 2H, C2-H, C3-H), 4.64 (d, 1H, J=10.0 Hz, C15-H), 3.93 (d, 1H, J= 7.6 Hz, C16-H), 3.30 (s, 1H, C16-OH), 2.27-2.34 (m, 6H), 2.07 (s, 3H), $2.04(2 \mathrm{~s}, 6 \mathrm{H}) ;{ }^{13} \mathrm{C}$ NMR $\left(100 \mathrm{MHz}, \mathrm{CDCl}_{3}\right) \delta 173.2,173.1,172.2,169.7,169.6,86.7,79.2,74.7,72.3,70.1$, 69.8, 56.6, 54.4, 54.3, 47.3, 43.8, 40.0, 39.3, 37.6, 36.7, 36.4, 35.3, 34.1, 32.6, 31.1, 29.6, 29.1, 28.3, 27.6, 21.2, 21.2, 20.4, 20.2, 19.0, 18.4, 18.3, 18.1, 14.3, 13.7, 13.7; HRMSMALDI $(\mathrm{m} / \mathrm{z})$ : $[\mathrm{M}+\mathrm{Na}]^{+}$calcd for $\mathrm{C}_{43} \mathrm{H}_{70} \mathrm{O}_{11} 785.4810$, found 785.4816 . 

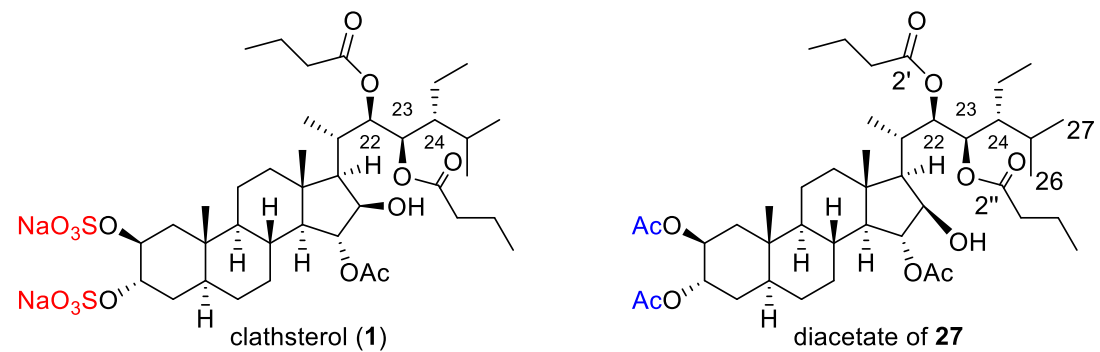

Table 3. Comparing 1H NMR Data of clathesterol and 27/S10

\begin{tabular}{|c|c|c|c|c|}
\hline & $\begin{array}{c}\text { Clathsterol 1 (DMSO, 500 } \\
\text { MHz) }\end{array}$ & $\begin{array}{c}\mathbf{2 7} \text { diacetate/S10 (DMSO, } \\
400 \mathrm{MHz})\end{array}$ & $\begin{array}{c}\mathbf{2 7} \text { diacetate/S10 } \\
\left(\mathrm{CDCl}_{3}, 400 \mathrm{MHz}\right)\end{array}$ & $\mathbf{2 7}$ \\
\hline 15 & $4.80 \mathrm{dd}(10.6,2.3)$ & $4.82 \mathrm{dd}(10.8,2.4)$ & $4.45 \mathrm{dd}(10.7,2.0)$ & $4.45 \mathrm{dd},(10.8,2.0)$ \\
\hline 16 & $3.94 \mathrm{ddd}(10.6,4.8,2.3)$ & $3.94 \mathrm{ddd}(10.8,5.2,2.4)$ & $4.07 \mathrm{dd}(8.0,2.0)$ & $4.05 \mathrm{dd}(7.8,2.0)$ \\
\hline 18 & $0.85 \mathrm{~s}$ & $0.85 \mathrm{~s}$ & $0.84 \mathrm{~s}$ & $0.84 \mathrm{~s}$ \\
\hline 22 & $5.38 \mathrm{~d}(9.1)$ & $5.36 \mathrm{~d}(9.2)$ & $5.46 \mathrm{~d}(8.8)$ & $5.46 \mathrm{~d}(9.2)$ \\
\hline 23 & $5.25 \mathrm{~d}(9.1,1.4)$ & $5.25 \mathrm{dd}(9.2,0.8)$ & $5.35 \mathrm{~d}(8.8)$ & $5.35 \mathrm{~d}(9.2)$ \\
\hline 26 & $0.86 \mathrm{~d}(6.5, \mathrm{Me})$ & $0.85 \mathrm{~d}(6.8)$ & 0.85 & 0.85 \\
\hline 27 & $0.79 \mathrm{~d}(6.5, \mathrm{Me})$ & $0.79 \mathrm{~d}(6.8)$ & $0.84 \mathrm{~d}(6.8)$ & $0.83 \mathrm{~d}(6.8)$ \\
\hline 2 & $2.12,2.14$ & $2.12,2.18$ & $2.17-2.31$ & $2.12-2.32$ \\
\hline $2 ”$ & $2.25,2.18$ & $2.32,2.22$ & $2.17-2.31$ & $2.12-2.32$ \\
\hline $15-O A c$ & $1.92 \mathrm{~s}$ & $1.94 \mathrm{~s}$ & $2.04 \mathrm{~s}$ & $2.06 \mathrm{~s}$ \\
\hline
\end{tabular}




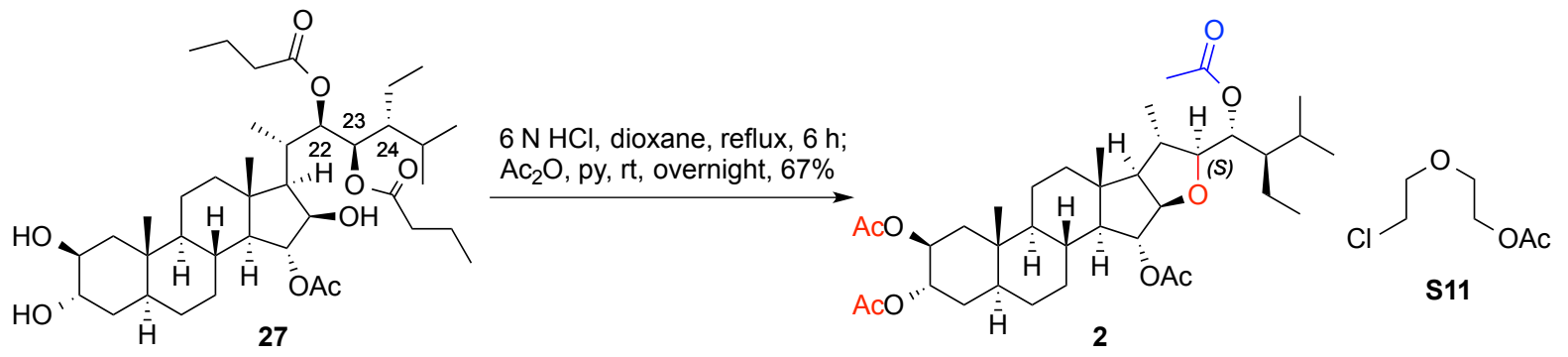

Acid hydrolysis of 27

To a flask with compound 27 (36 mg, $0.053 \mathrm{mmol}$ ) was added $6 \mathrm{~N}$ aqueous $\mathrm{HCl}$ solution (6 $\mathrm{mL}$ ) and the mixture was heated to reflux. Because 27 was not soluble in water, dioxane (6 $\mathrm{mL}$, bp $110{ }^{\circ} \mathrm{C}$ ) was then added. The solution was refluxing for $6 \mathrm{~h}$ and concentrated at reduced pressure to remove the solvents. The crude was co-evaporated with toluene and dissolved in dry pyridine $(2 \mathrm{~mL}) . \mathrm{Ac}_{2} \mathrm{O}(1 \mathrm{~mL})$ was added and the mixture was stirred at ambient temperature overnight. The solvent was removed under reduced pressure. Purification by flash column chromatography twice (PE/EA: 4/1) on silica gel gave 28 (first column chromatography gave $34 \mathrm{mg}$, second chromatography gave $23 \mathrm{mg}, 67 \%$ ) as a white foam. (Using dioxane in acid hydrolysis step caused the formation of an inseparable side product, 2-(2-chloroethoxy)ethyl acetate S11 ${ }^{\circ}$. NOESY crosspeaks of C22-H and C16-H/ C21-Me proved C22 is $\boldsymbol{S}$-configured. $\mathrm{R}_{\mathrm{f}}=0.5$ (silica gel, EtOAc/hexane: 1/4); IR (film): 1961, 1740, 1366, 1033, $970 \mathrm{~cm}^{-1}$; ${ }^{1} \mathrm{H}$ NMR (400 MHz, $\left.\mathrm{CDCl}_{3}\right) \delta 5.24$ (dd, $J=8.6,2.6 \mathrm{~Hz}$, 1H, C23-H), 5.11 (d, $J=10.6,2.2 \mathrm{~Hz}, 1 \mathrm{H}, \mathrm{C} 15-\mathrm{H}$ ), 4.89 (br s, 1H, C2-H), 4.87 (br s, 1H, C3H), 4.14 (dd, $J=7.6,2.2 \mathrm{~Hz}, 1 \mathrm{H}, \mathrm{C} 16-\mathrm{H}), 3.52$ (dd, $J=8.6,6.9 \mathrm{~Hz}, 1 \mathrm{H}, \mathrm{C} 22-\mathrm{H}), 2.06$ (s, $3 \mathrm{H}), 2.05$ (s, 3H), $2.05(\mathrm{~s}, 3 \mathrm{H}), 2.02(\mathrm{~s}, 3 \mathrm{H}), 1.01$ (d, $J=7.0 \mathrm{~Hz}, 3 \mathrm{H}), 0.90-0.95(9 \mathrm{H}, 3 \mathrm{Me})$, 0.88 (s, 3H), 0.85 (d, $J=7.0 \mathrm{~Hz}, 3 \mathrm{H}) ;{ }^{13} \mathrm{C} \mathrm{NMR}\left(100 \mathrm{MHz}, \mathrm{CDCl}_{3}\right) \delta 170.6,170.4,169.9$, $169.8,90.3,87.3,80.2,75.8,70.2,69.9,63.7,59.7,54.7,46.3,42.3,39.4,39.4,37.9,36.0$, 35.6, 34.6, 31.0, 29.3, 27.8, 26.6, 23.0, 21.7, 21.5, 21.3, 21.2, 20.4, 18.7, 18.1, 17.1, 13.9, 12.9; MS-ESI $(\mathrm{m} / \mathrm{z}): 669.3\left([\mathrm{M}+\mathrm{Na}]^{+}\right)$; HRMS-ESI $(\mathrm{m} / \mathrm{z}):[\mathrm{M}+\mathrm{Na}]^{+}$calcd for $\mathrm{C}_{37} \mathrm{H}_{58} \mathrm{O}_{9}$ : 669.8512, found: 669.8528 .

\footnotetext{
${ }^{6}$ The data of $\mathbf{S 1 1}$ we collected from the NMR of $\mathbf{2 8}:{ }^{1} \mathrm{H}$ NMR (400 MHz, $\left.\mathrm{CDCl}_{3}\right) \delta 4.25$ (t, $2 \mathrm{H}, J=4.5 \mathrm{~Hz}$ ), $3.77(\mathrm{t}, 2 \mathrm{H}, J=5.7 \mathrm{~Hz}), 3.74(\mathrm{t}, 2 \mathrm{H}, J=4.5 \mathrm{~Hz}), 3.65(\mathrm{t}, 2 \mathrm{H}, J=6.0 \mathrm{~Hz}), 2.11(\mathrm{~s}, 3 \mathrm{H}) ;{ }^{13} \mathrm{C}$ NMR $\left(\mathrm{CDCl}_{3}\right) \delta$ 71.6, 69.5, 63.7, 42.9, 21.4 (the CO peak was not showed). The reported data for S11: ${ }^{1} \mathrm{H}$ NMR $\left(\mathrm{CDCl}_{3}\right) \delta 4.26$ $(\mathrm{t}, 2 \mathrm{H}, J=4.7 \mathrm{~Hz}), 3.79(\mathrm{t}, 2 \mathrm{H}, J=6.3 \mathrm{~Hz}), 3.74(\mathrm{t}, 2 \mathrm{H}, J=4.7 \mathrm{~Hz}), 3.65(\mathrm{t}, 2 \mathrm{H}, J=6.3 \mathrm{~Hz}), 2.11(\mathrm{~s}, 3 \mathrm{H}) ;{ }^{13} \mathrm{C}$ NMR $\left(\mathrm{CDCl}_{3}\right) \delta 171.3,71.6,69.5,63.7,42.9,21.4$. Ballut, S.; Naud-Martin, D.; Loock, B.; Maillard, P. J. Org. Chem. 2011, 76, 2010-2028.
} 
Table 4. Comparing 1H NMR Data of 28 with 2

\begin{tabular}{ccccccc} 
No. & Reported 2 & Synthetic 28 & & No. & Reported 2 & Synthetic 28 \\
\cline { 1 - 3 } 2 & $4.90 \mathrm{bs}$ & $4.89 \mathrm{bs}$ & & 19 & $0.89 \mathrm{~s}$ & $0.88 \mathrm{~s}$ \\
3 & $4.87 \mathrm{bs}$ & $4.87 \mathrm{bs}$ & 22 & $3.52 \mathrm{dd}(8.7,6.9)$ & $3.54 \mathrm{dd}(8.6,6.9)$ \\
15 & $5.11 \mathrm{dd}(10.6,2.1)$ & $5.11 \mathrm{dd}(10.6,2.2)$ & 23 & $5.27 \mathrm{dd}(8.7,2.7)$ & $5.24 \mathrm{dd}(8.6,2.6)$ \\
16 & $4.14 \mathrm{dd}(7.7,2.1)$ & $4.14 \mathrm{dd}(7.6,2.2)$ & & &
\end{tabular}

Table 5. Comparing 13C NMR Data of 28 with 2

\begin{tabular}{|c|c|c|c|c|c|}
\hline No. & Reported 2 & Synthetic 28 & No. & Reported 2 & Synthetic 28 \\
\hline 1 & 37.7 & 37.9 & 19 & 12.8 & 12.9 \\
\hline 2 & 70.1 & 70.2 & 20 & 35.8 & 36.0 \\
\hline 3 & 69.8 & 69.9 & 21 & 18.6 & 18.7 \\
\hline 4 & 29.7 & 29.3 & 22 & 87.2 & 87.3 \\
\hline 5 & 39.2 & 39.4 & 23 & 75.4 & 75.8 \\
\hline 6 & 30.9 & 31.0 & 24 & 46.3 & 46.3 \\
\hline 7 & 27.6 & 27.8 & 25 & 26.5 & 26.6 \\
\hline 8 & 34.5 & 34.6 & 26 & 22.9 & 23.0 \\
\hline 9 & 54.6 & 54.7 & 27 & 18.0 & 18.1 \\
\hline 10 & 35.4 & 35.6 & 28 & 18.6 & 18.7 \\
\hline 11 & 20.2 & 20.4 & 29 & 13.8 & 13.9 \\
\hline 12 & 39.3 & 39.4 & $1^{\prime}$ & 170.1 & 170.6 \\
\hline 13 & 42.1 & 42.3 & $2^{\prime}$ & 36.4 & 21.7 \\
\hline 14 & 59.7 & 59.7 & $3^{\prime}$ & 18.3 & not available \\
\hline 15 & 80.1 & 80.2 & $4^{\prime}$ & 13.9 & not available \\
\hline 16 & 90.2 & 90.3 & $2 \mathrm{Ac}$ & $168.4,21.5$ & $169.9,21.5$ \\
\hline 17 & 63.7 & 63.7 & $3 \mathrm{Ac}$ & $168.3,21.3$ & $169.8,21.3$ \\
\hline 18 & 17.0 & 17.1 & $15 \mathrm{Ac}$ & $168.3,21.3$ & $170.4,21.2$ \\
\hline
\end{tabular}



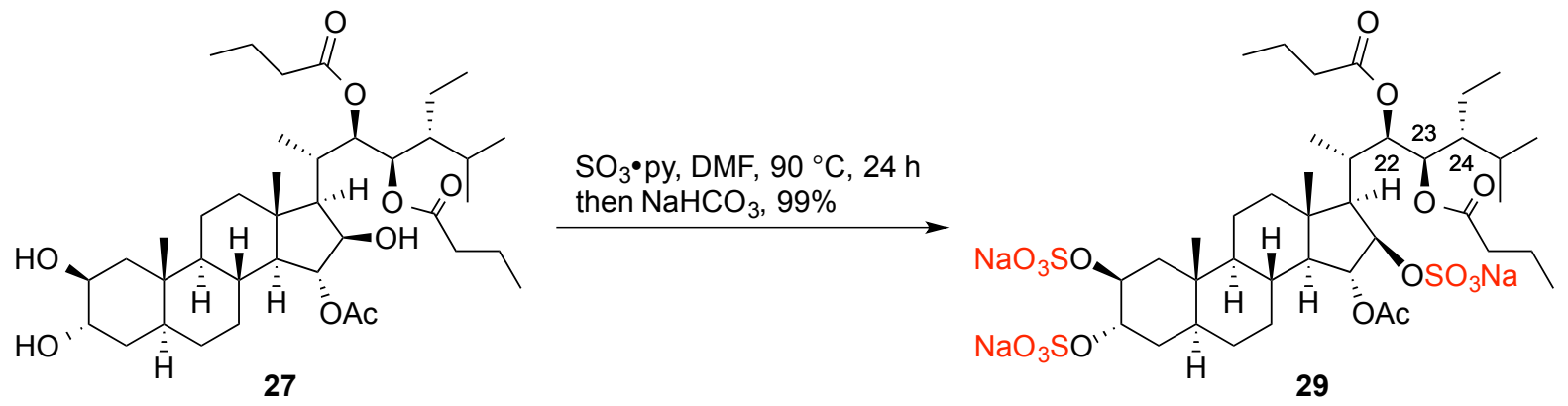

\section{Trisulfate sodium salt 29}

To a solution of $27(7 \mathrm{mg}, 0.010 \mathrm{mmol})$ in dry DMF $(1 \mathrm{~mL})$ was added $\mathrm{SO}_{3}$-pyridine complex (260 mg, $0.20 \mathrm{mmol}$ ). The reaction was stirred at $90{ }^{\circ} \mathrm{C}$ for $24 \mathrm{~h}$, quenched with water (2 $\mathrm{mL}$ ), and added solid $\mathrm{NaHCO}_{3}(400 \mathrm{mg})$. The solvents was removed under reduced pressure. We then added $\mathrm{MeOH}(2 \mathrm{~mL})$, filtered the resulting mixture, concentrated in vacuo, and purified through flash column chromatography (DCM/MeOH: 4/1) to afford 29 (11 mg, 99\%) as a white gum. $[\alpha]^{25} \mathrm{D}+19.9\left(c \mathrm{0.7}, \mathrm{CH}_{3} \mathrm{OH}\right)$; IR ( $\mathrm{KBr}$ film) $3445,2962,1732,1634,1463$, $1385,1266,1205,1068,969,590 \mathrm{~cm}^{-1} ;{ }^{1} \mathrm{H}$ NMR $\left(400 \mathrm{MHz}, \mathrm{CD}_{3} \mathrm{OH}\right) \delta 5.56(\mathrm{~d}, 1 \mathrm{H}, J=6.0$ Hz), $5.47(\mathrm{dd}, 1 \mathrm{H}, J=9.0,1.8 \mathrm{~Hz}), 5.40(\mathrm{~d}, 1 \mathrm{H}, J=8.4,2.4 \mathrm{~Hz}), 4.90(\mathrm{dd}, 1 \mathrm{H}, J=7.2,1.4$ $\mathrm{Hz}), 4.66-4.75(\mathrm{~m}, 2 \mathrm{H}), 3.32(\mathrm{~s}, 1 \mathrm{H}), 3.85-3.88(\mathrm{~m}, 2 \mathrm{H}), 3.12(\mathrm{~s}, 1 \mathrm{H}), 2.12-2.32(\mathrm{~m}, 6 \mathrm{H})$, $2.04(\mathrm{~s}, 3 \mathrm{H}), 0.86(\mathrm{~d}, 3 \mathrm{H}, J=6.8 \mathrm{~Hz}) ;{ }^{13} \mathrm{C} \mathrm{NMR}\left(100 \mathrm{MHz}, \mathrm{CD}_{3} \mathrm{OH}\right) \delta 173.5,173.0,172.2$, 85.0, 81.9, 75.5, 75.2, 75.0, 74.62, 58.8, 54.5, 45.5, 43.6, 40.1, 38.3, 37.7, 35.8, 35.8, 34.7, $33.8,31.1,30.0,29.0,27.3,25.7,22.4,20.6,20.0,18.1,17.9,17.1,16.9,13.1,12.7,12.6$, 12.5, 11.7, 11.6; LRMS-ESI $(\mathrm{m} / \mathrm{z}): 961.3\left(\left[\mathrm{M}-\mathrm{Na}^{+}\right]^{-}\right)$; HRMS-ESI $(\mathrm{m} / \mathrm{z}):\left[\mathrm{M}-\mathrm{Na}^{+}\right]^{-}$calcd for $\mathrm{C}_{39} \mathrm{H}_{63} \mathrm{Na}_{3} \mathrm{O}_{18} \mathrm{~S}_{3}$ 961.2977, found 961.2969. 


\section{NMR Spectra}

${ }^{1} \mathrm{H}$ NMR spectra of diol S1 (300 $\left.\mathrm{MHz}, \mathrm{CDCl}_{3}\right)$

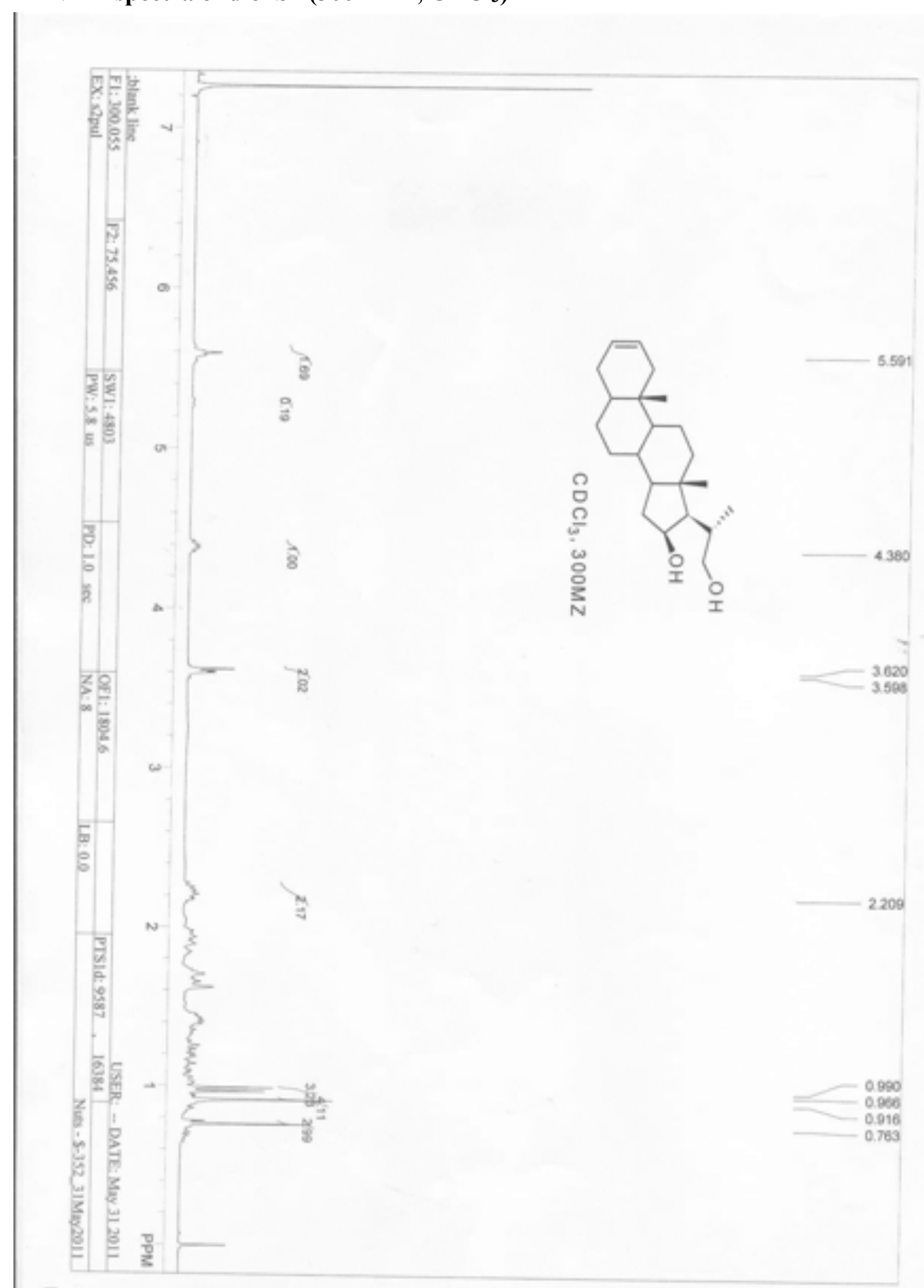


${ }^{13} \mathrm{C}$ NMR spectra of diol S1 (100 $\left.\mathrm{MHz}, \mathrm{CDCl}_{3}\right)$

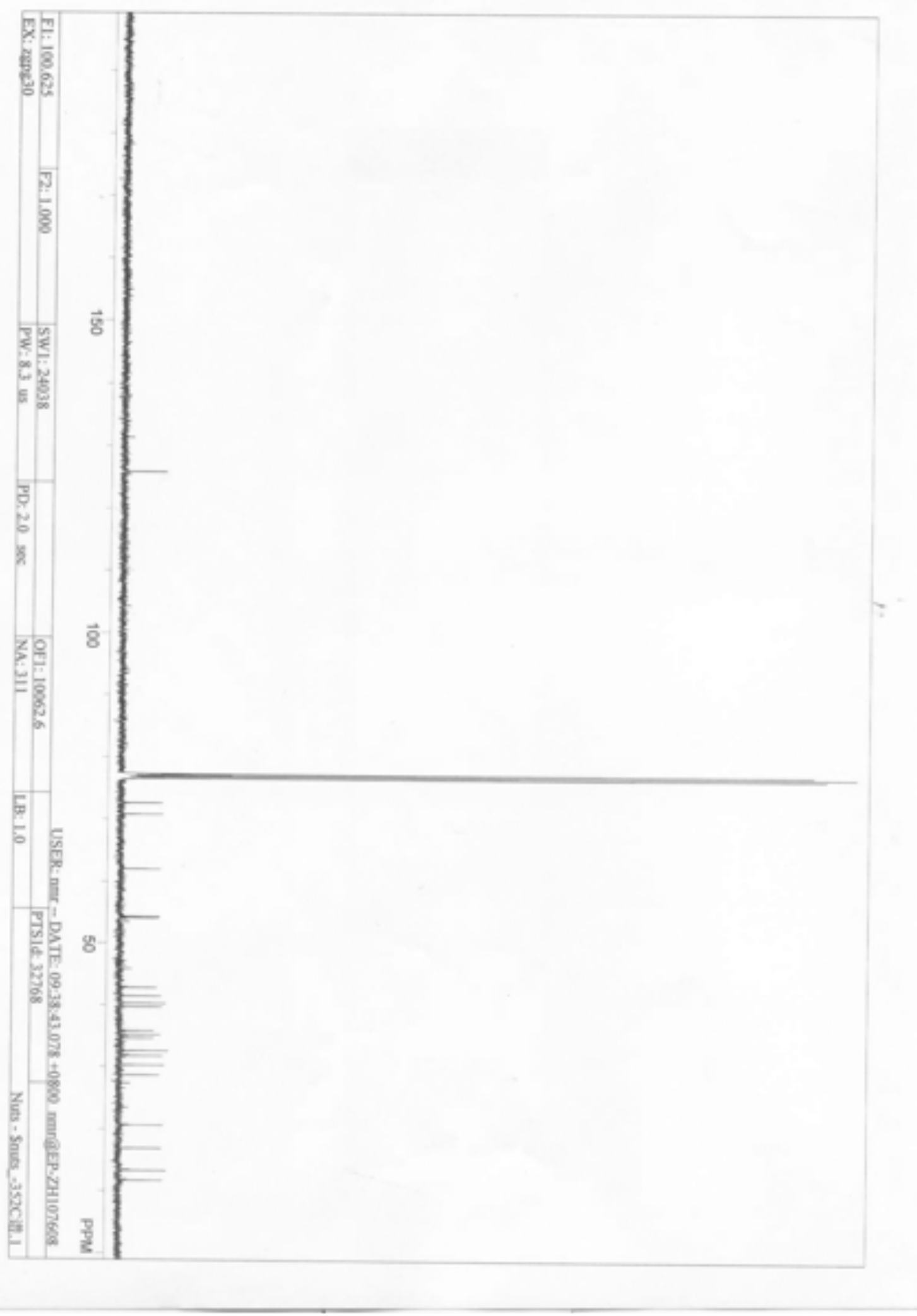


${ }^{1} \mathrm{H}$ NMR spectra of mono-TBS ether $8\left(300 \mathrm{MHz}, \mathrm{CDCl}_{3}\right)$

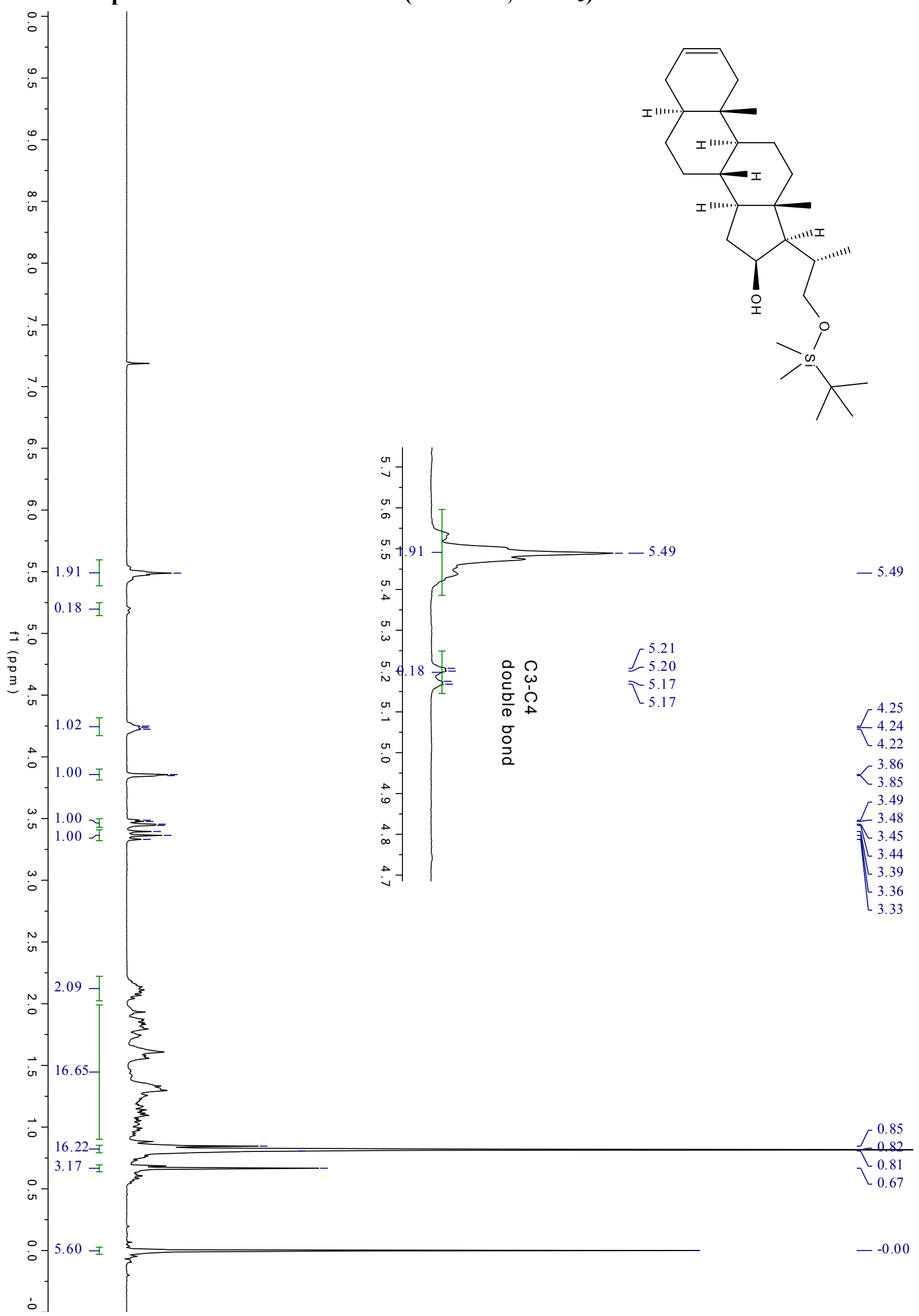


${ }^{13} \mathrm{C}$ NMR spectra of mono-TBS ether $8\left(100 \mathrm{MHz}, \mathrm{CDCl}_{3}\right)$

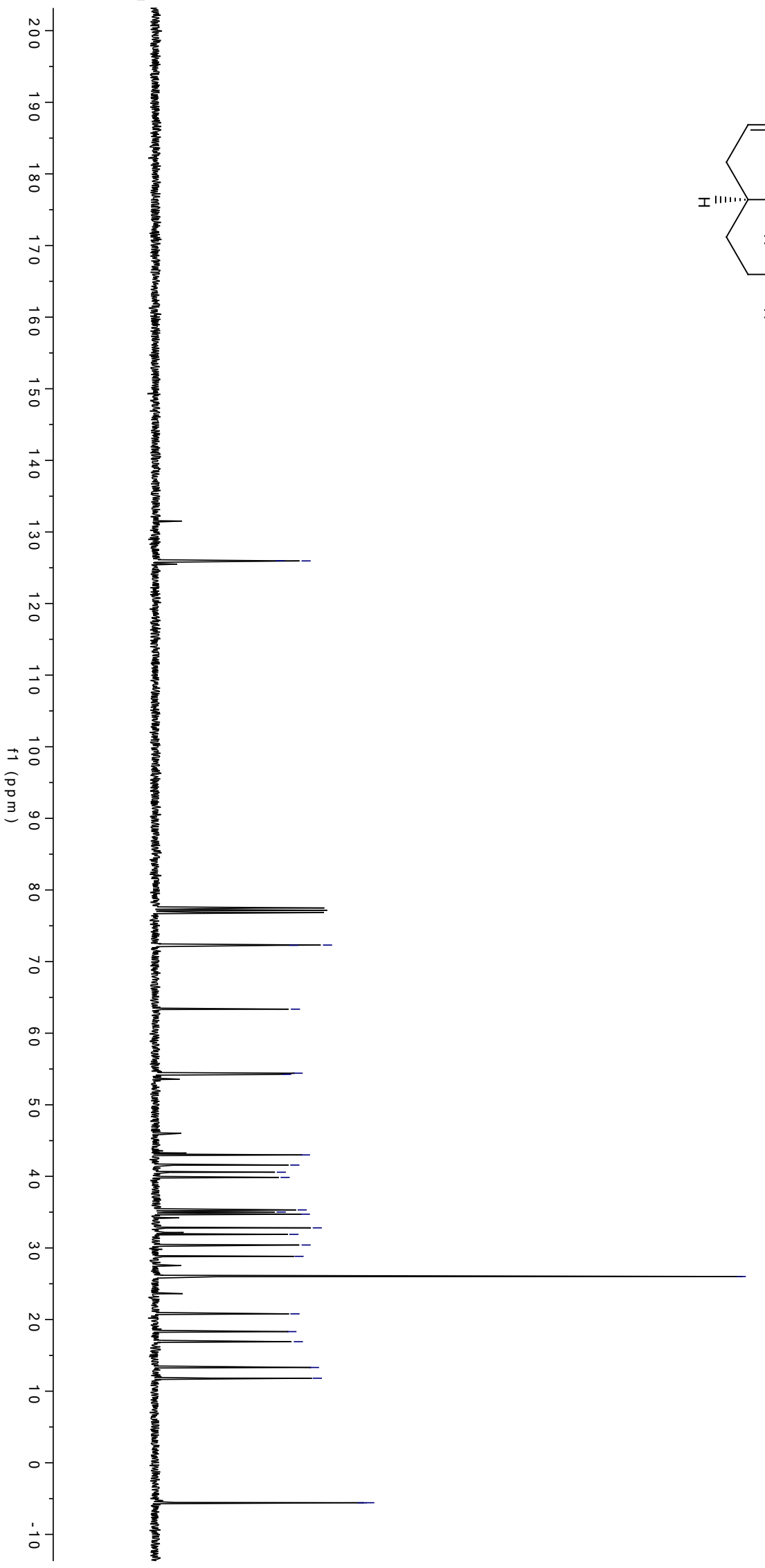


${ }^{1} \mathrm{H}$ NMR spectra of compound $\mathrm{S} 2\left(300 \mathrm{MHz}, \mathrm{CDCl}_{3}\right)$

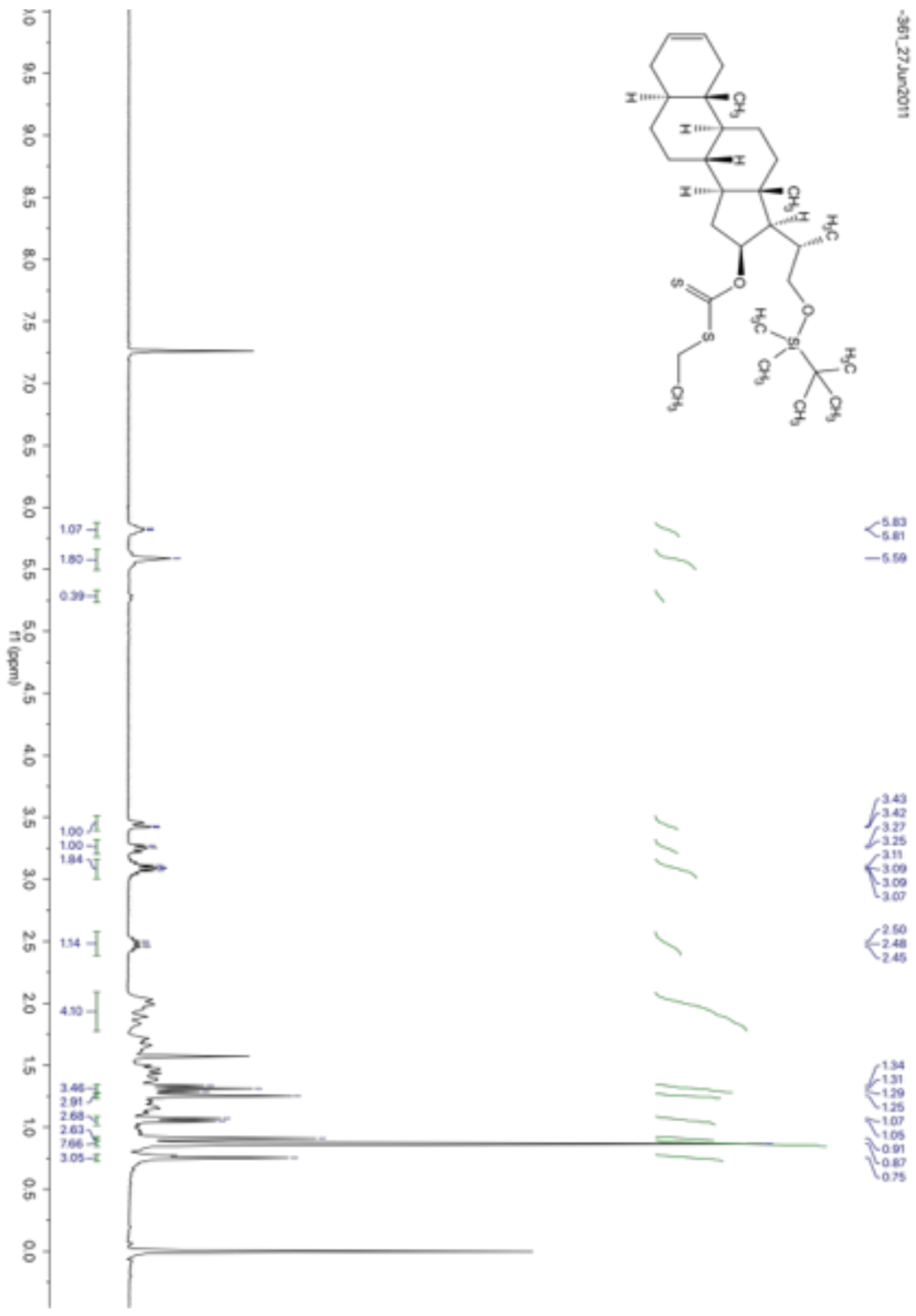


${ }^{13} \mathrm{C}$ NMR spectra of compound S2 (100 $\left.\mathrm{MHz} \mathrm{CDCl}_{3}\right)$

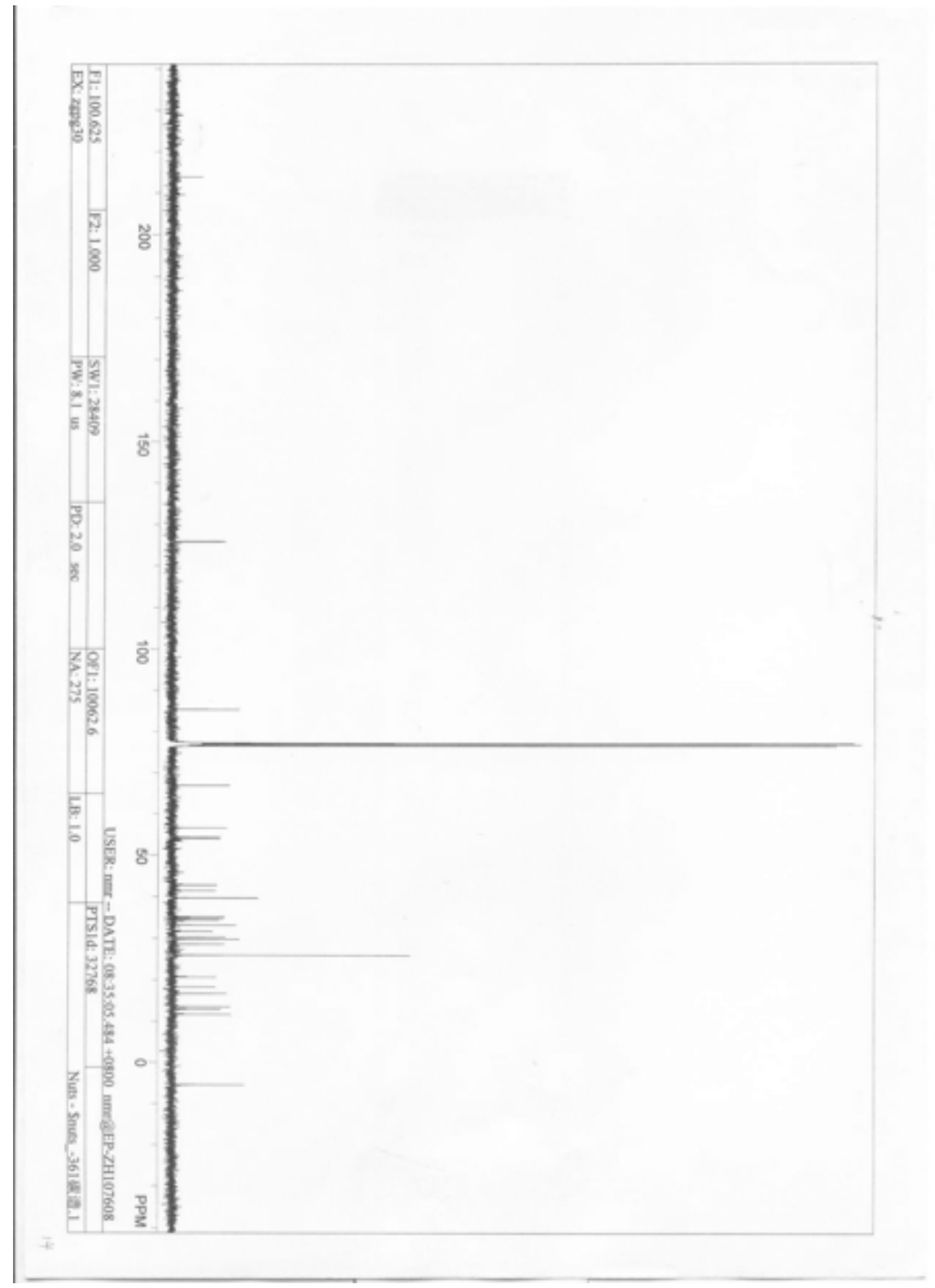


${ }^{1} \mathrm{H}$ NMR spectra of compound $9\left(300 \mathrm{MHz}, \mathrm{CDCl}_{3}\right)$

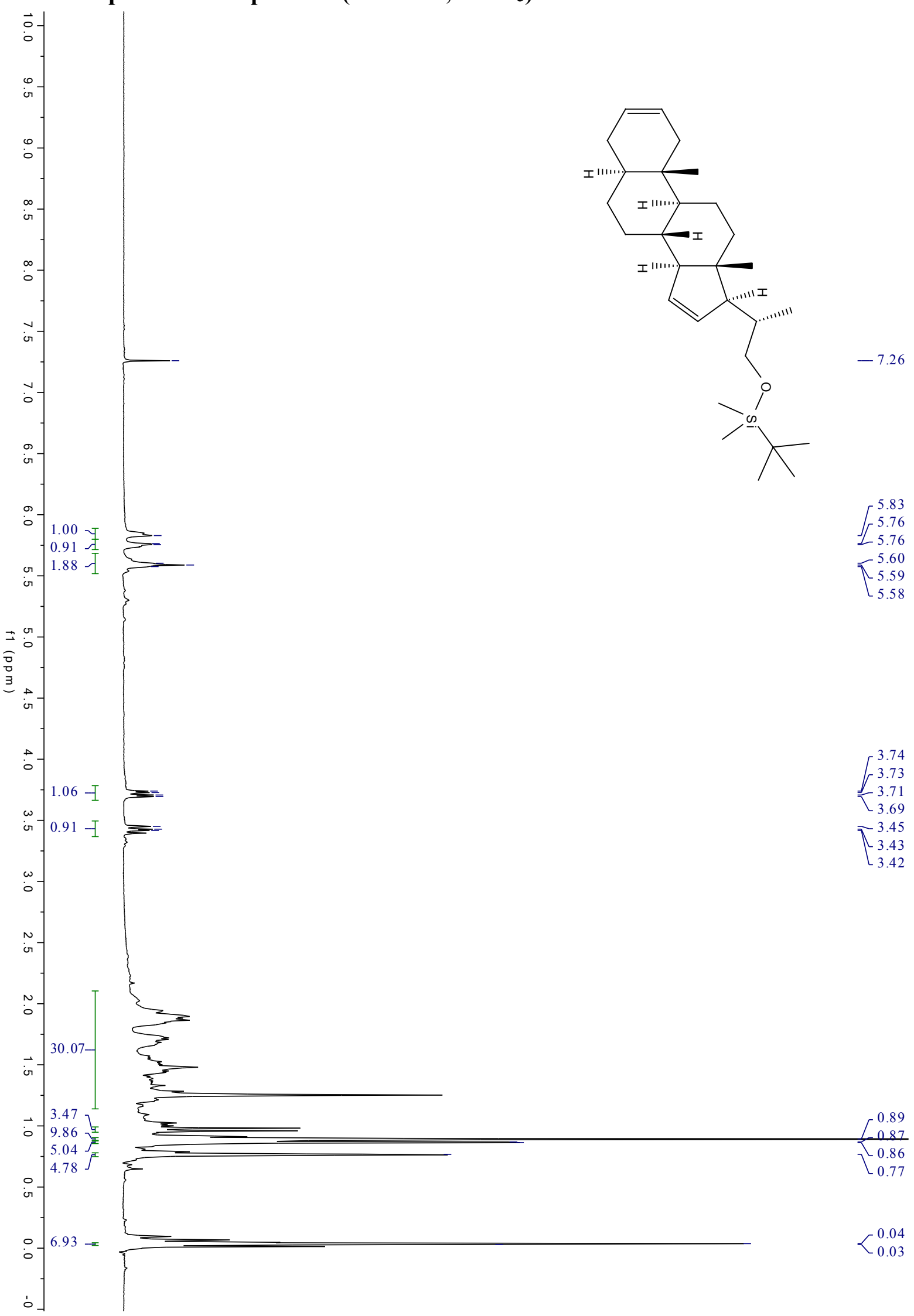


${ }^{13} \mathrm{C}$ NMR spectra of compound $9\left(100 \mathrm{MHz}, \mathrm{CDCl}_{3}\right)$

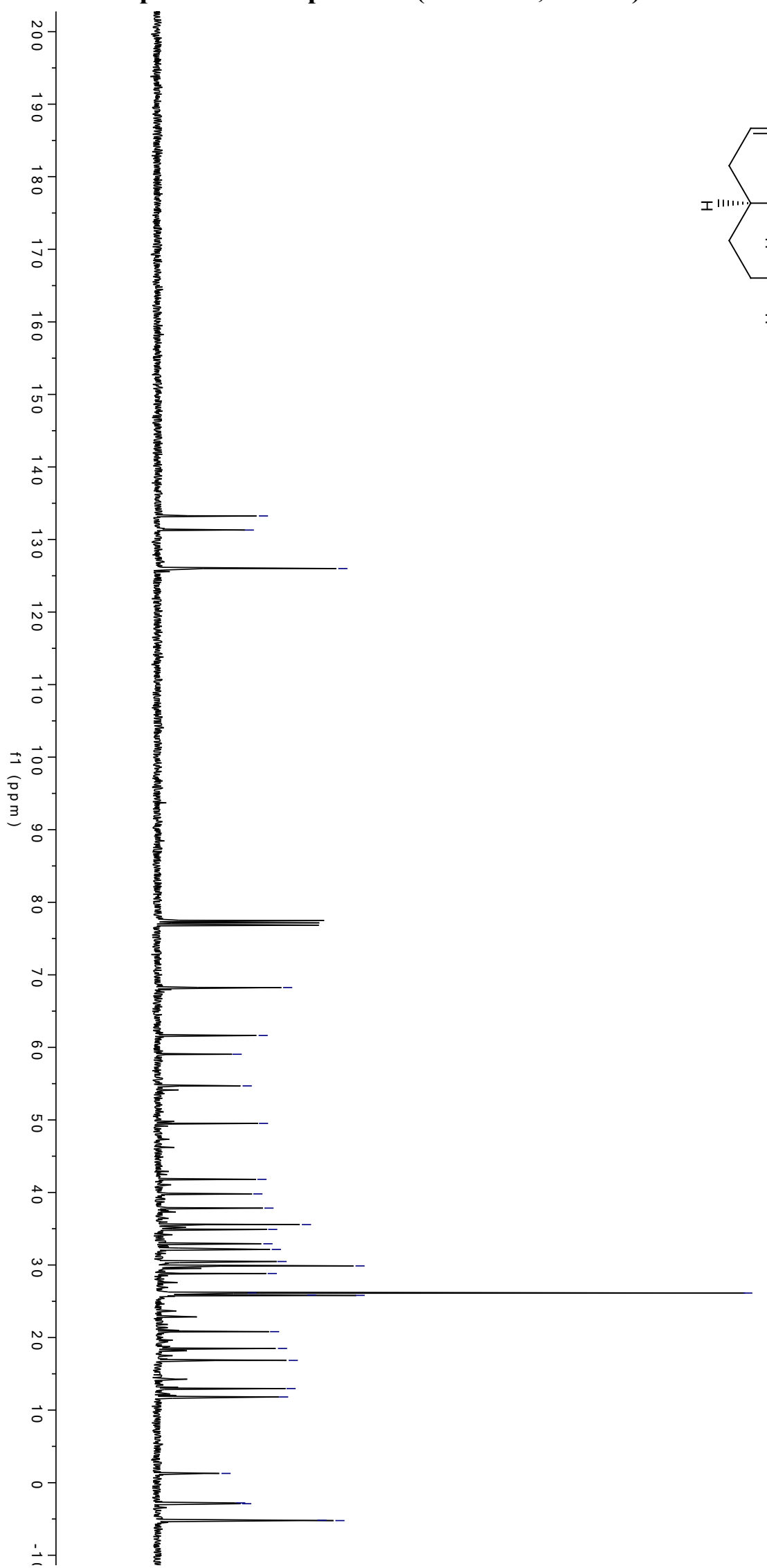

$\simeq 133.25$
$\simeq 131.31$
-126.00

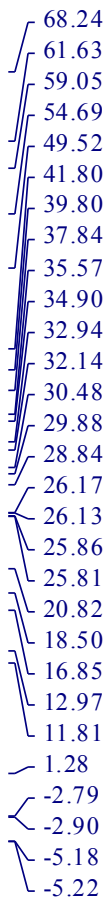


${ }^{1} \mathrm{H}$ NMR spectra of compound $\mathrm{S3}\left(300 \mathrm{MHz}, \mathrm{CDCl}_{3}\right)$

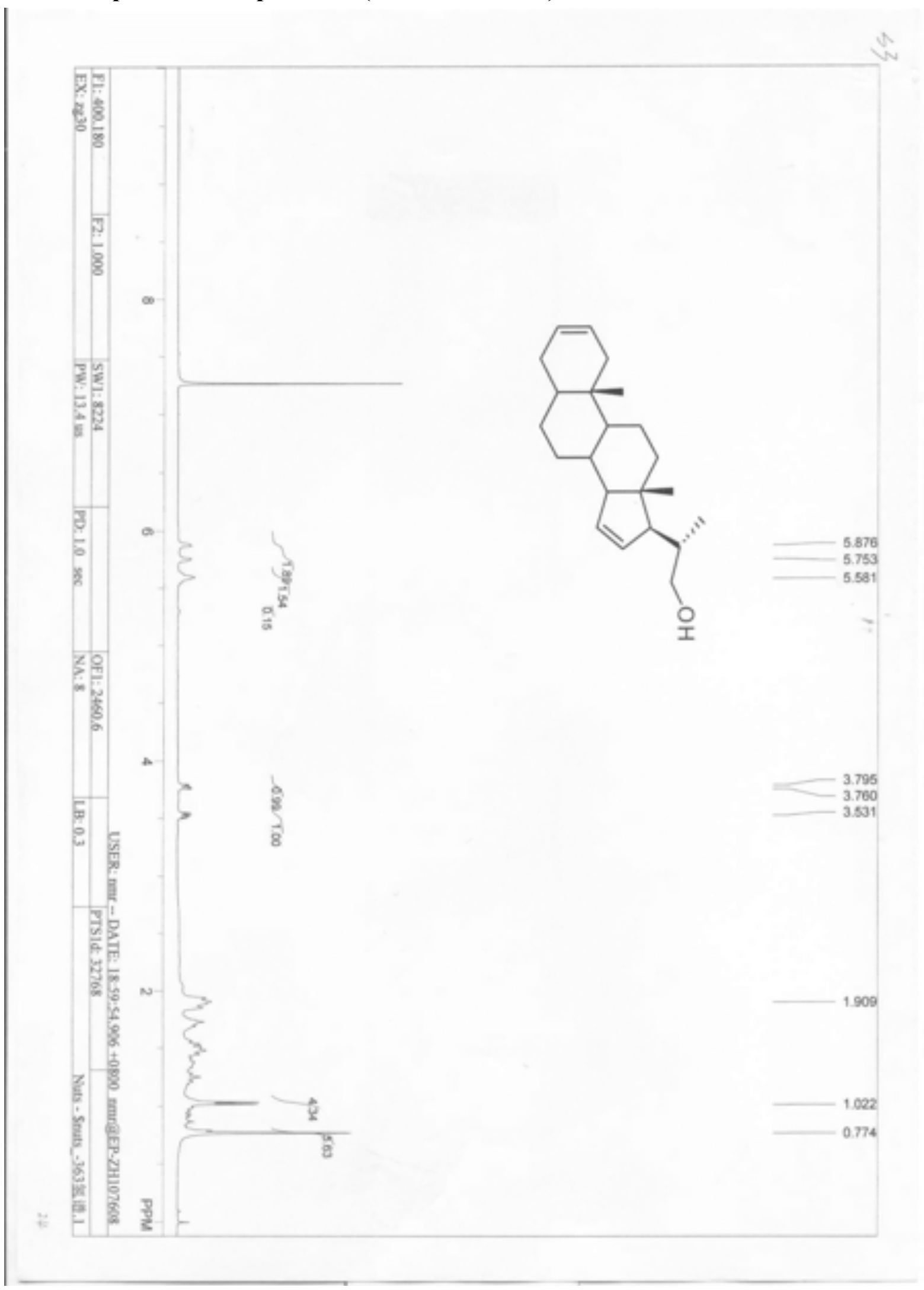


${ }^{13} \mathrm{C}$ NMR spectra of compound $\mathrm{S3}\left(100 \mathrm{MHz}, \mathrm{CDCl}_{3}\right)$

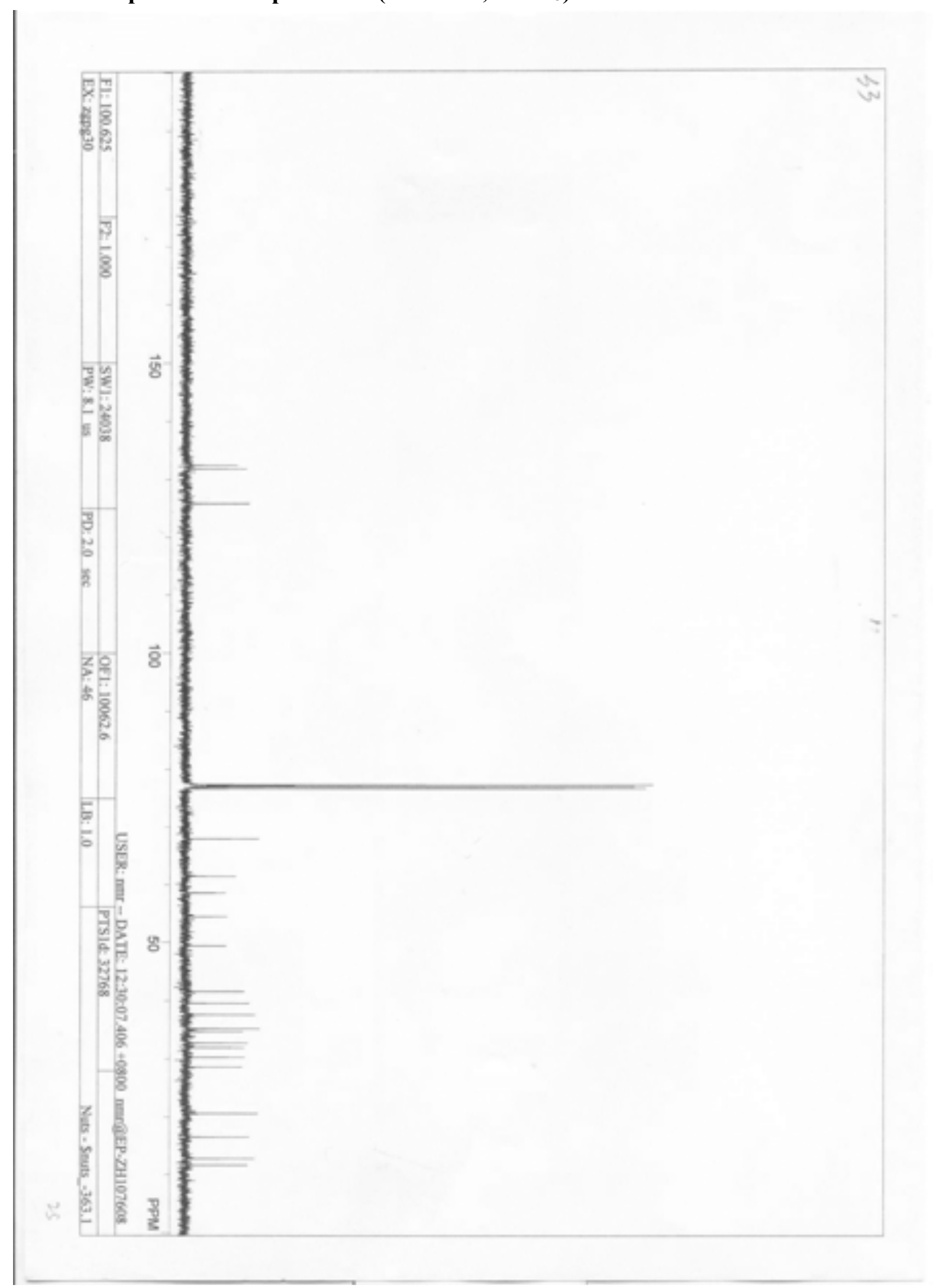


${ }^{1} \mathrm{H}$ NMR spectra of known compound 6 (300 $\left.\mathrm{MHz}, \mathrm{CDCl}_{3}\right)$

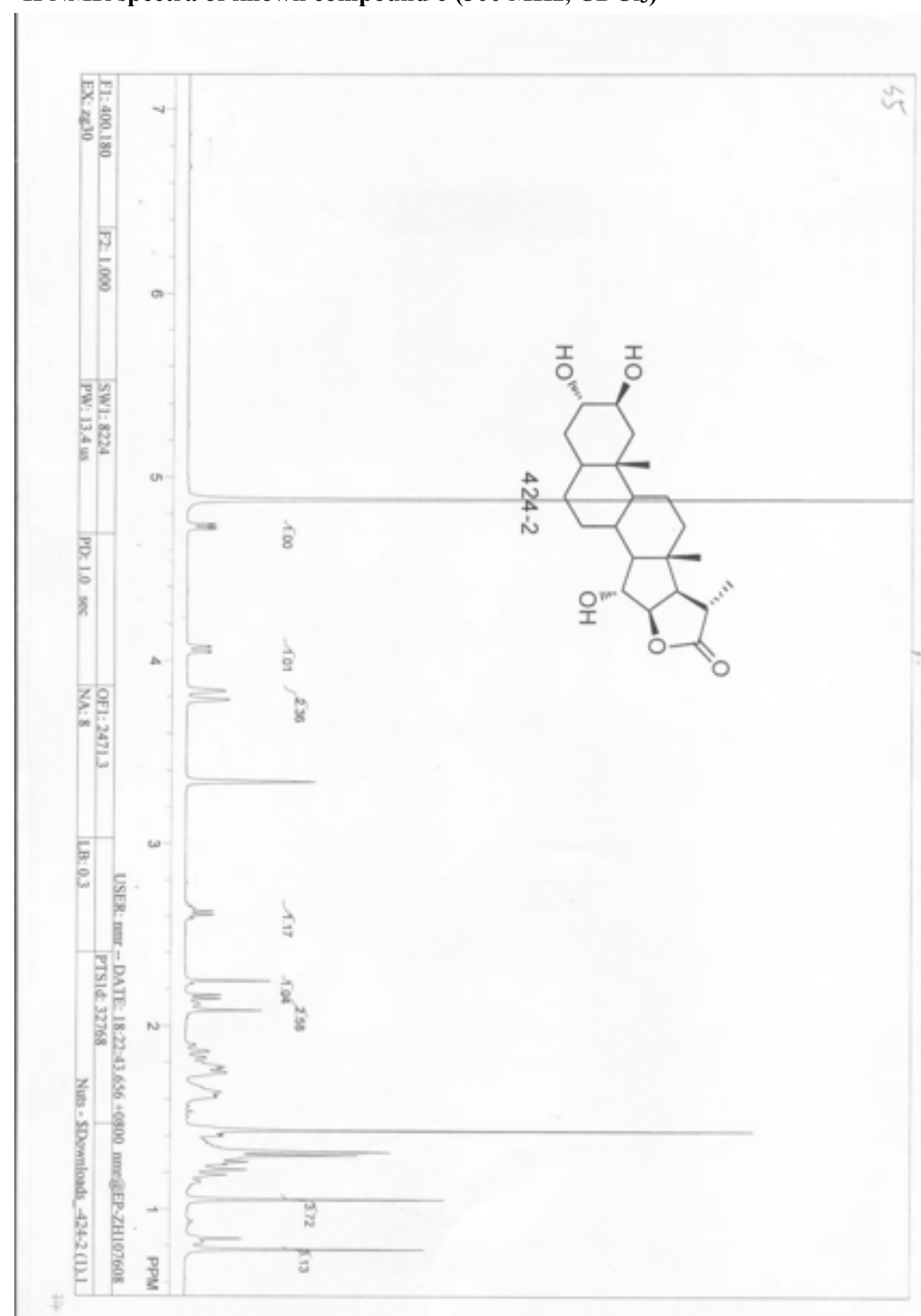


${ }^{1} \mathrm{H}$ NMR spectra of compound $11\left(300 \mathrm{MHz}, \mathrm{CDCl}_{3}\right)$

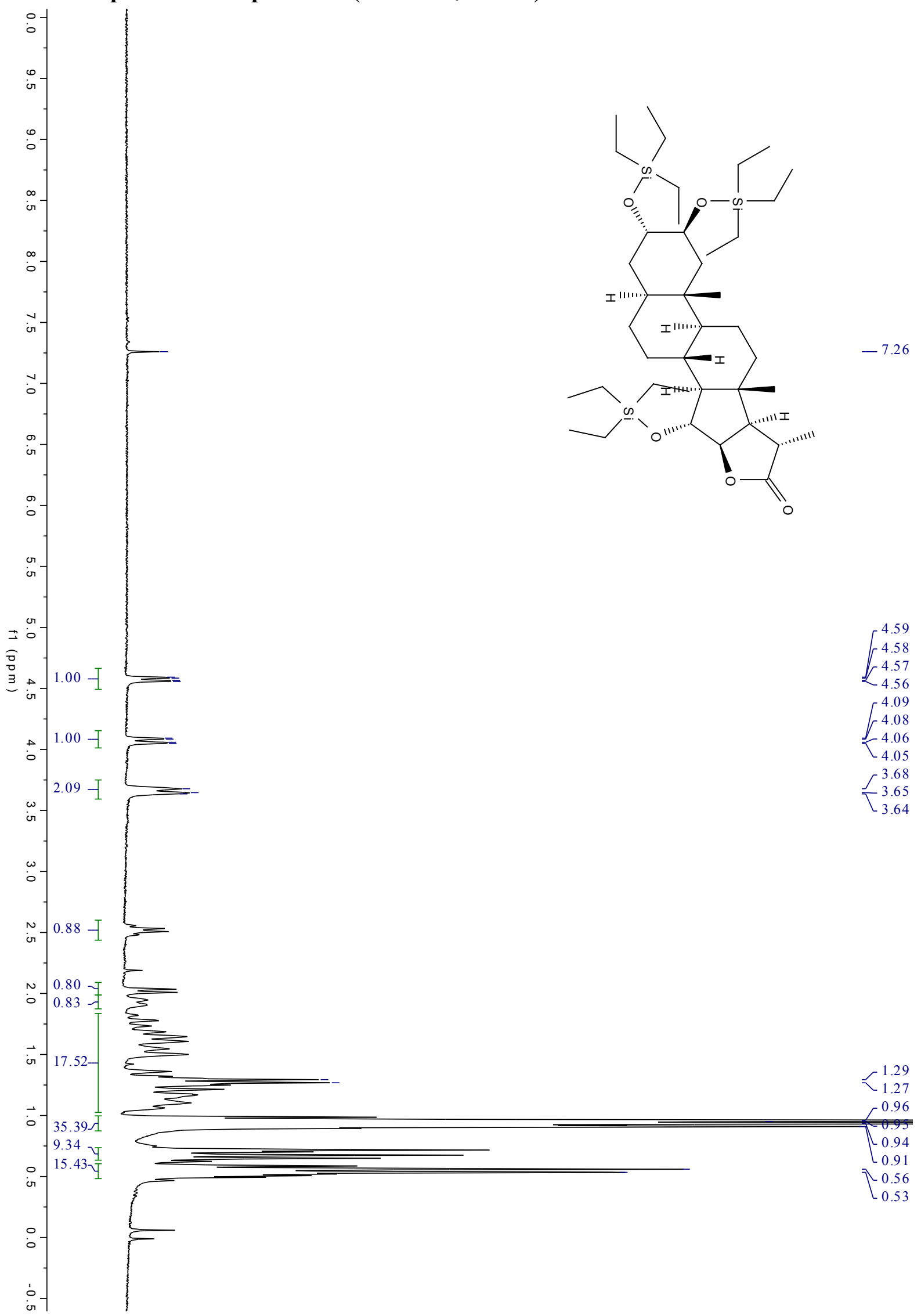


${ }^{13} \mathrm{C}$ NMR spectra of compound $11\left(100 \mathrm{MHz}, \mathrm{CDCl}_{3}\right)$

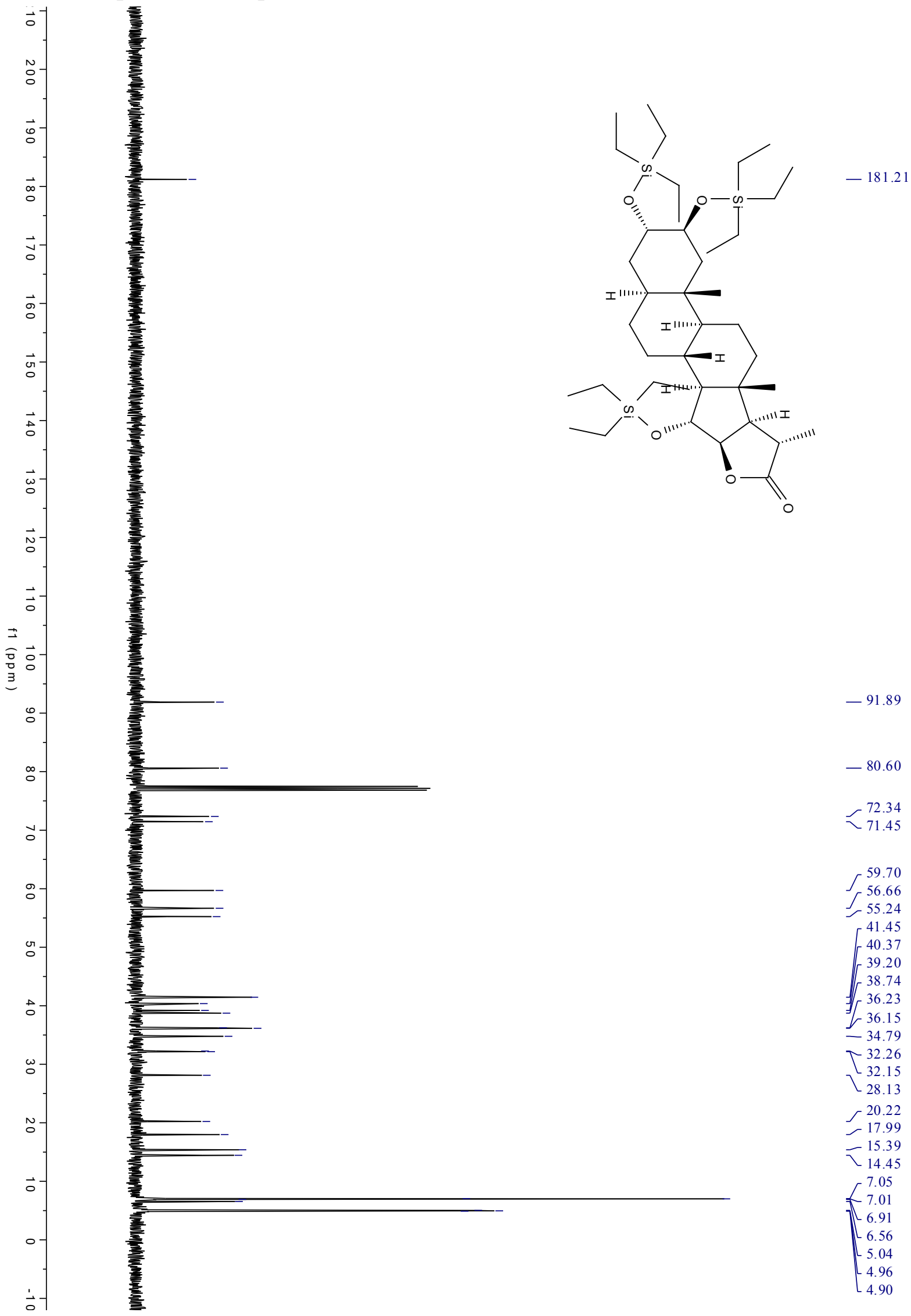


${ }^{1} \mathrm{H}$ NMR spectra of dibromide $12\left(400 \mathrm{MHz}, \mathrm{CDCl}_{3}\right)$

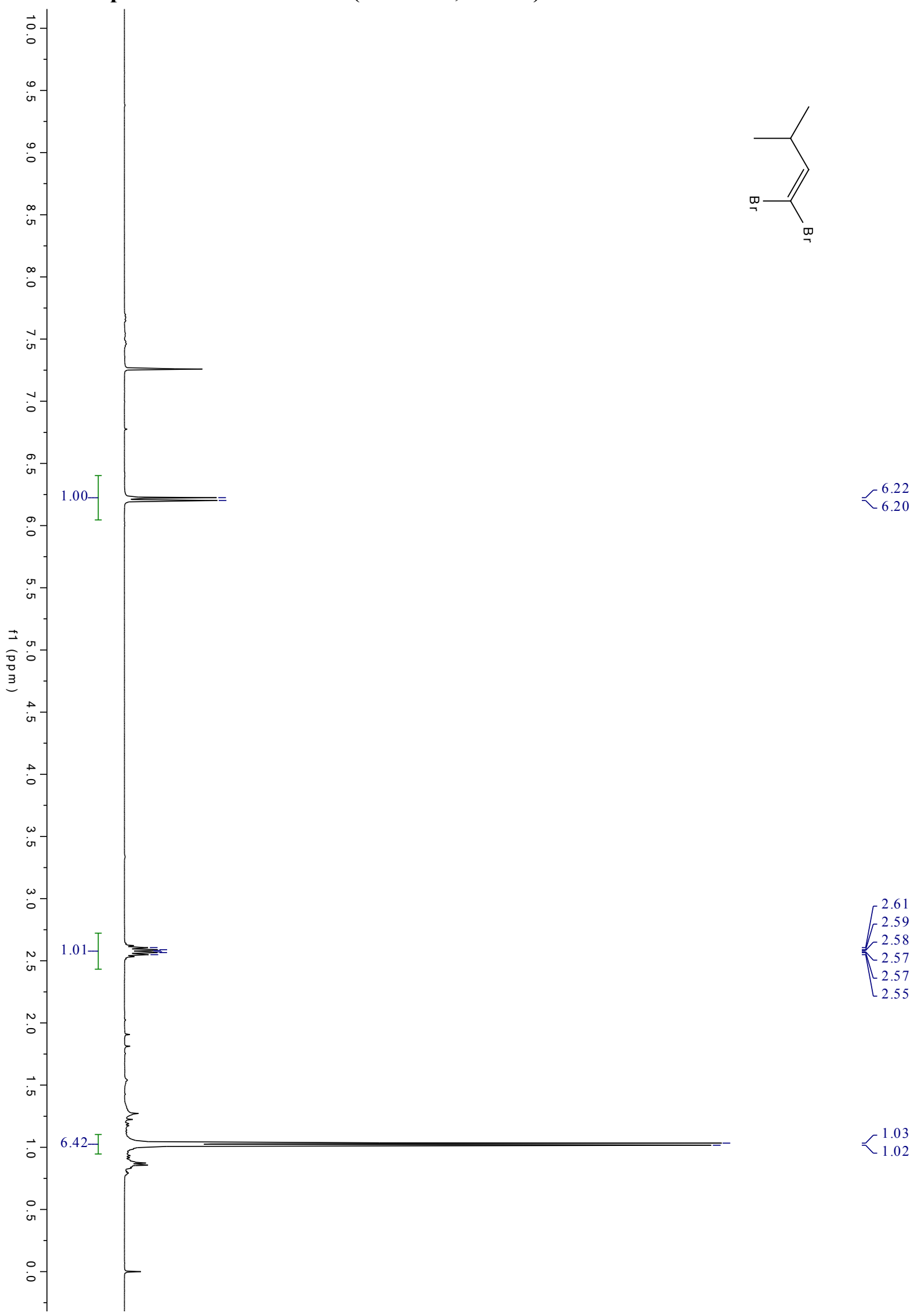




\section{${ }^{1} \mathrm{H}$ NMR spectra of compound $13\left(400 \mathrm{MHz}, \mathrm{CDCl}_{3}\right)$}

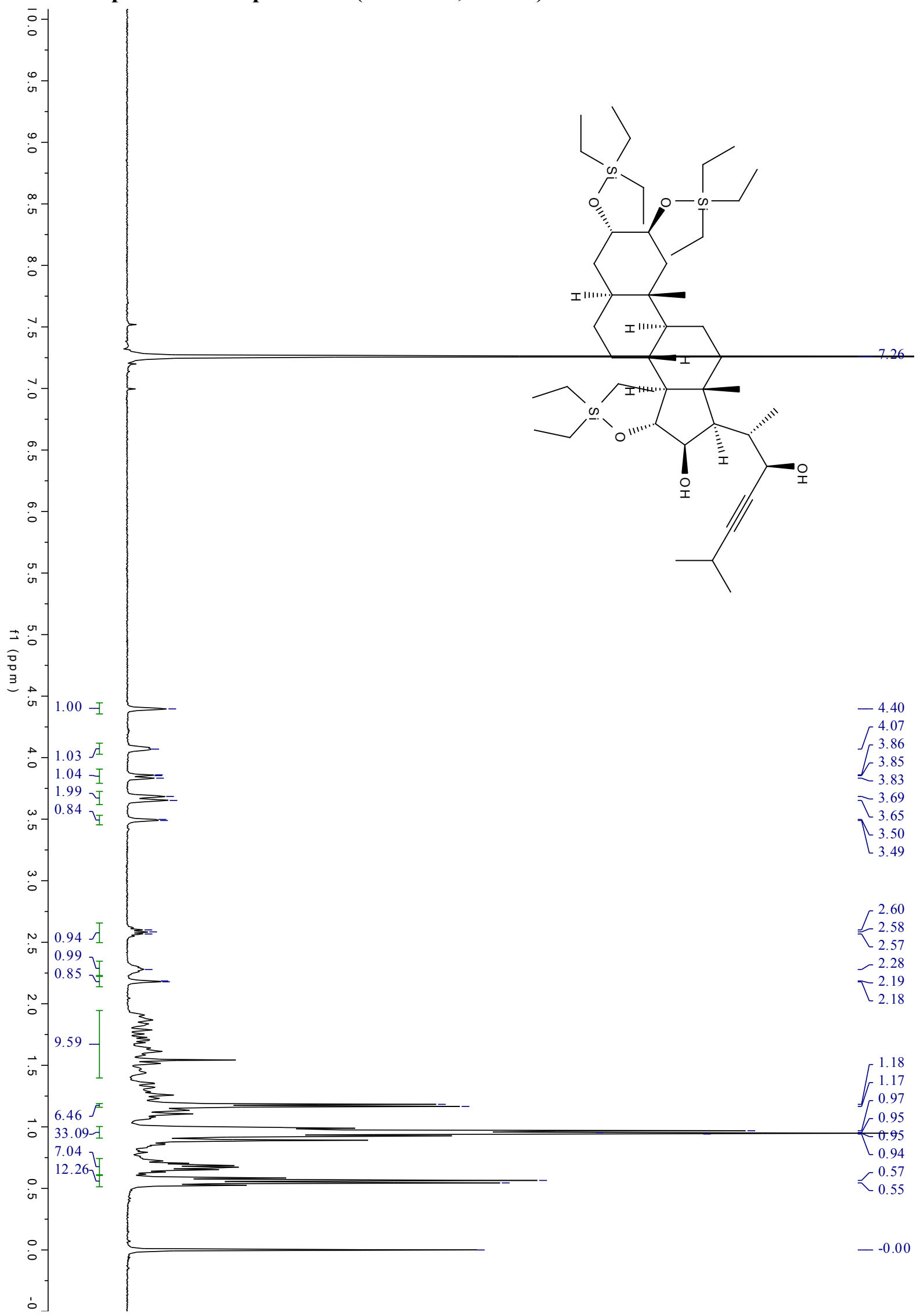


${ }^{13} \mathrm{C}$ NMR spectra of compound $13\left(100 \mathrm{MHz}, \mathrm{CDCl}_{3}\right)$

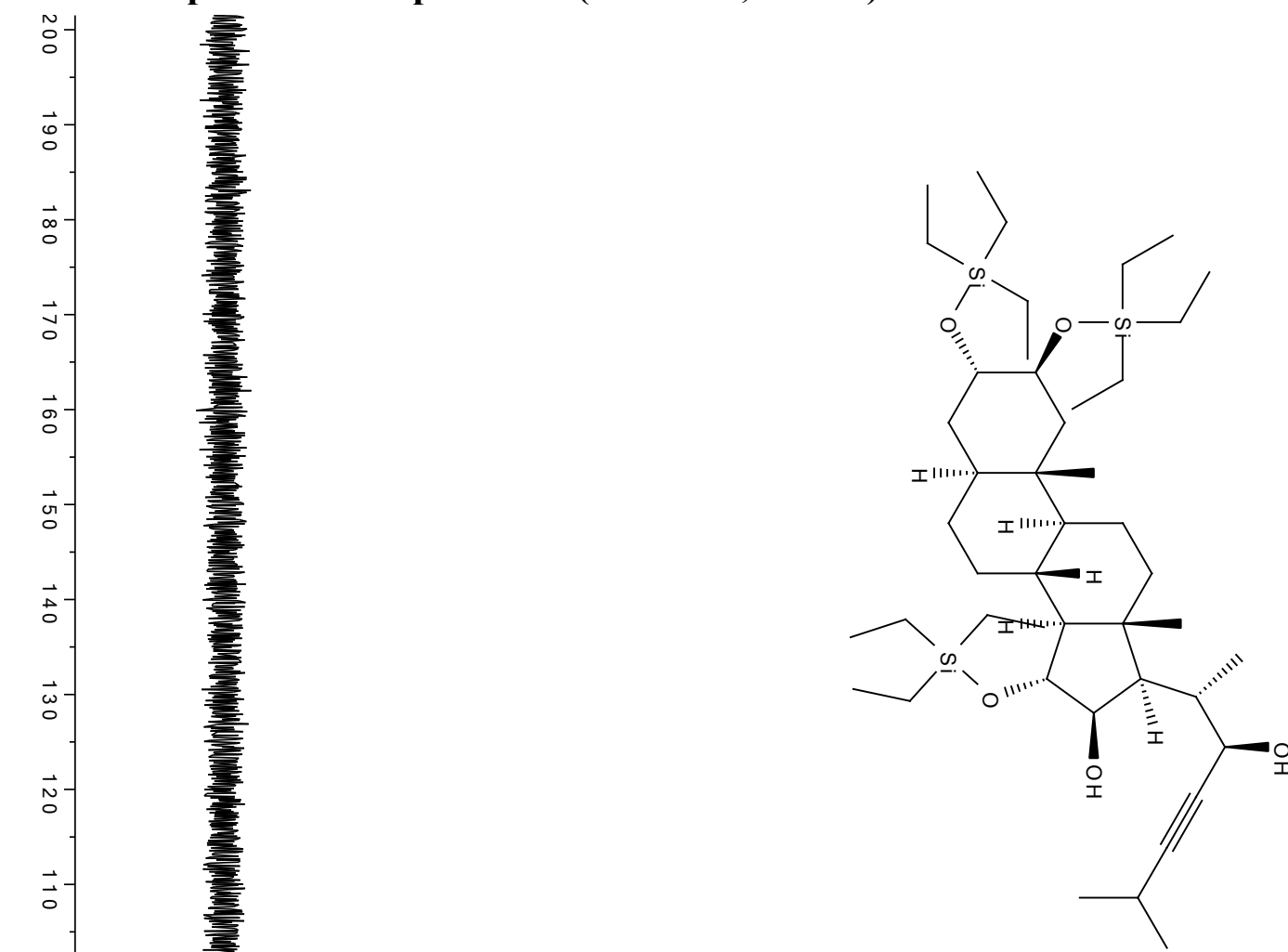


${ }^{1} \mathrm{H}$ NMR spectra of compound $5\left(300 \mathrm{MHz}, \mathrm{CDCl}_{3}\right)$

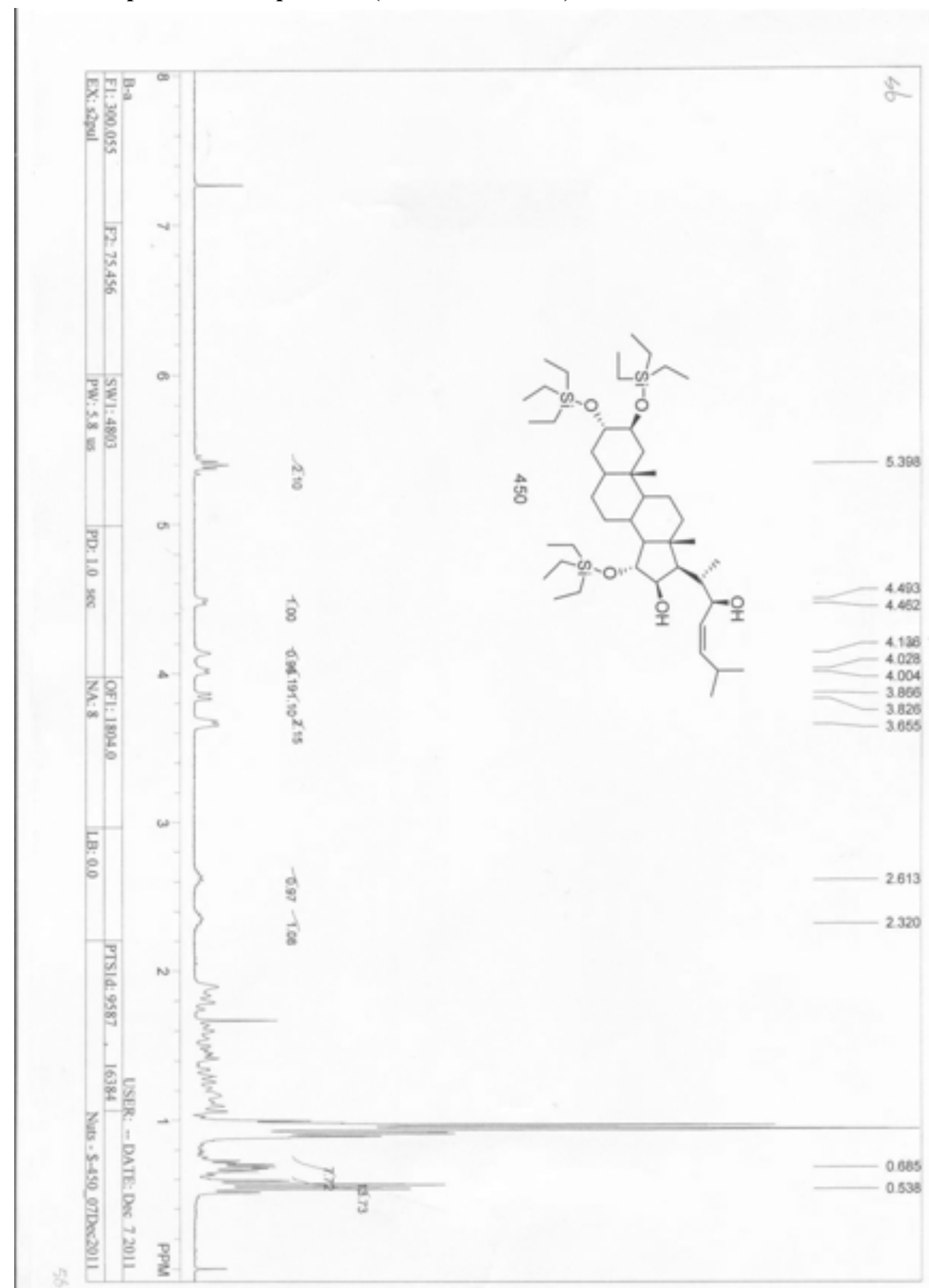


${ }^{13} \mathrm{C}$ NMR spectra of compound $5\left(100 \mathrm{MHz}, \mathrm{CDCl}_{3}\right)$

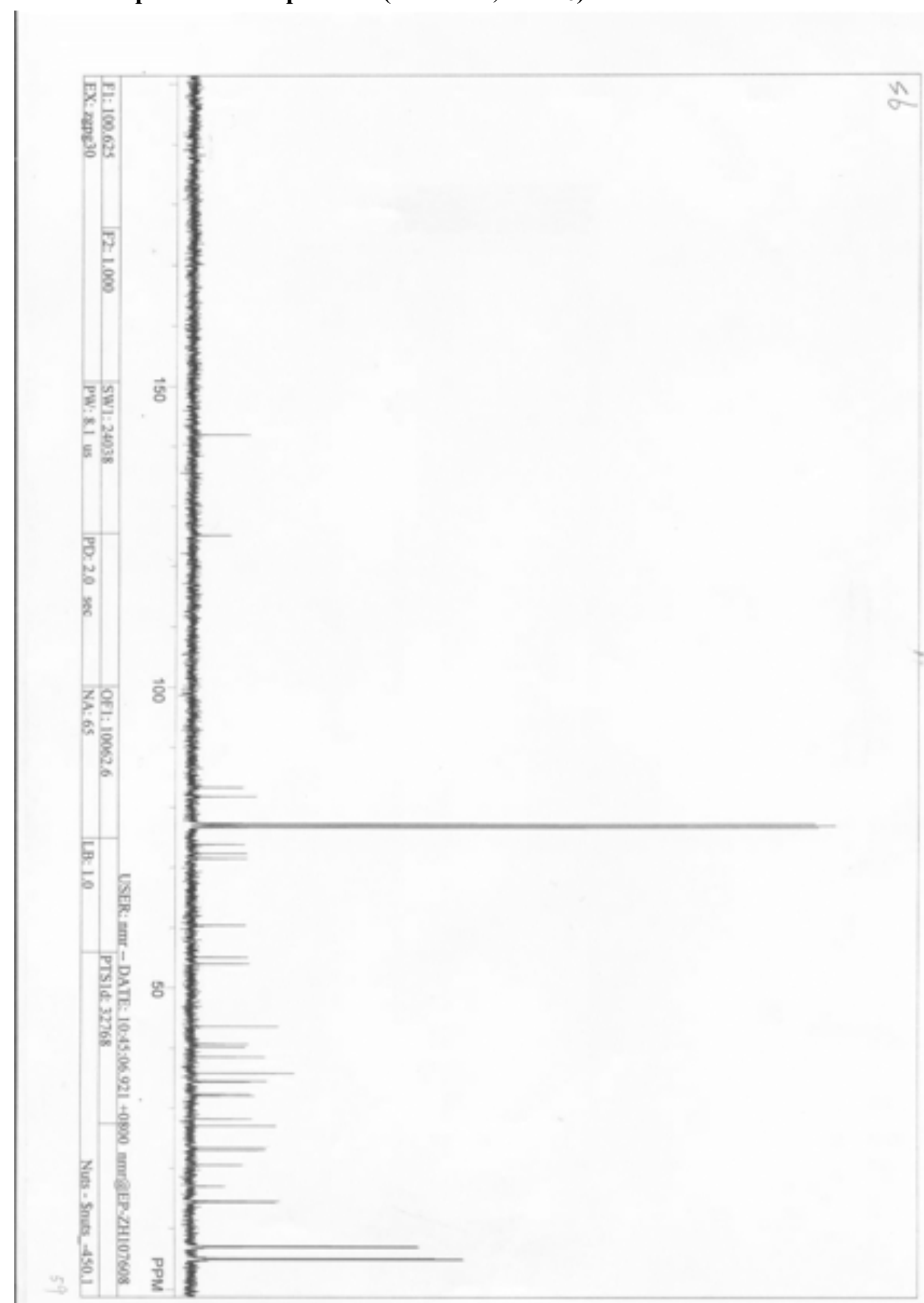


${ }^{1} \mathrm{H}$ NMR spectra of compound $14\left(400 \mathrm{MHz}, \mathrm{CDCl}_{3}\right)$

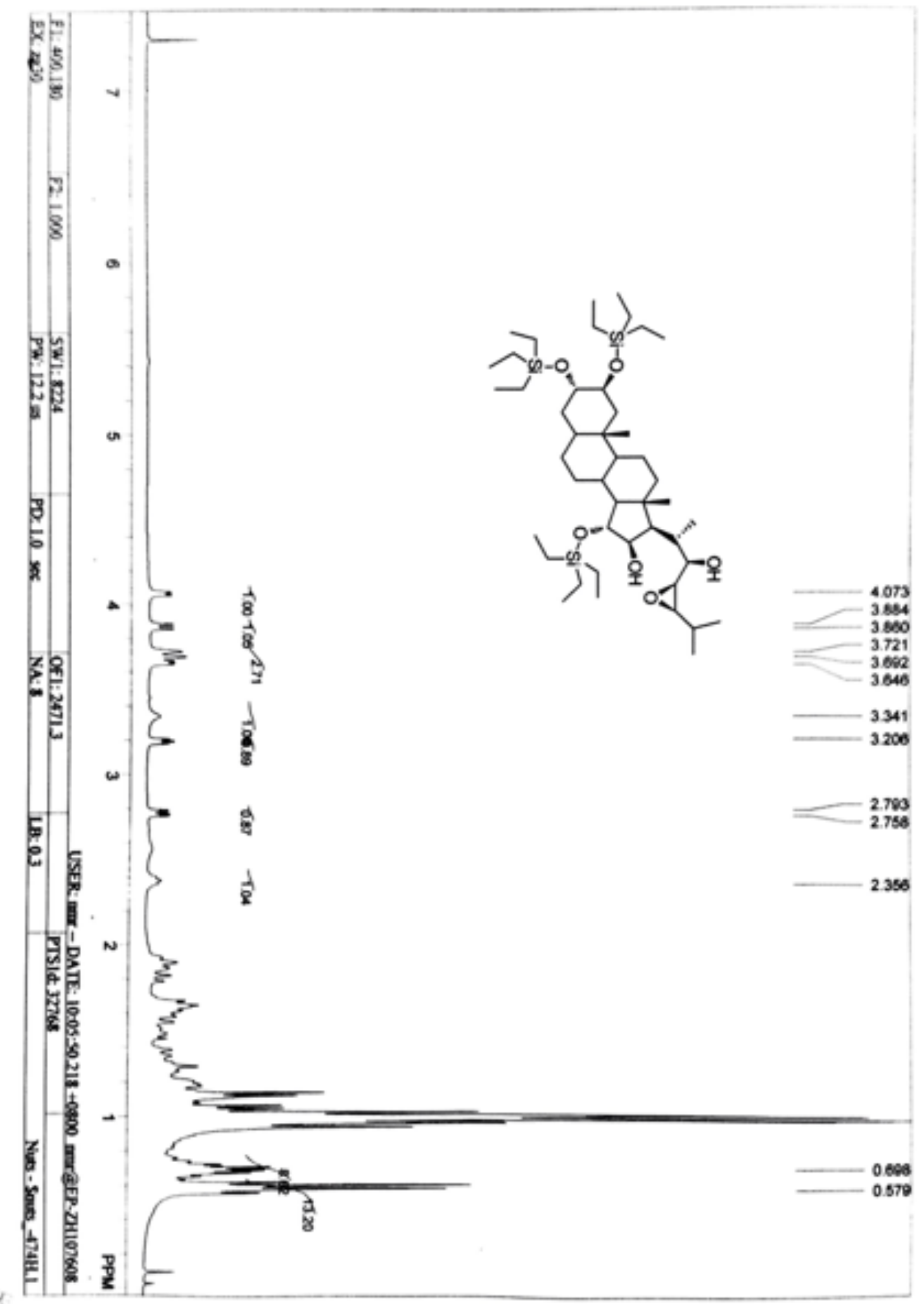


${ }^{13} \mathrm{C}$ NMR spectra of compound $14\left(100 \mathrm{MHz}, \mathrm{CDCl}_{3}\right)$

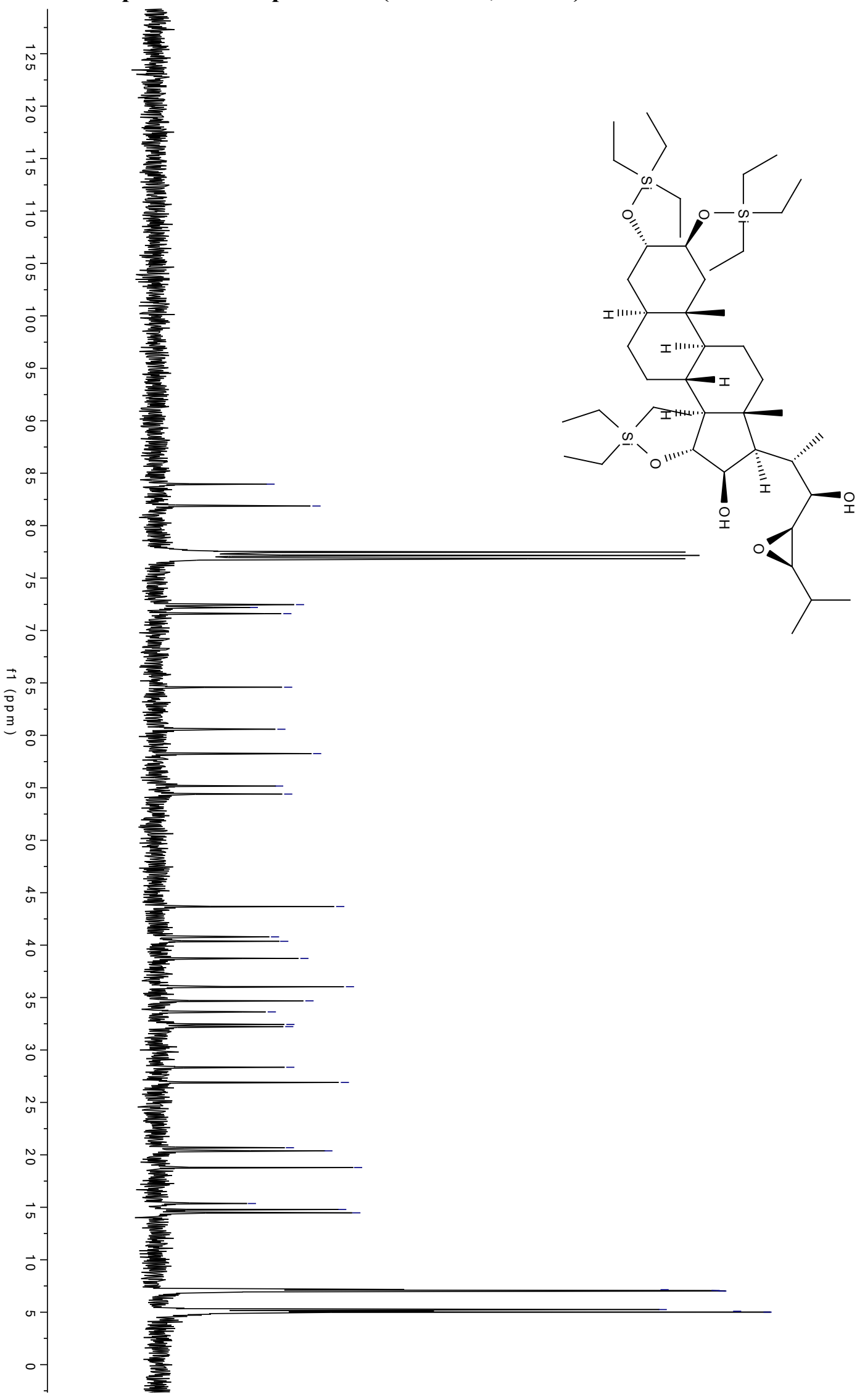

$-83.96$

$-81.87$

$-64.59$

$-60.59$

$-58.26$

55.17

54.4

43.68
-40.79

$\int_{-}^{40.36}$

38.73

36.04

34.69

33.63

$-32.43$

32.23

$-28.35$

$-26.91$

$\tau^{20.68}$

$-20.38$

$-18.79$

15.36

$-14.79$

$\bigcup_{14.47}$

7.15

7.15
7.06

$\prod_{5.25}^{5.09}$

5.00 
${ }^{13} \mathrm{C}$ NMR DEPT135 spectra of compound $14\left(100 \mathrm{MHz}, \mathrm{CDCl}_{3}\right)$

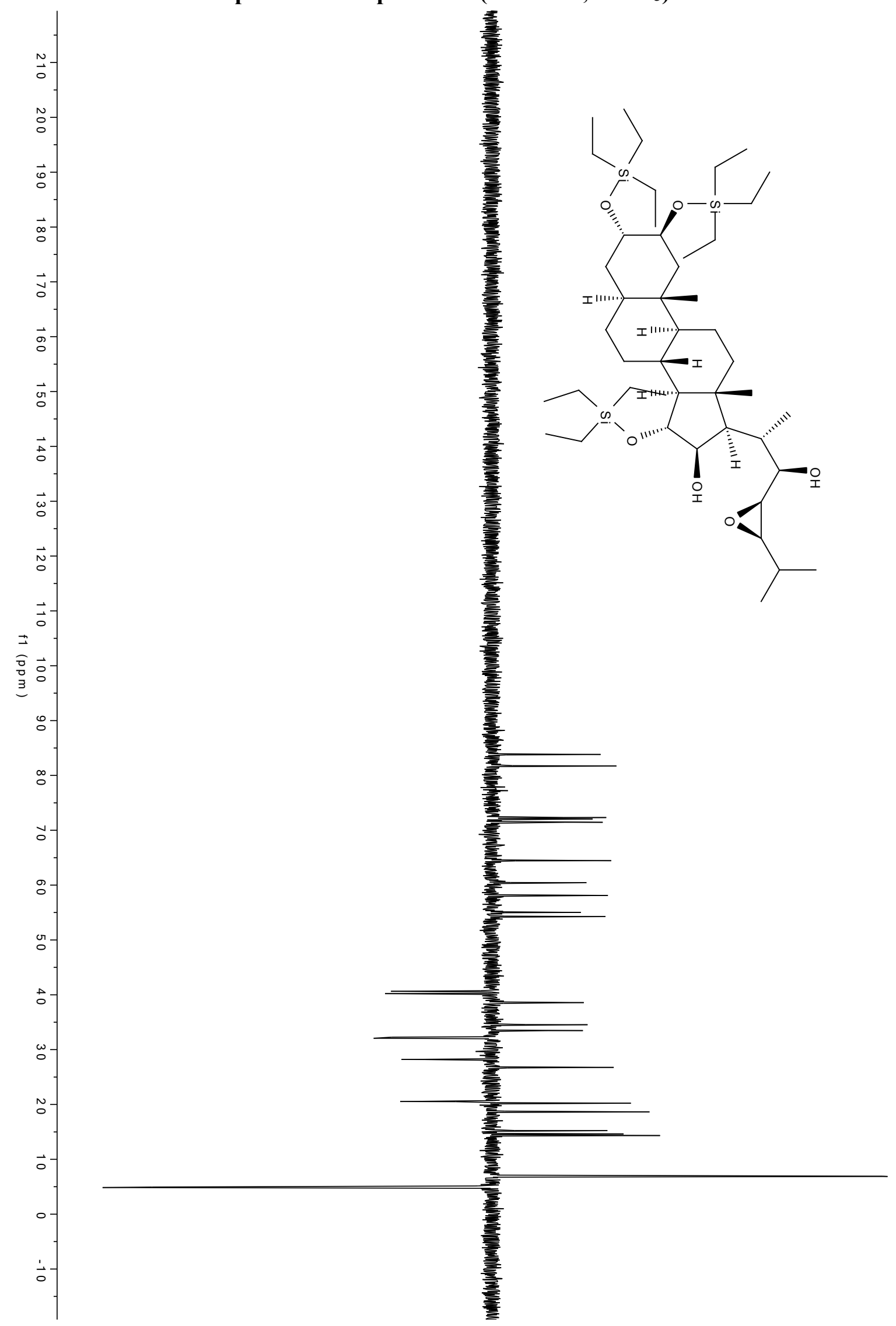


${ }^{1} \mathrm{H}$ NMR spectra of compound $15\left(400 \mathrm{MHz}, \mathrm{CDCl}_{3}\right)$

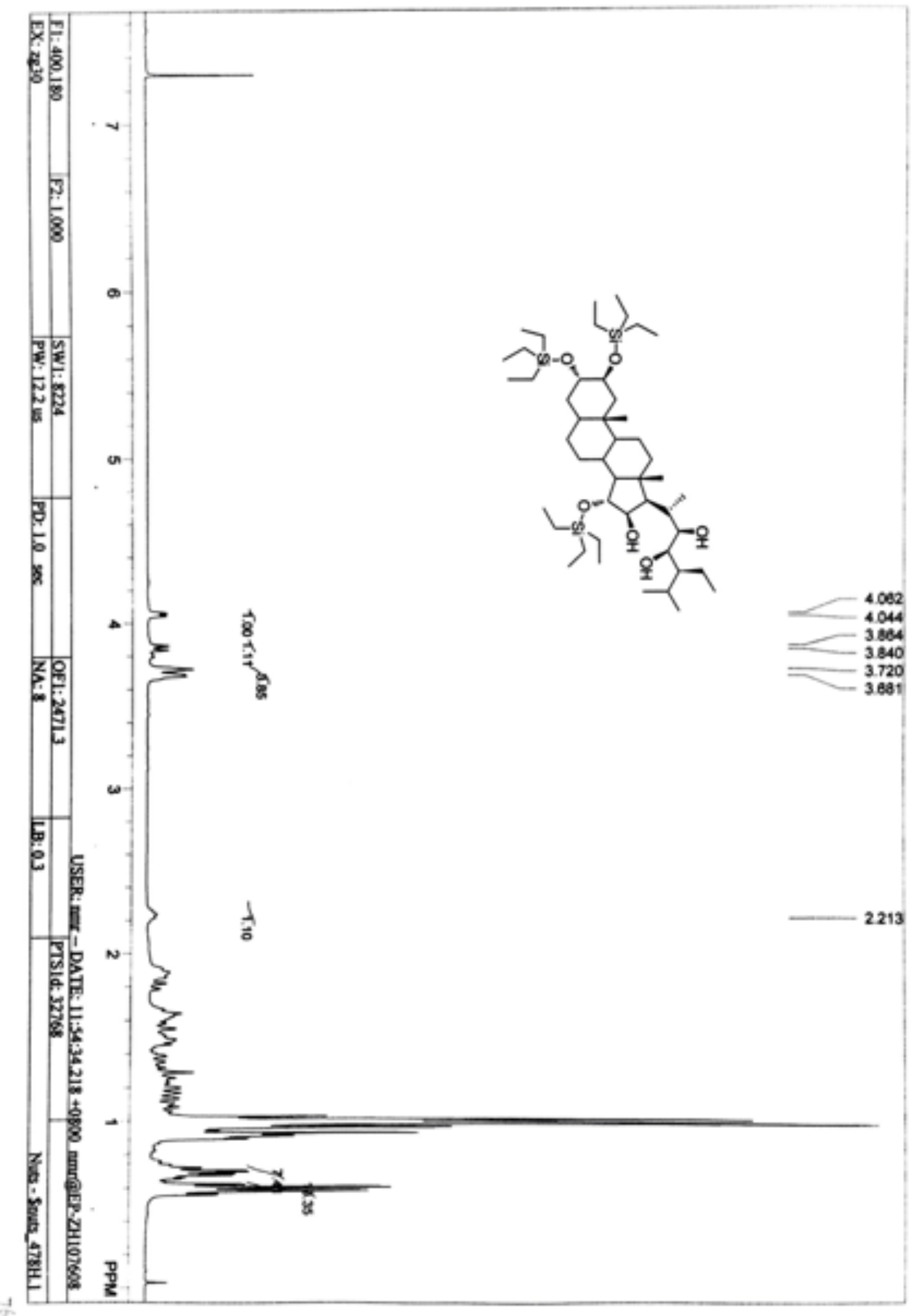


${ }^{13} \mathrm{C}$ NMR DEPT135 spectra of compound $15\left(100 \mathrm{MHz}, \mathrm{CDCl}_{3}\right)$

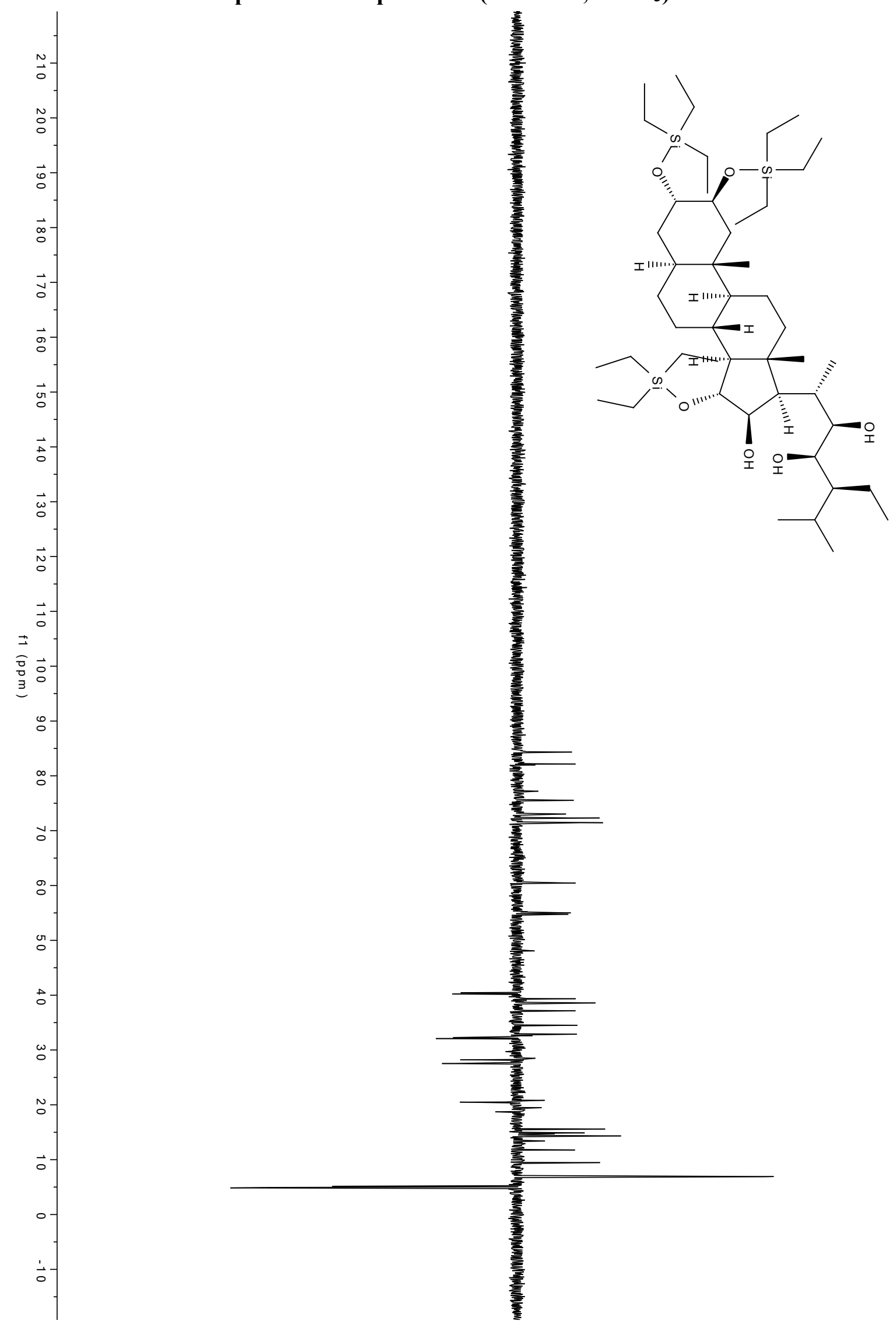


${ }^{1} \mathrm{H}$ NMR spectra of compound $16\left(400 \mathrm{MHz}, \mathrm{CDCl}_{3}\right)$

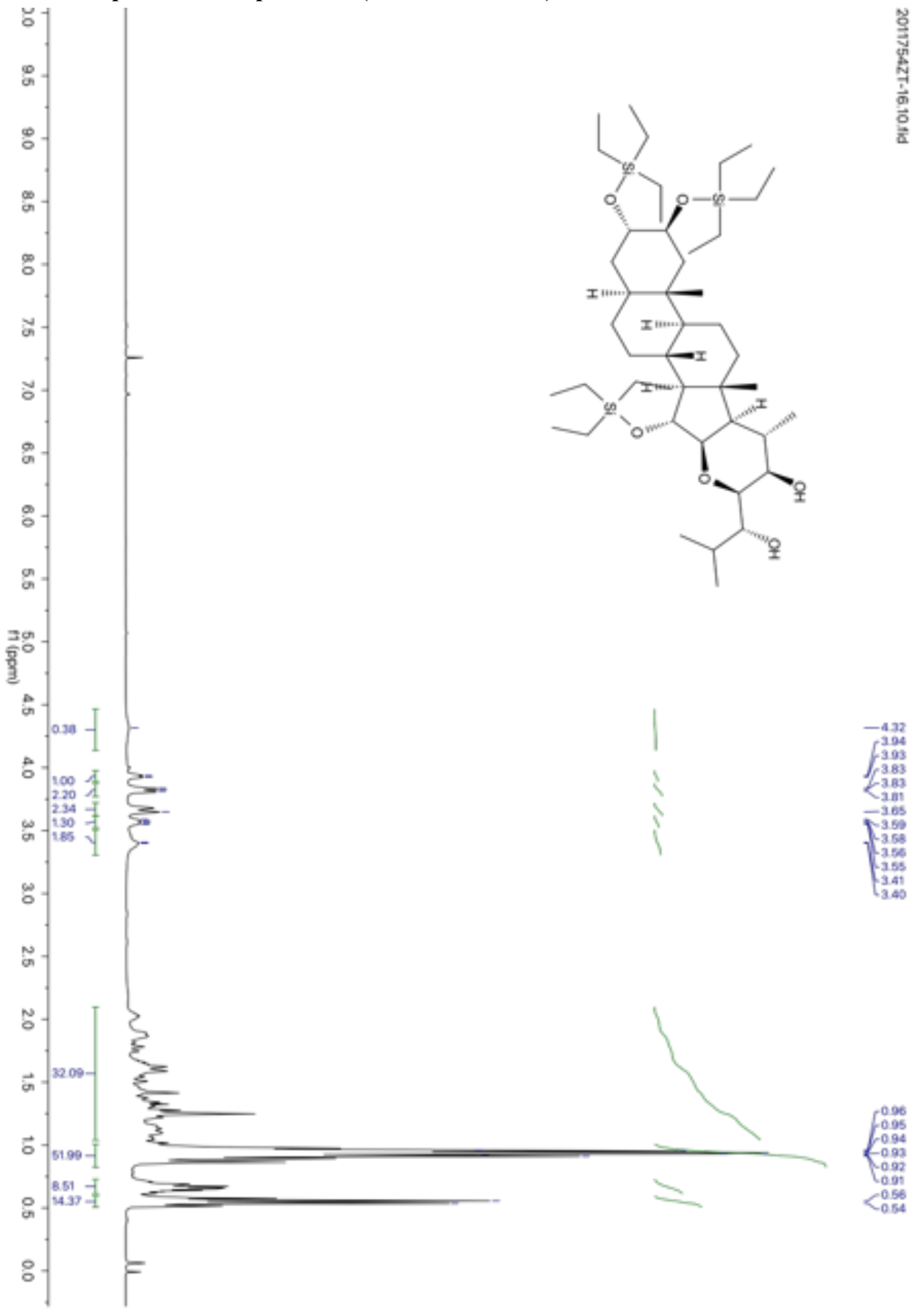


${ }^{13} \mathrm{C}$ NMR spectra of compound $16\left(100 \mathrm{MHz}, \mathrm{CDCl}_{3}\right)$

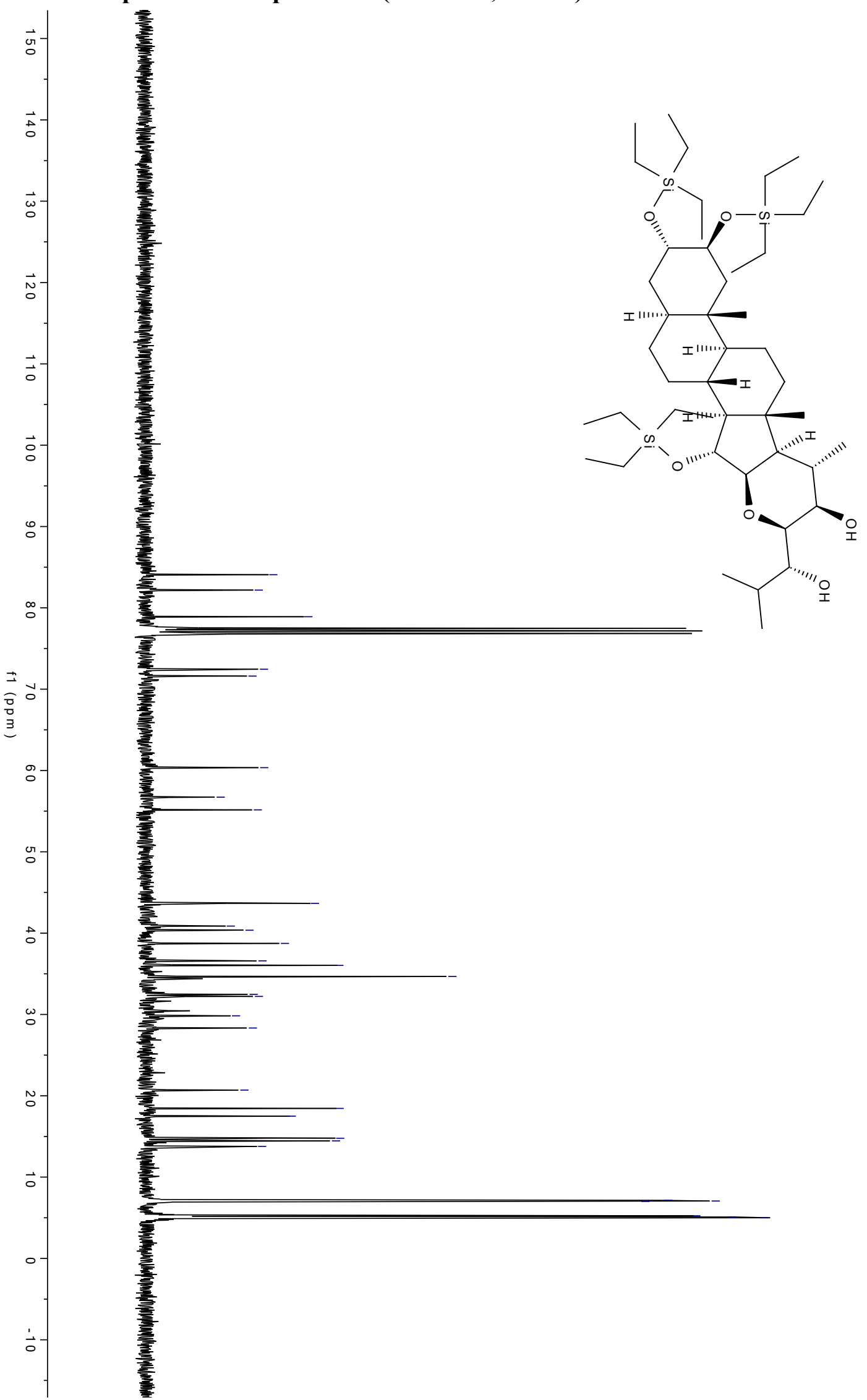

$-84.08$

$\sim 82.18$

$-78.91$

$-72.45$

$\sim 71.61$

$-60.35$

56.72

$-55.16$

43.66

40.87

40.36

38.74

36.59

36.03

34.67

32.48

\32.22

$-29.84$

28.34

20.70

18.45

J 17.49

14.79

$\mathcal{\Upsilon}_{14.46}^{13.78}$

7.15
-7.06

$\left[\begin{array}{l}7.06 \\ 7.01\end{array}\right.$

5.10

5.00 
${ }^{1} \mathrm{H}$ NMR spectra of compound $17\left(400 \mathrm{MHz} \mathrm{CDCl}_{3}\right)$
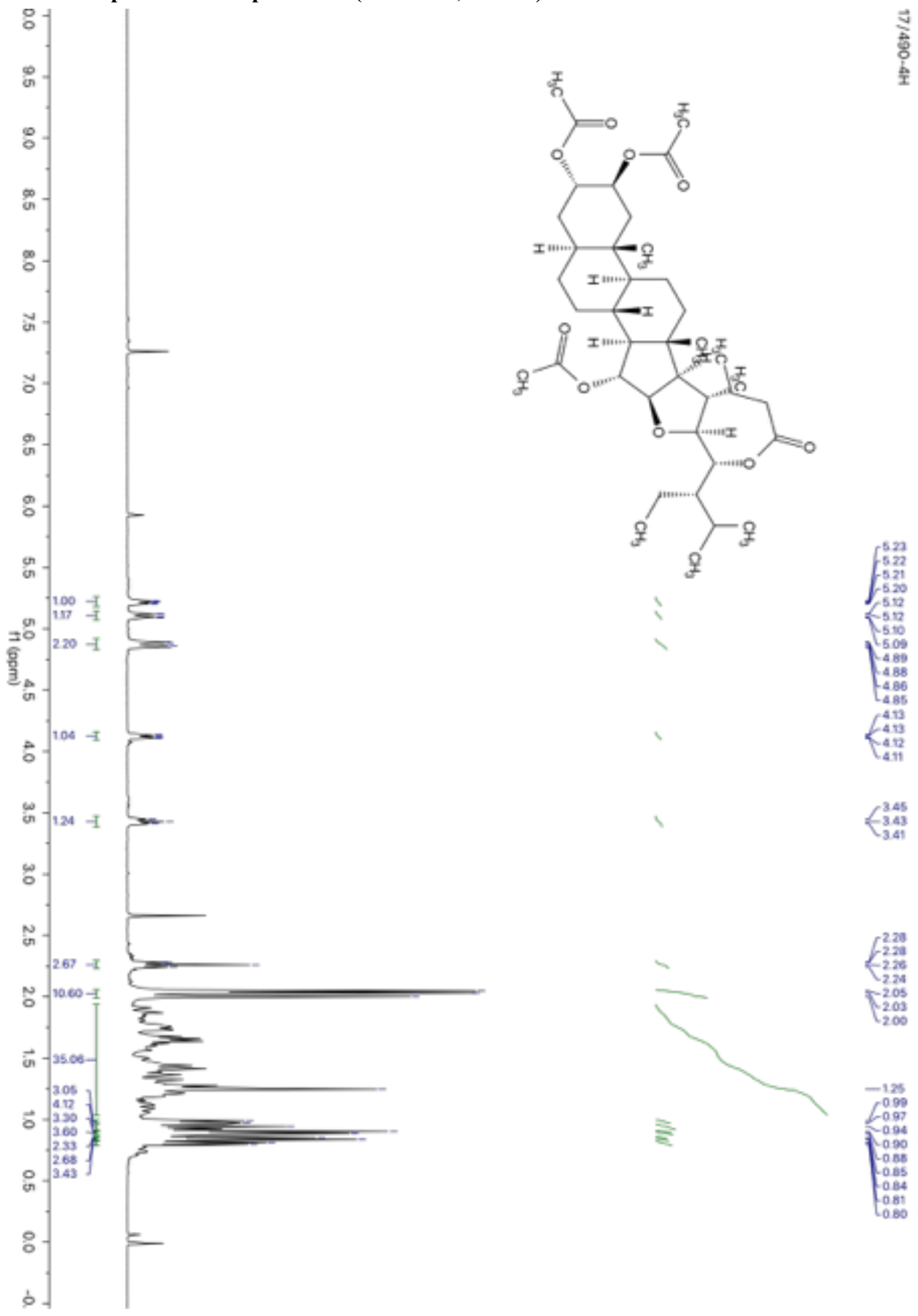
${ }^{13} \mathrm{C}$ NMR spectra of compound $17\left(100 \mathrm{MHz}, \mathrm{CDCl}_{3}\right)$

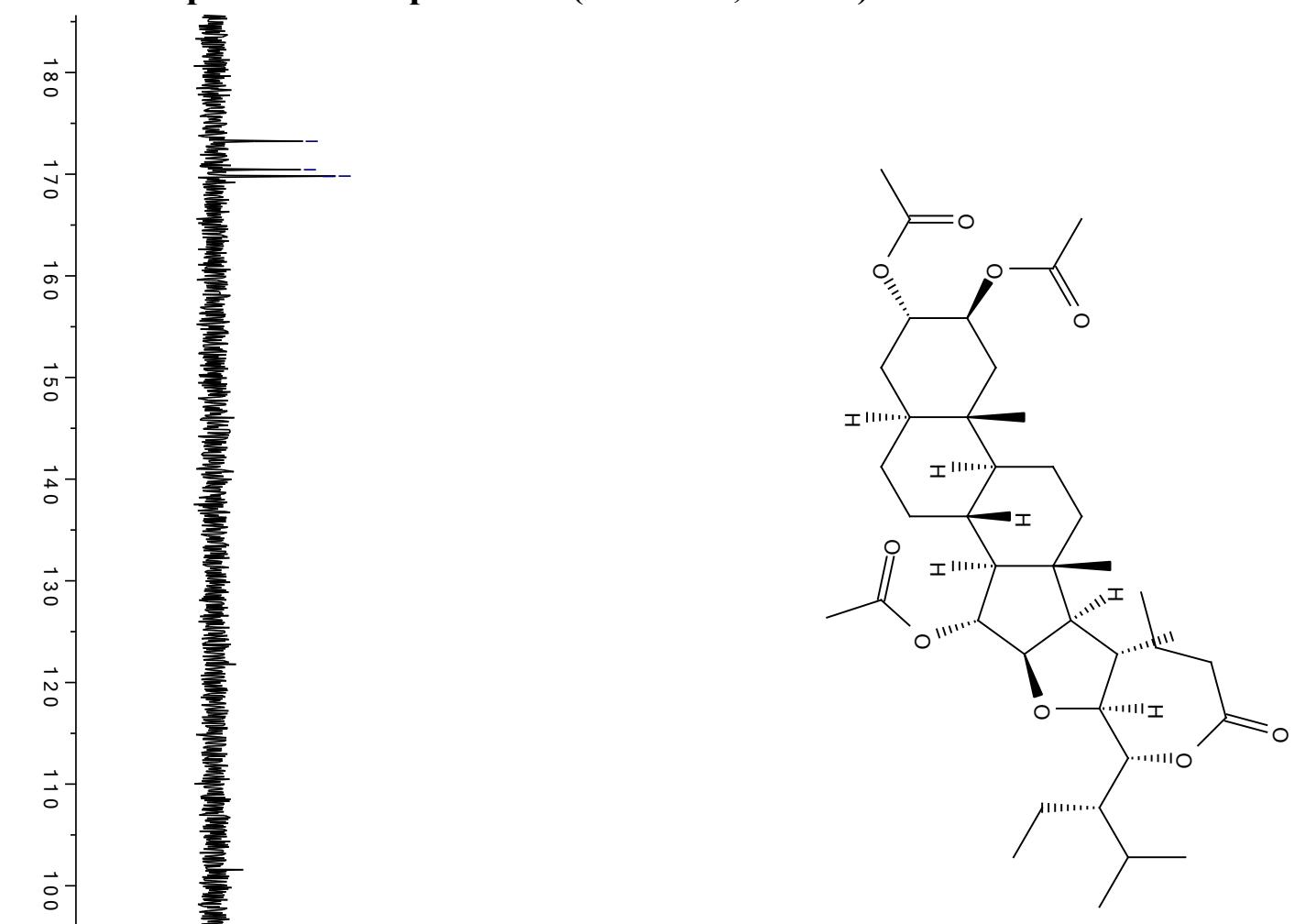

173.23

170.44

T 169.82

169.77

$\overrightarrow{0}$
$\overline{0}$
$\overline{3}$

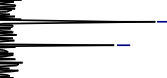

$-90.22$

$-88.11$

$\Upsilon^{80.22}$

59.70

54.79

42.30

$-39.47$

$-37.92$

$-37.81$

$-37.00$

36.63

35.60

35.12

34.62

31.02

29.82

] 29.32

27.78

27.30

21.62

21.42

21.36

21.22

20.38

18.49

17.16

15.80

13.94

13.90

11.58

$\left[\begin{array}{l}11.58 \\ 10.17\end{array}\right.$ 
${ }^{13} \mathrm{C}$ NMR DEPT135 spectra of compound $17\left(100 \mathrm{MHz}, \mathrm{CDCl}_{3}\right)$
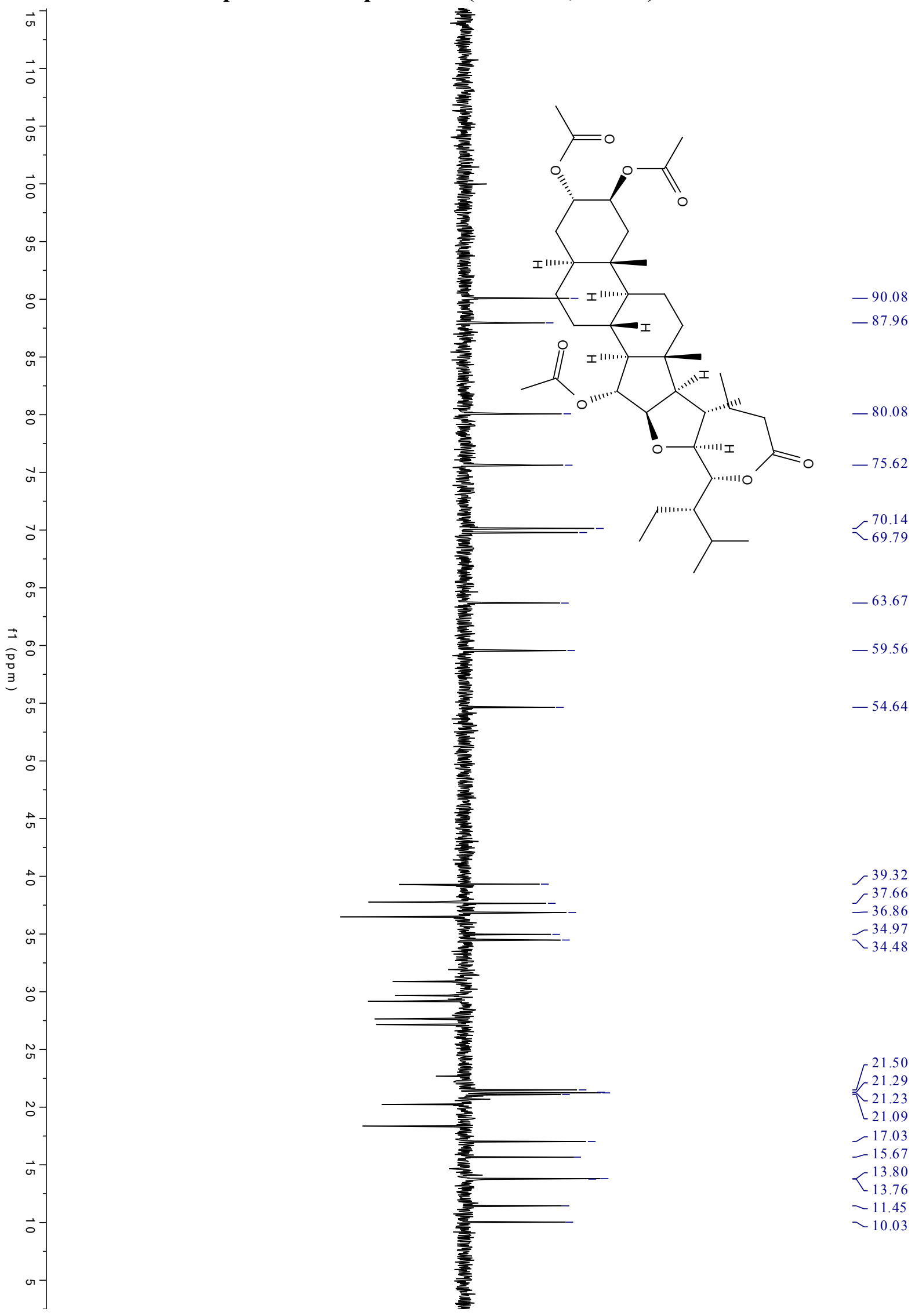

$\sim^{39.32}$

37.66

$-36.86$

$-34.97$

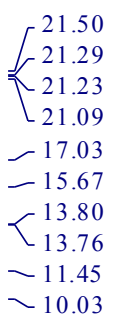




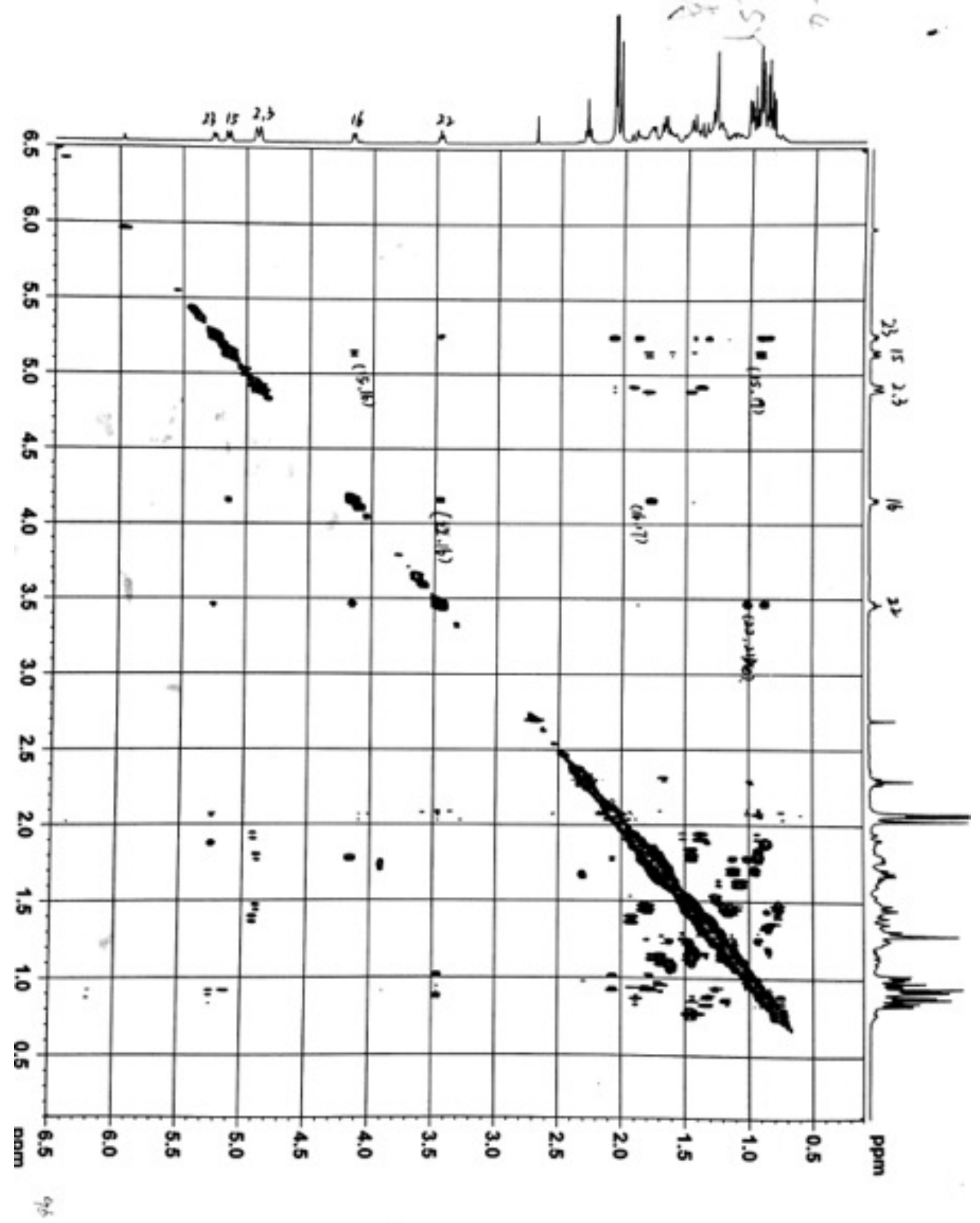




\section{${ }^{1} \mathrm{H}$ NMR spectra of compound $18\left(400 \mathrm{MHz}, \mathrm{CDCl}_{3}\right)$}

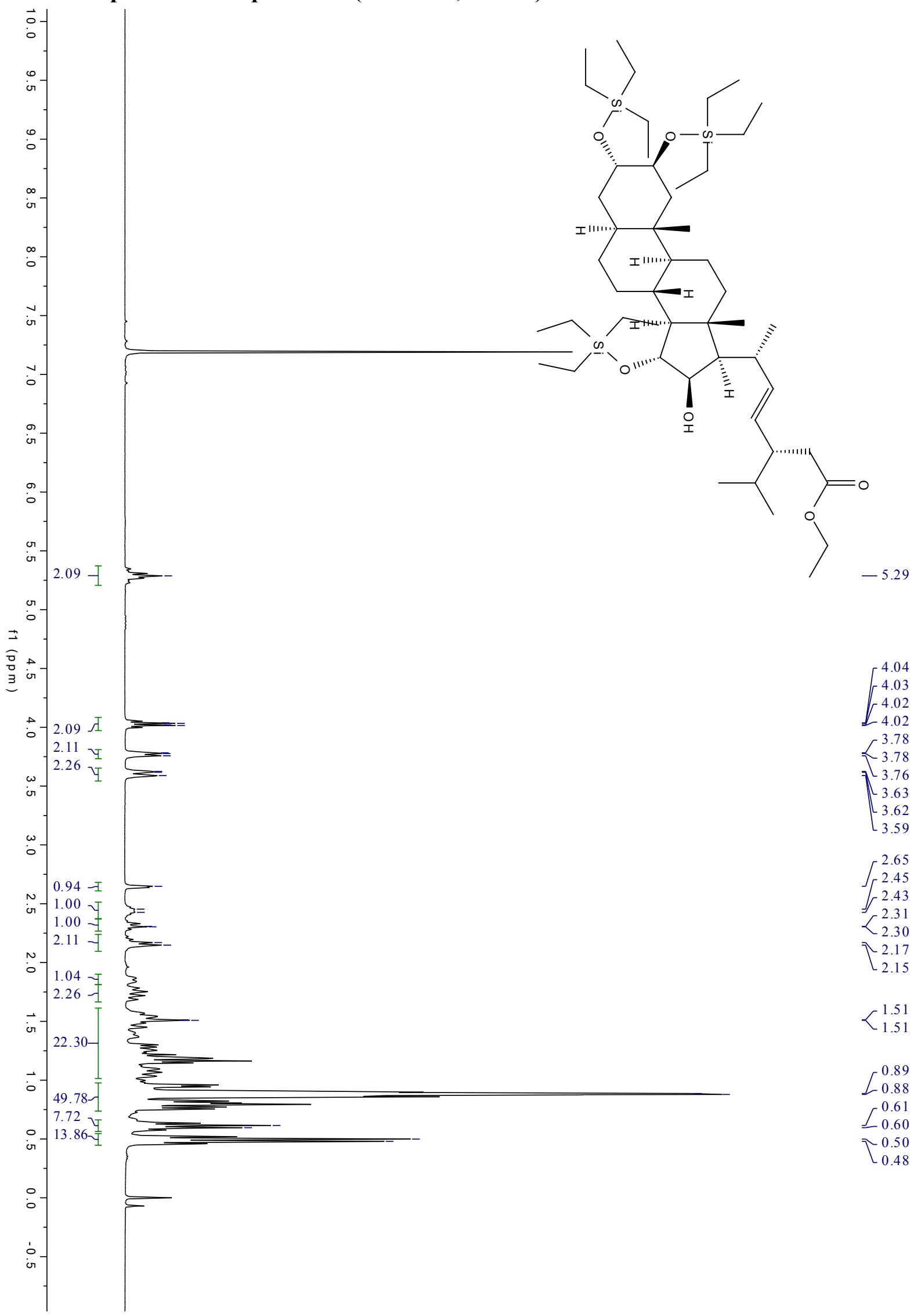


${ }^{13} \mathrm{C}$ NMR spectra of compound $18\left(100 \mathrm{MHz}, \mathrm{CDCl}_{3}\right)$

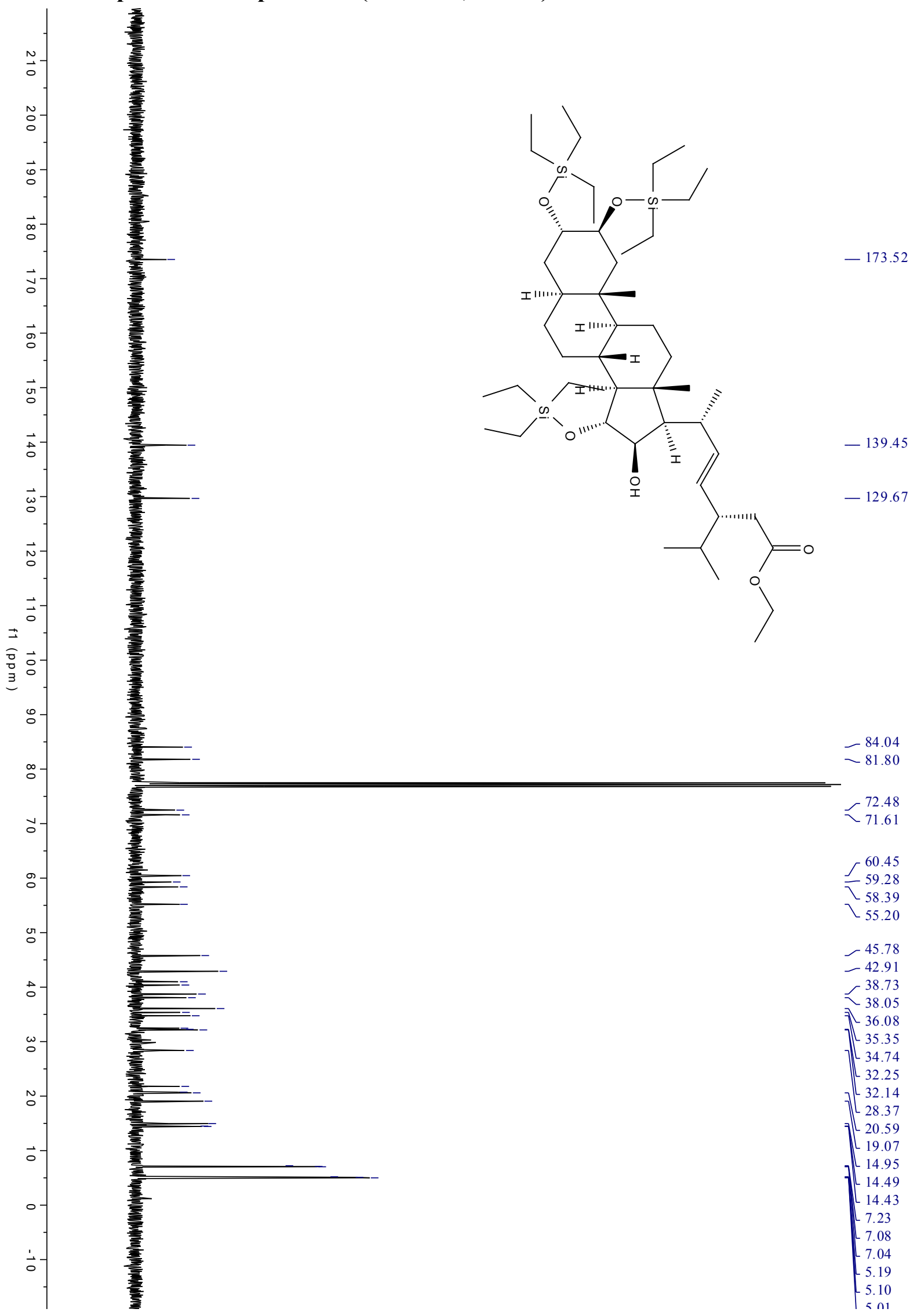




\section{${ }^{1} \mathrm{H}$ NMR spectra of compound $19\left(400 \mathrm{MHz}, \mathrm{CDCl}_{3}\right)$}

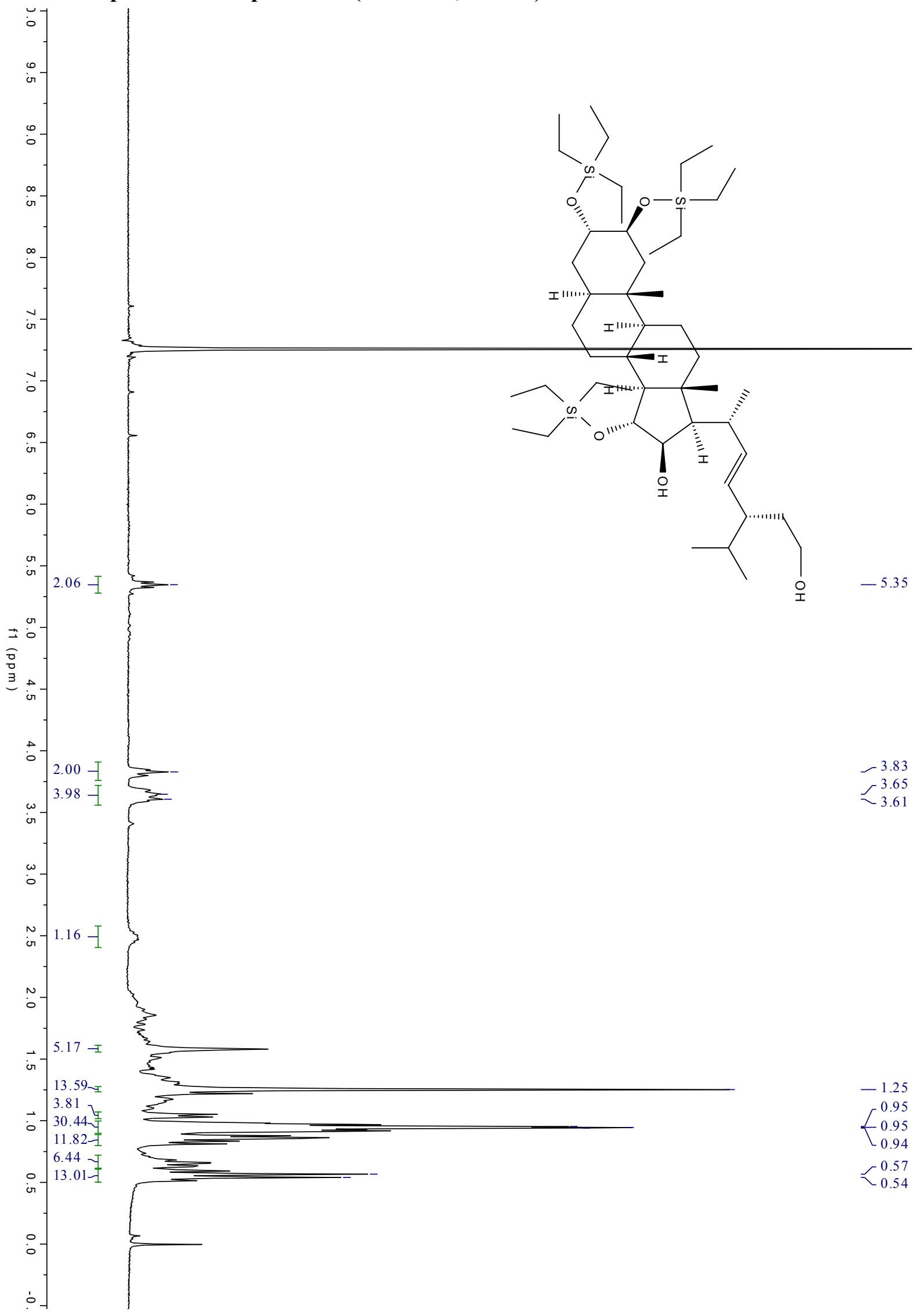


${ }^{13} \mathrm{C}$ NMR spectra of compound $19\left(100 \mathrm{MHz}, \mathrm{CDCl}_{3}\right)$
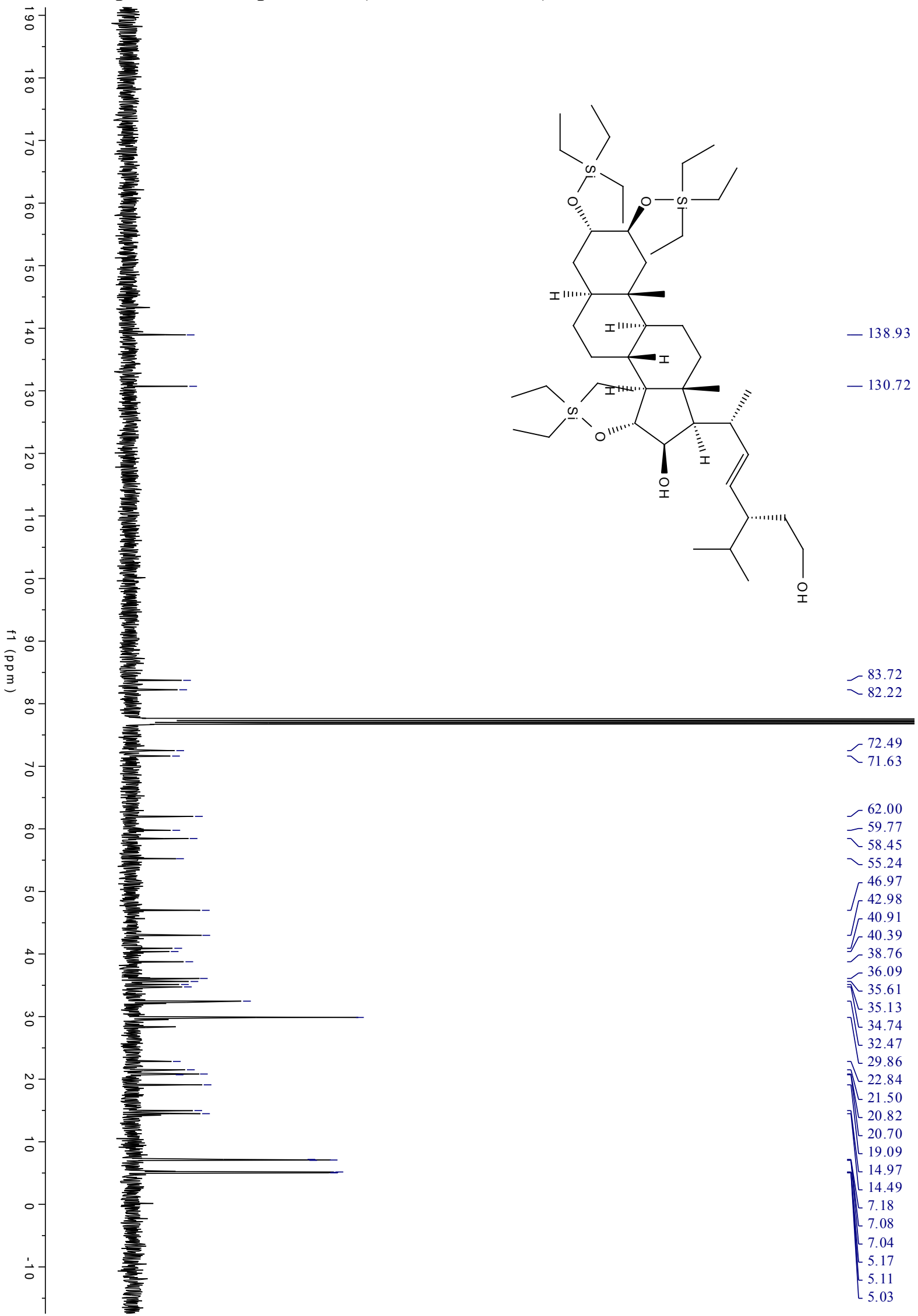

$-83.72$

$\checkmark 82.22$

72.49
71.63

62.00

$-59.77$

入 58.45

$\neg 55.24$

46.97

42.98

40.91

40.39

38.76

36.09

35.61

35.13

34.74

32.47

29.86

ᄂ22.84

21.50

20.82

20.70

19.09

14.97

[4.49

$\left[\begin{array}{l}7.18 \\ 7.08\end{array}\right.$

7.08

7.04

7.17
5.11

[5.03 
${ }^{1} \mathrm{H}$ NMR spectra of compound $23\left(400 \mathrm{MHz}, \mathrm{CDCl}_{3}\right)$

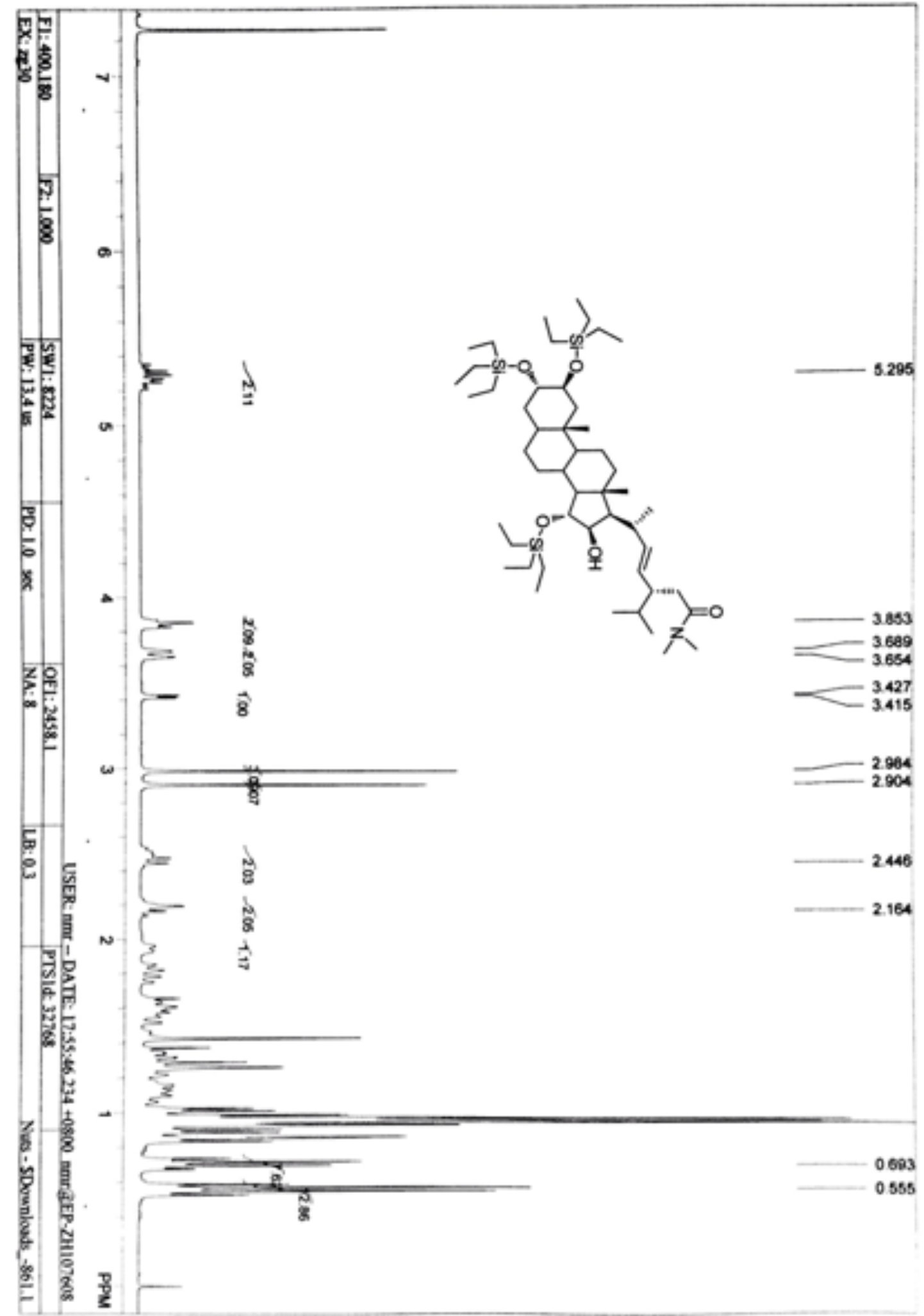


${ }^{13} \mathrm{C}$ NMR spectra of compound $23\left(100 \mathrm{MHz}, \mathrm{CDCl}_{3}\right)$

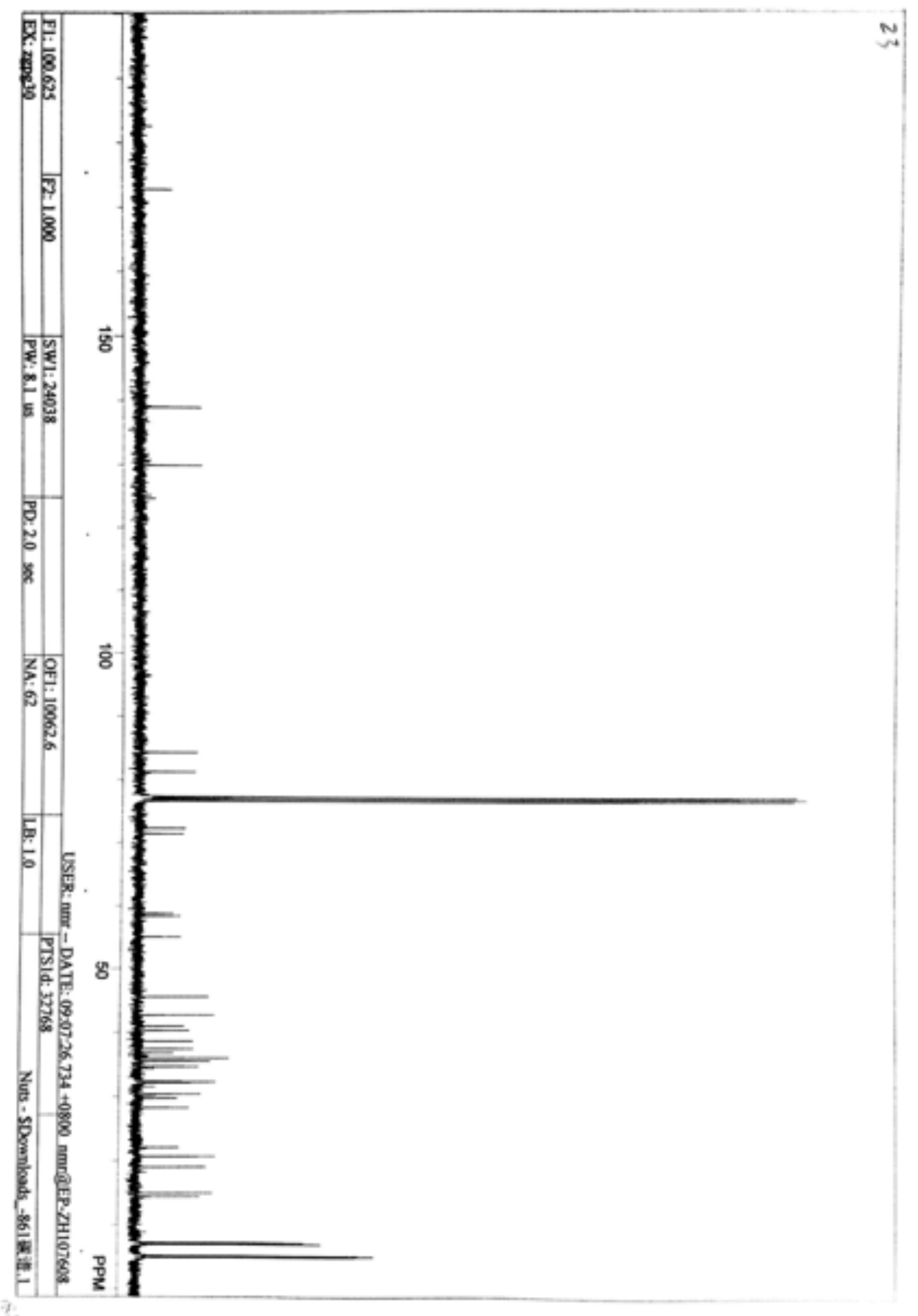


${ }^{1} \mathrm{H}$ NMR spectra of compound $24\left(400 \mathrm{MHz}, \mathrm{CDCl}_{3}\right)$

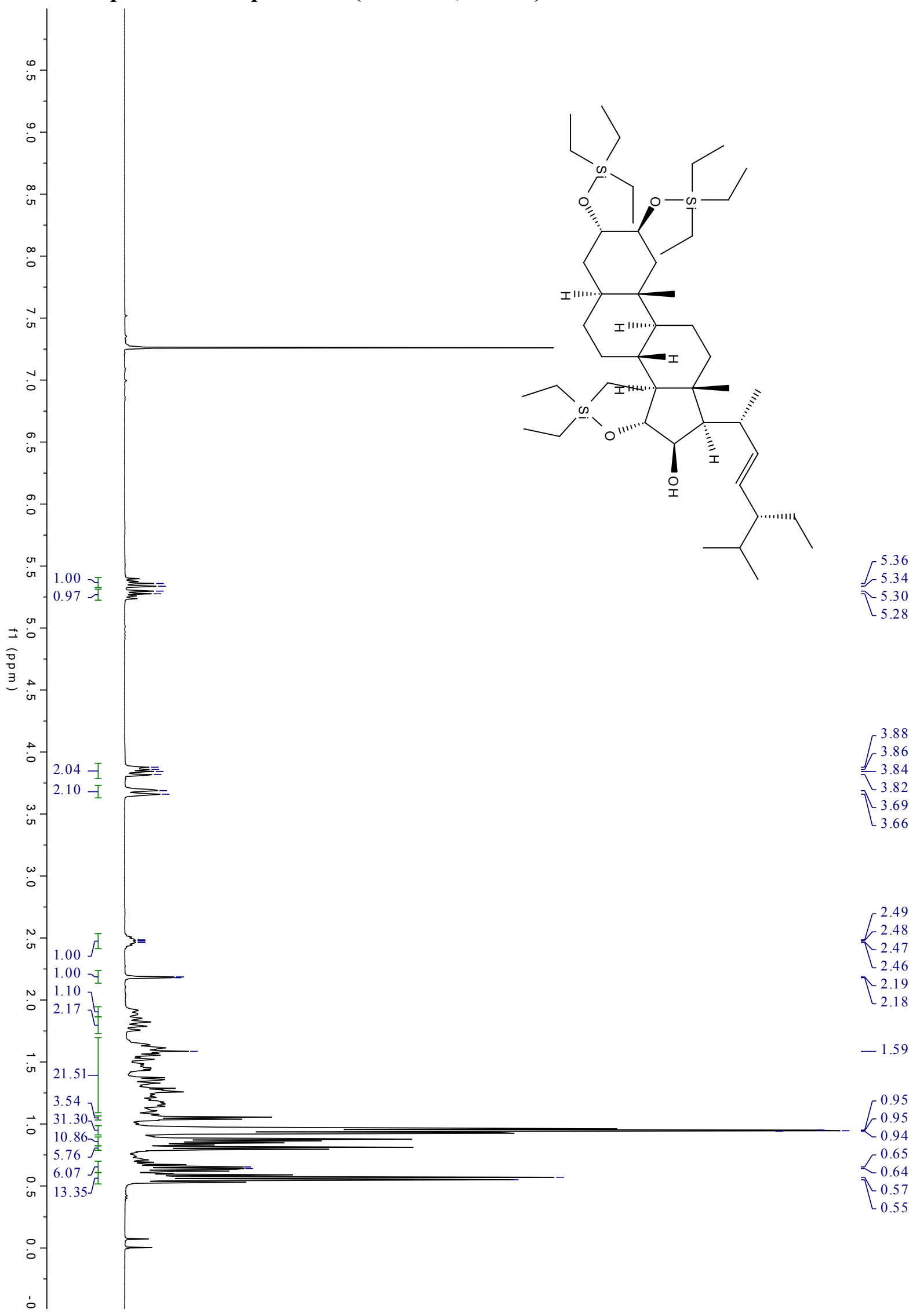


${ }^{13} \mathrm{C}$ NMR spectra of compound $24\left(100 \mathrm{MHz}, \mathrm{CDCl}_{3}\right)$

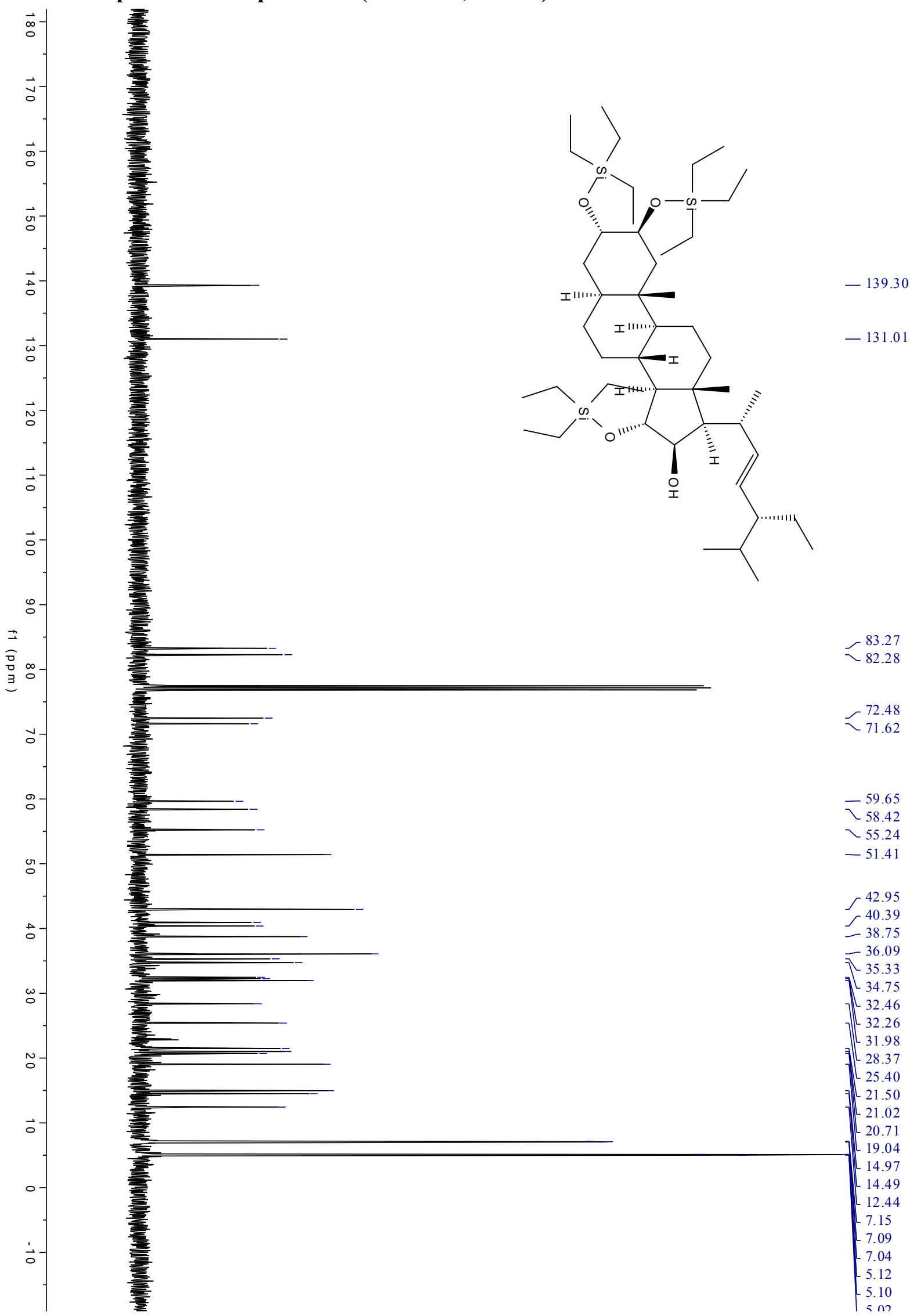


${ }^{1} \mathrm{H}$ NMR spectra of compound S5 (400 $\left.\mathrm{MHz}, \mathrm{CDCl}_{3}\right)$
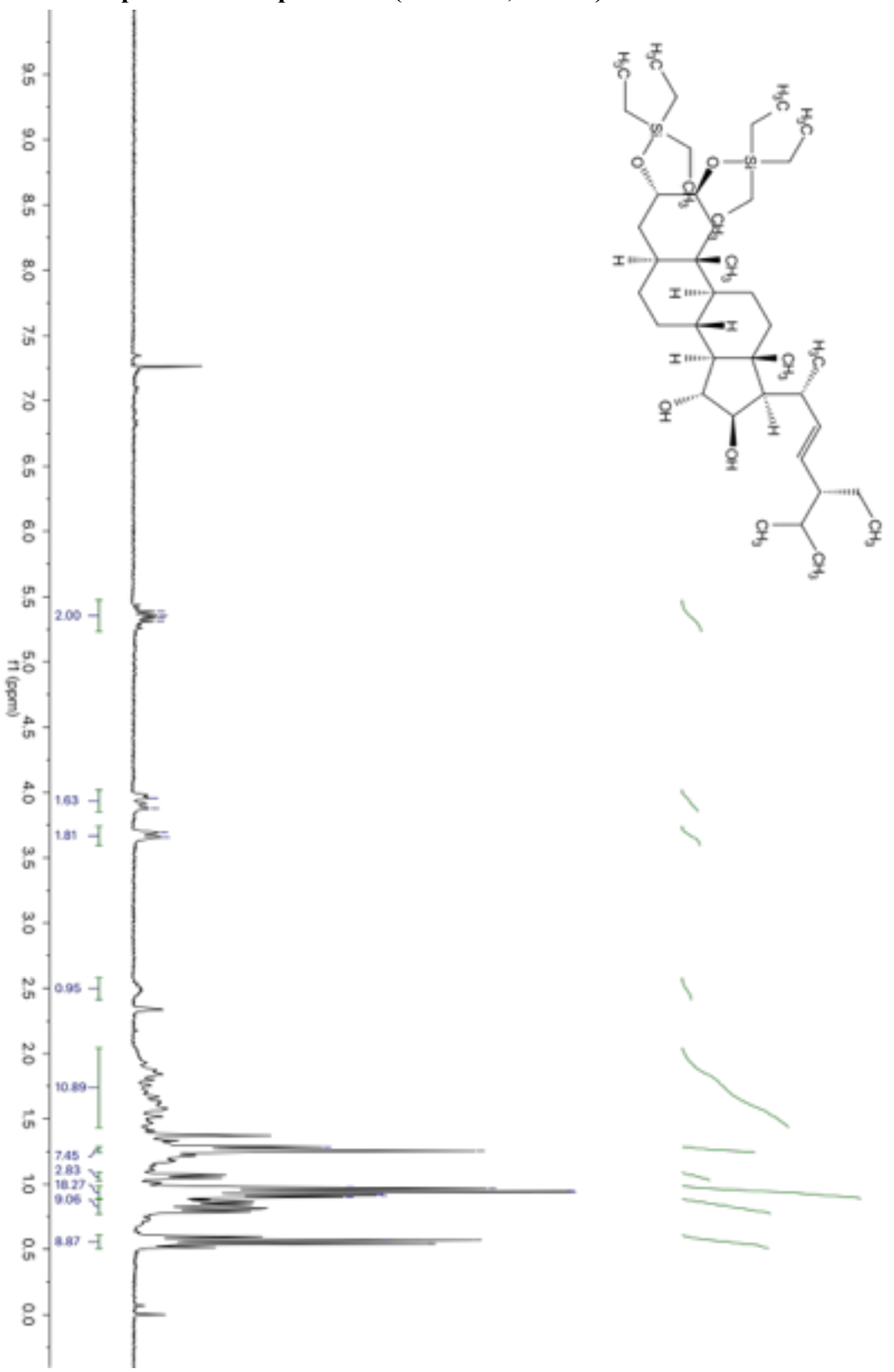
${ }^{1} \mathrm{H}$ NMR spectra of compound $25\left(400 \mathrm{MHz}, \mathrm{CDCl}_{3}\right)$

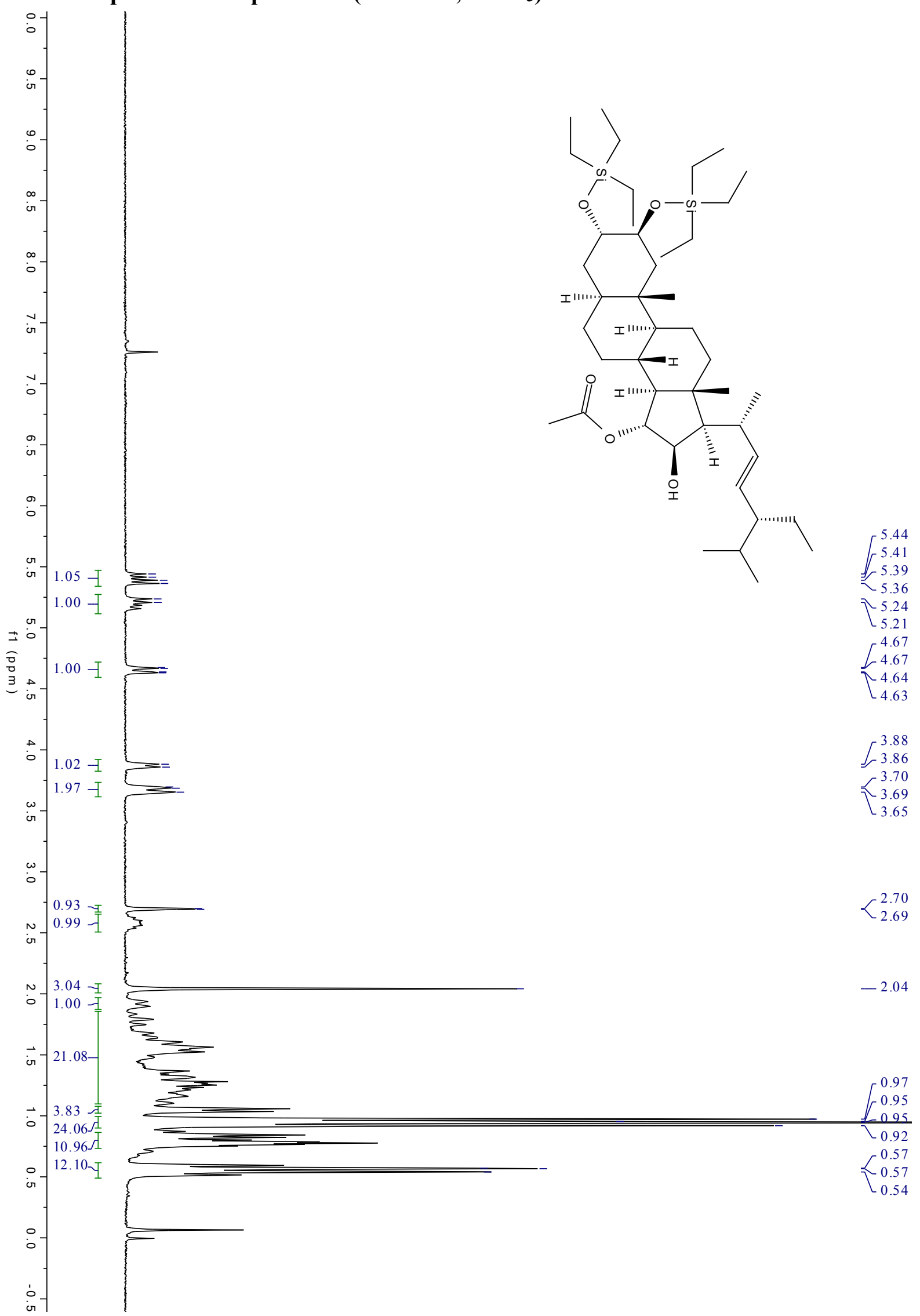


${ }^{1} \mathrm{H}$ NMR spectra of compound $\mathrm{S6}\left(400 \mathrm{MHz}, \mathrm{CDCl}_{3}\right)$

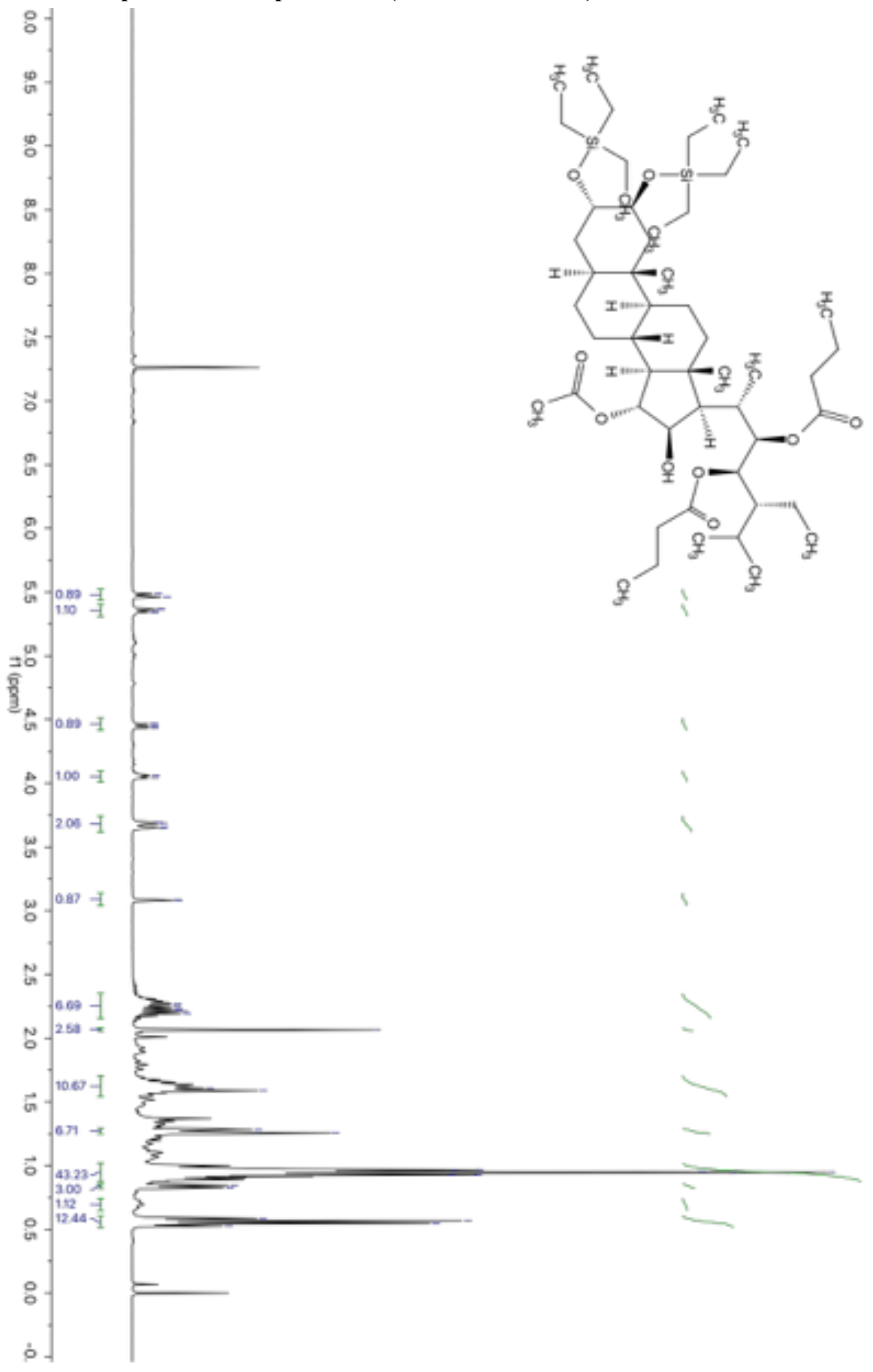


${ }^{13} \mathrm{C}$ NMR spectra of compound $\mathrm{S6}\left(100 \mathrm{MHz}, \mathrm{CDCl}_{3}\right)$

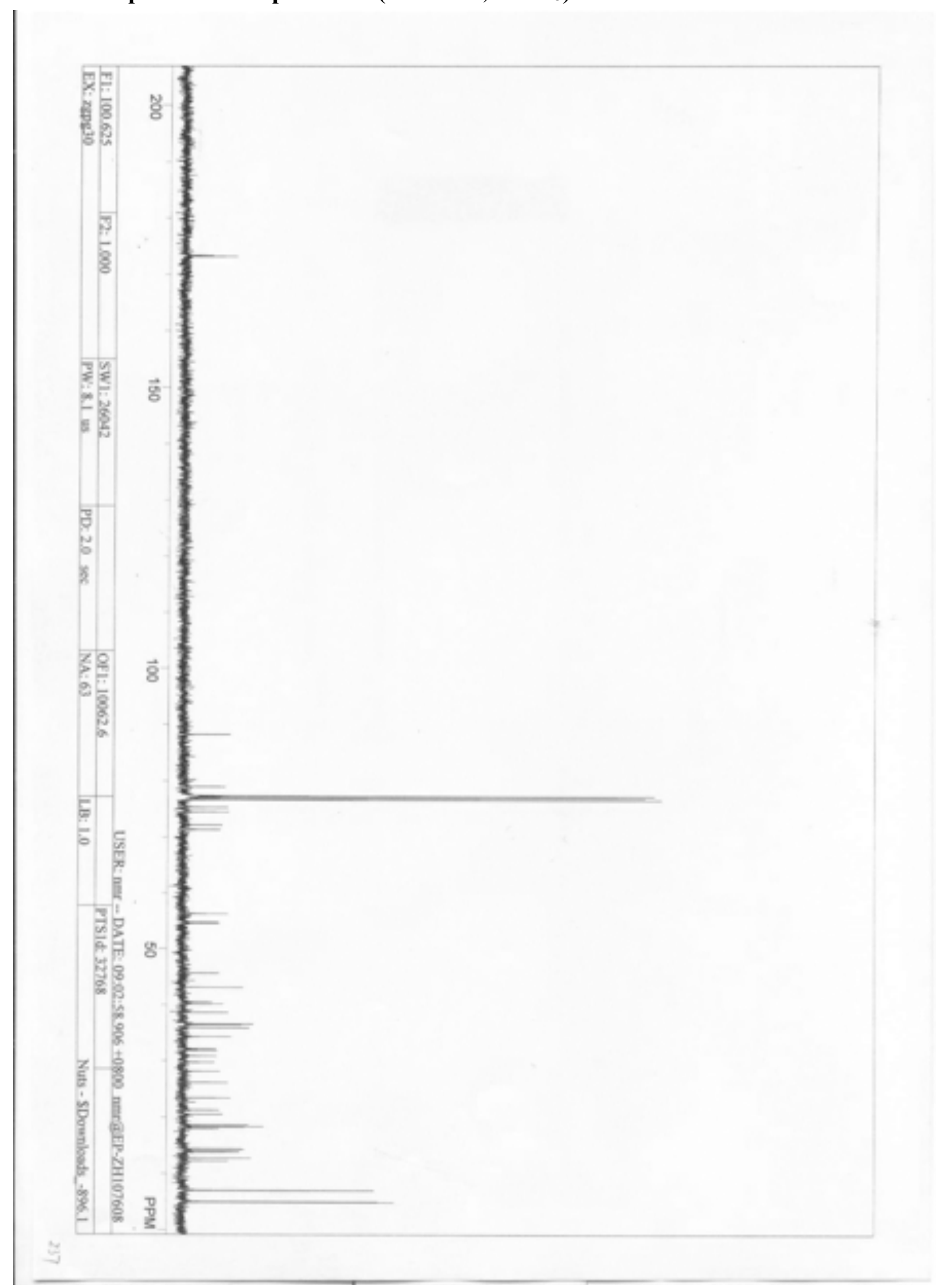


${ }^{1} \mathrm{H}$ NMR spectra of compound $27\left(400 \mathrm{MHz}, \mathrm{CDCl}_{3}\right)$

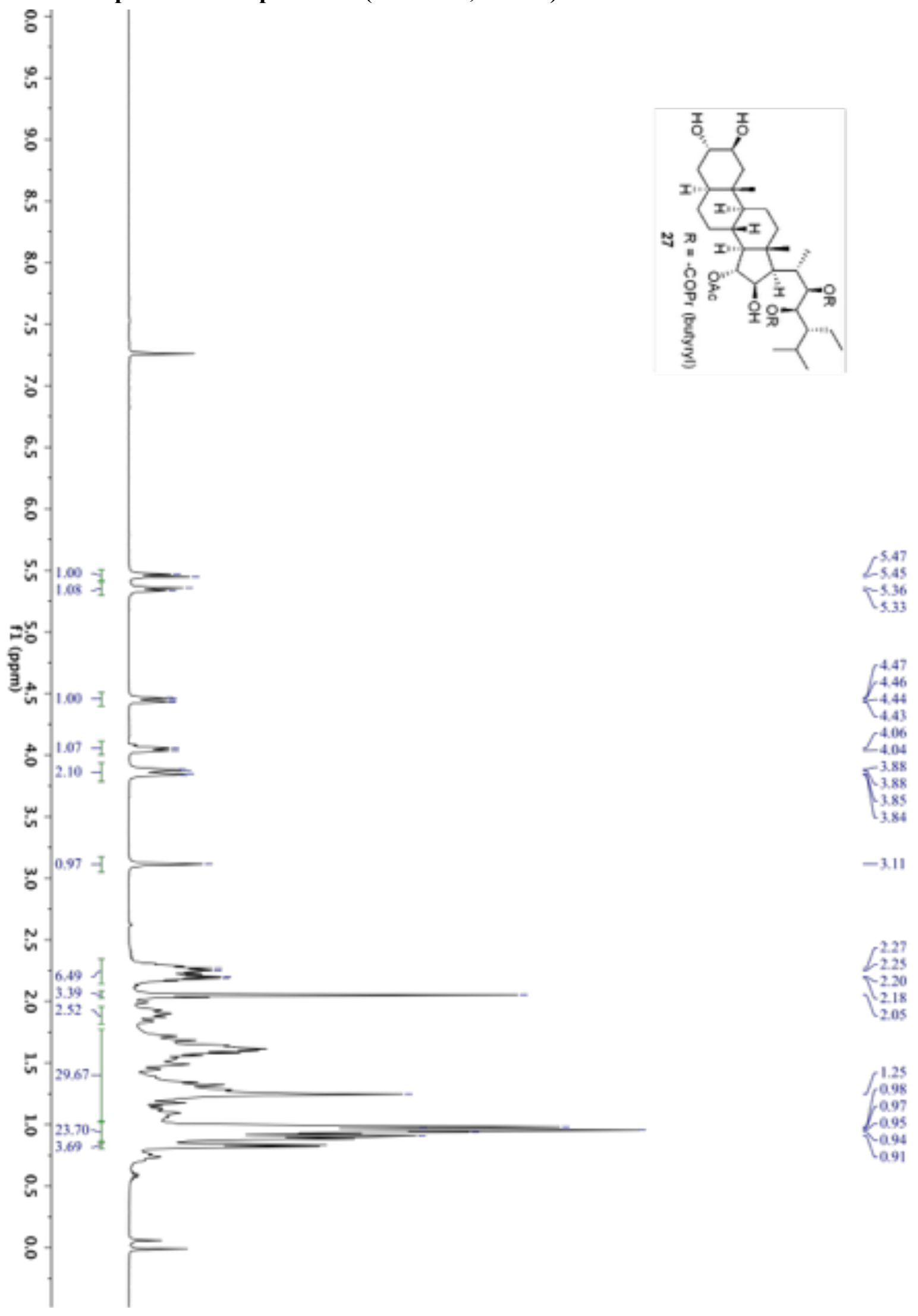


${ }^{13} \mathrm{C}$ NMR spectra of compound $27\left(100 \mathrm{MHz}, \mathrm{CDCl}_{3}\right)$

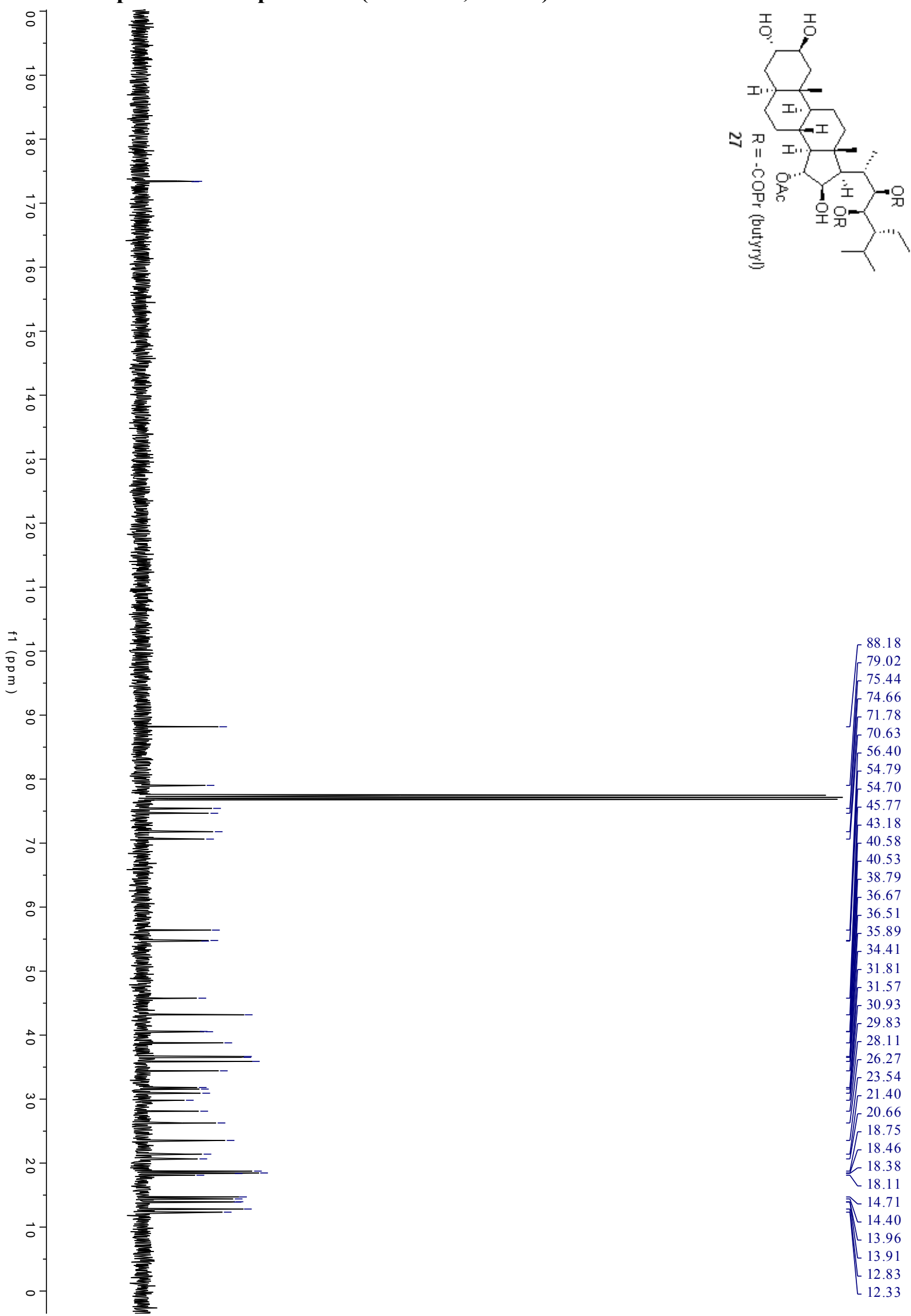


${ }^{1} \mathrm{H}$ NMR spectra of compound $\mathrm{S8}\left(400 \mathrm{MHz}, \mathrm{CDCl}_{3}\right)$

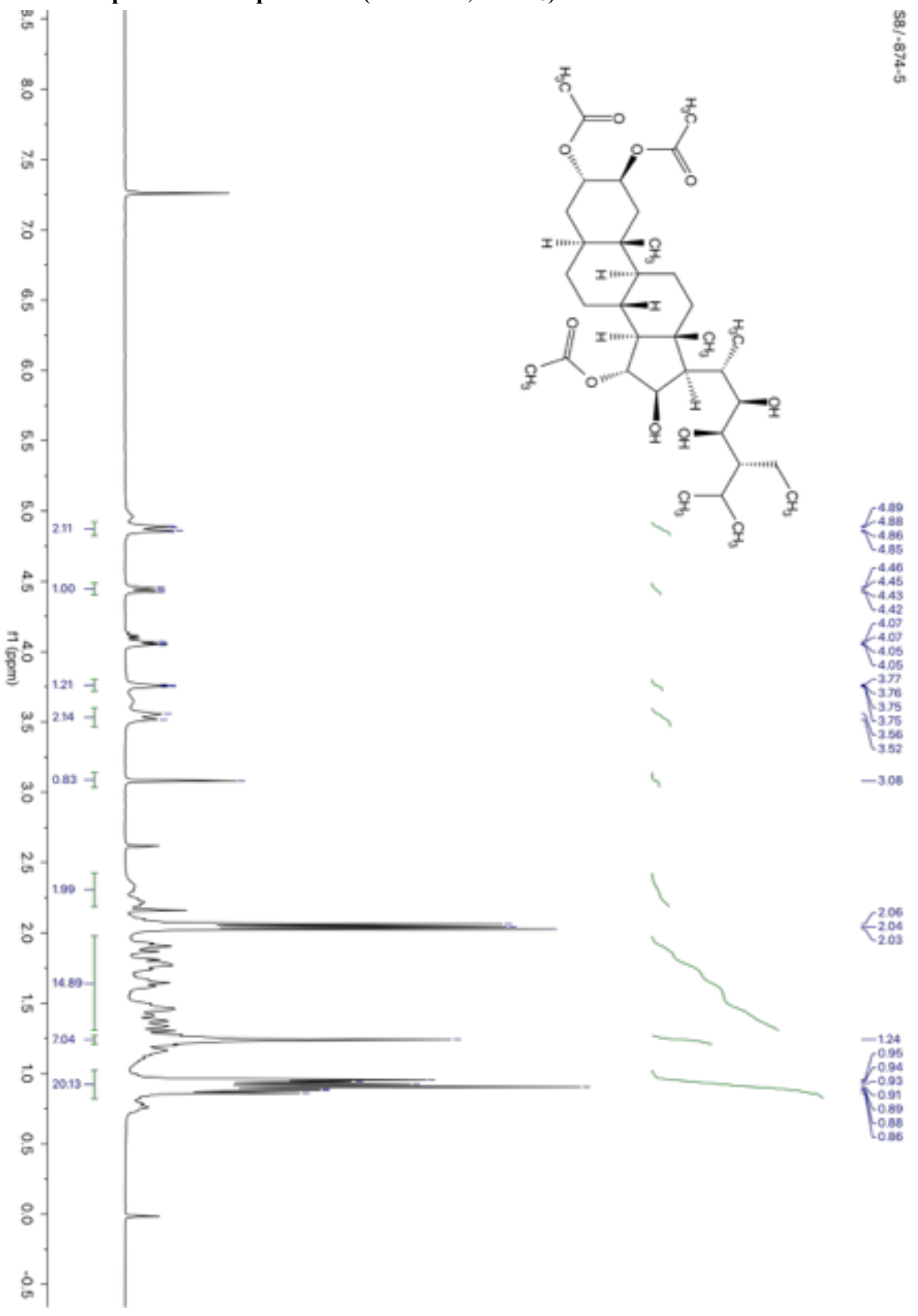


${ }^{13} \mathrm{C}$ NMR spectra of compound S8 (100 $\left.\mathrm{MHz}, \mathrm{CDCl}_{3}\right)$

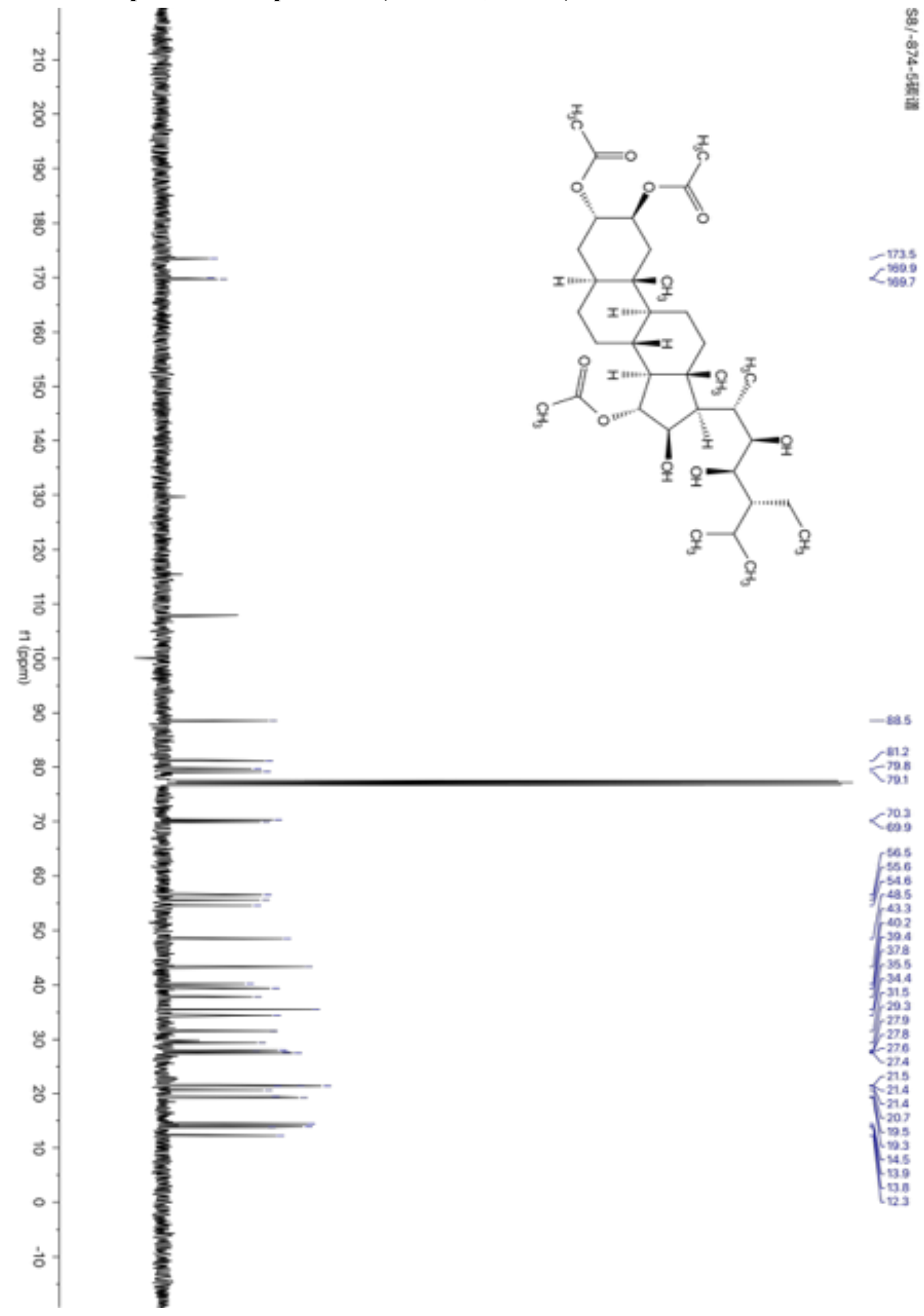


${ }^{1} \mathrm{H}$ NMR spectra of compound S9 (400 $\left.\mathrm{MHz}, \mathrm{CDCl}_{3}\right)$

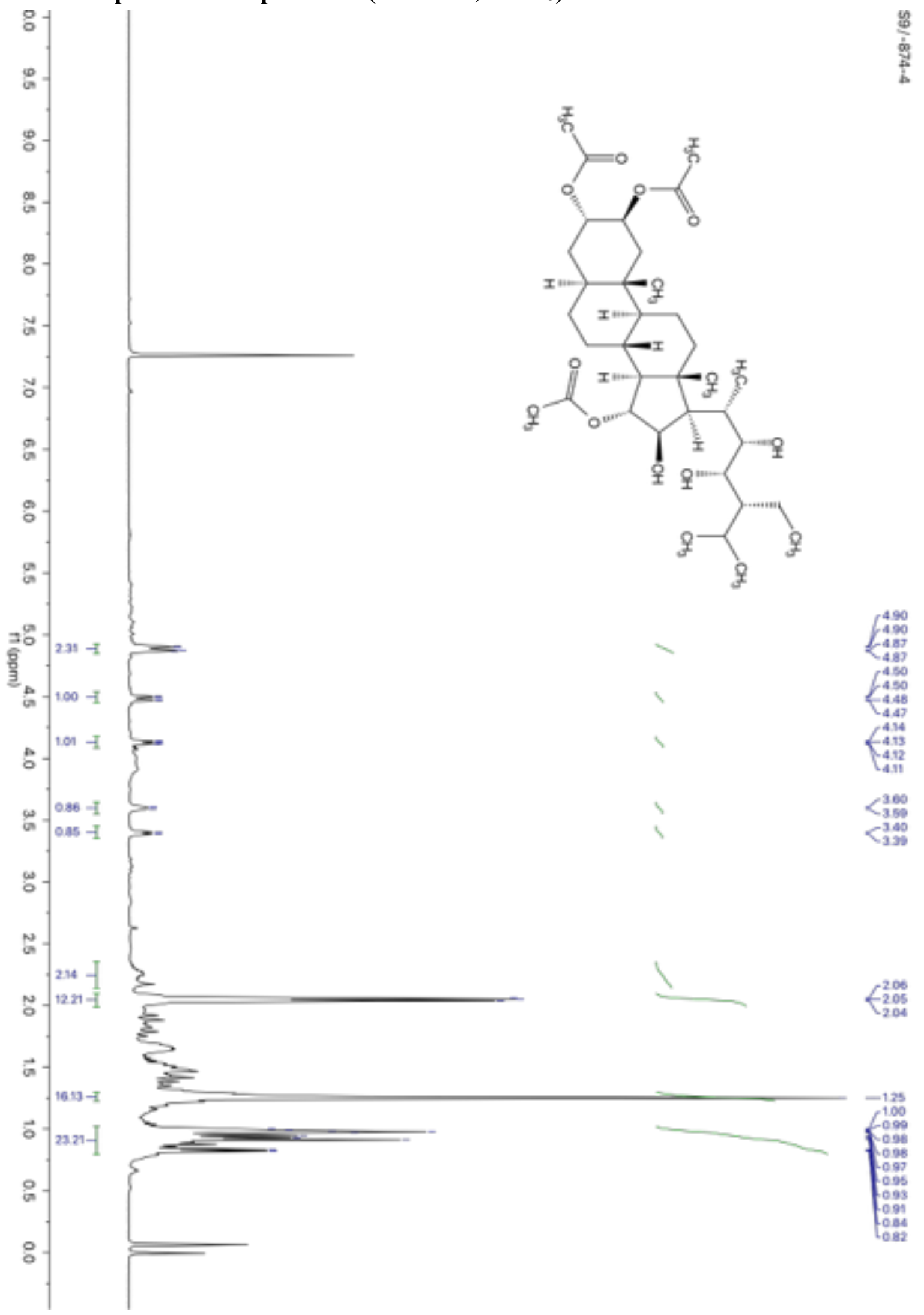


${ }^{13} \mathrm{C}$ NMR spectra of compound $\mathrm{S9}\left(100 \mathrm{MHz} \mathrm{CDCl}_{3}\right)$

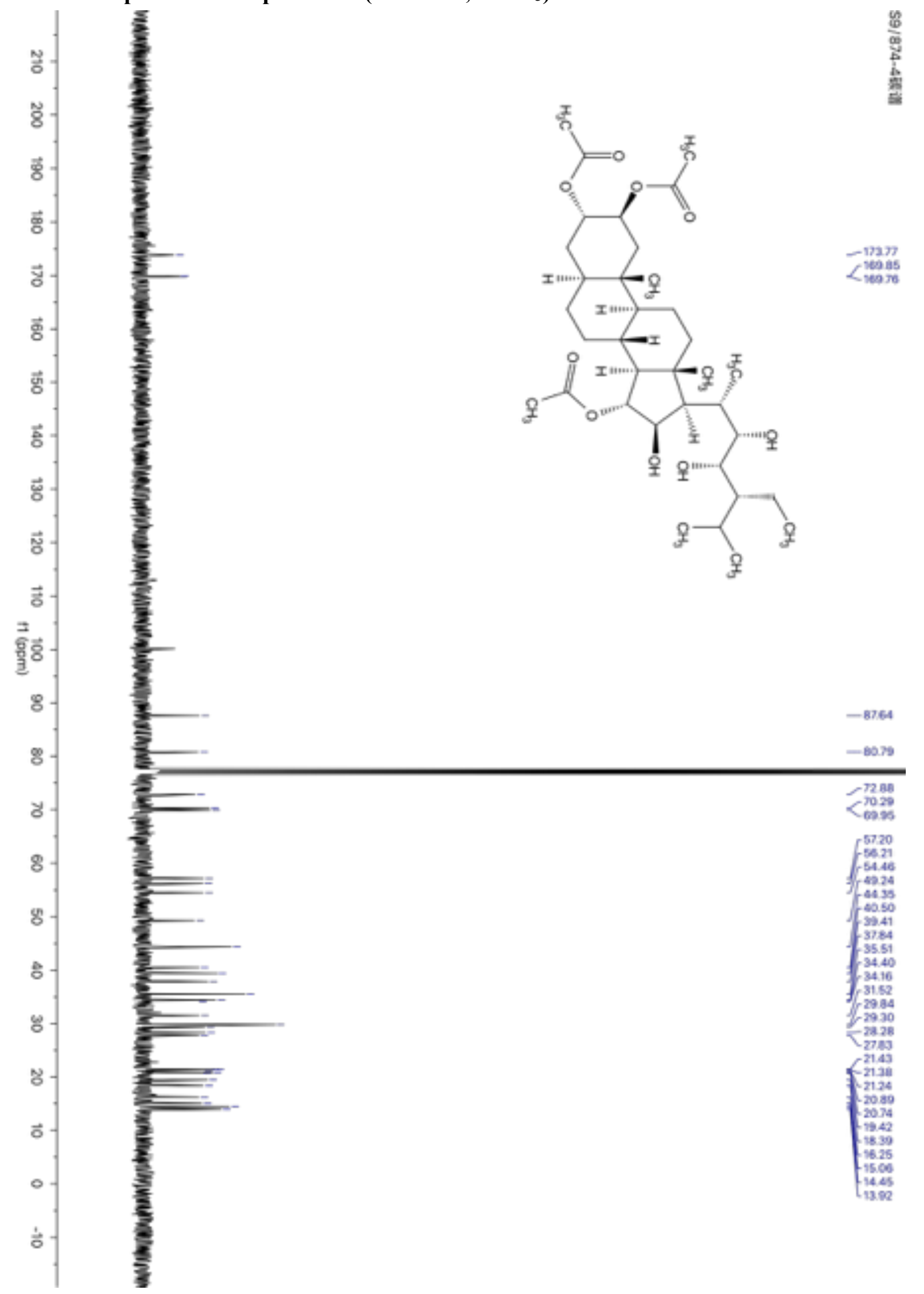


${ }^{1} \mathrm{H}$ NMR spectra of compound $\mathrm{S10}\left(400 \mathrm{MHz}, \mathrm{CDCl}_{3}\right)$

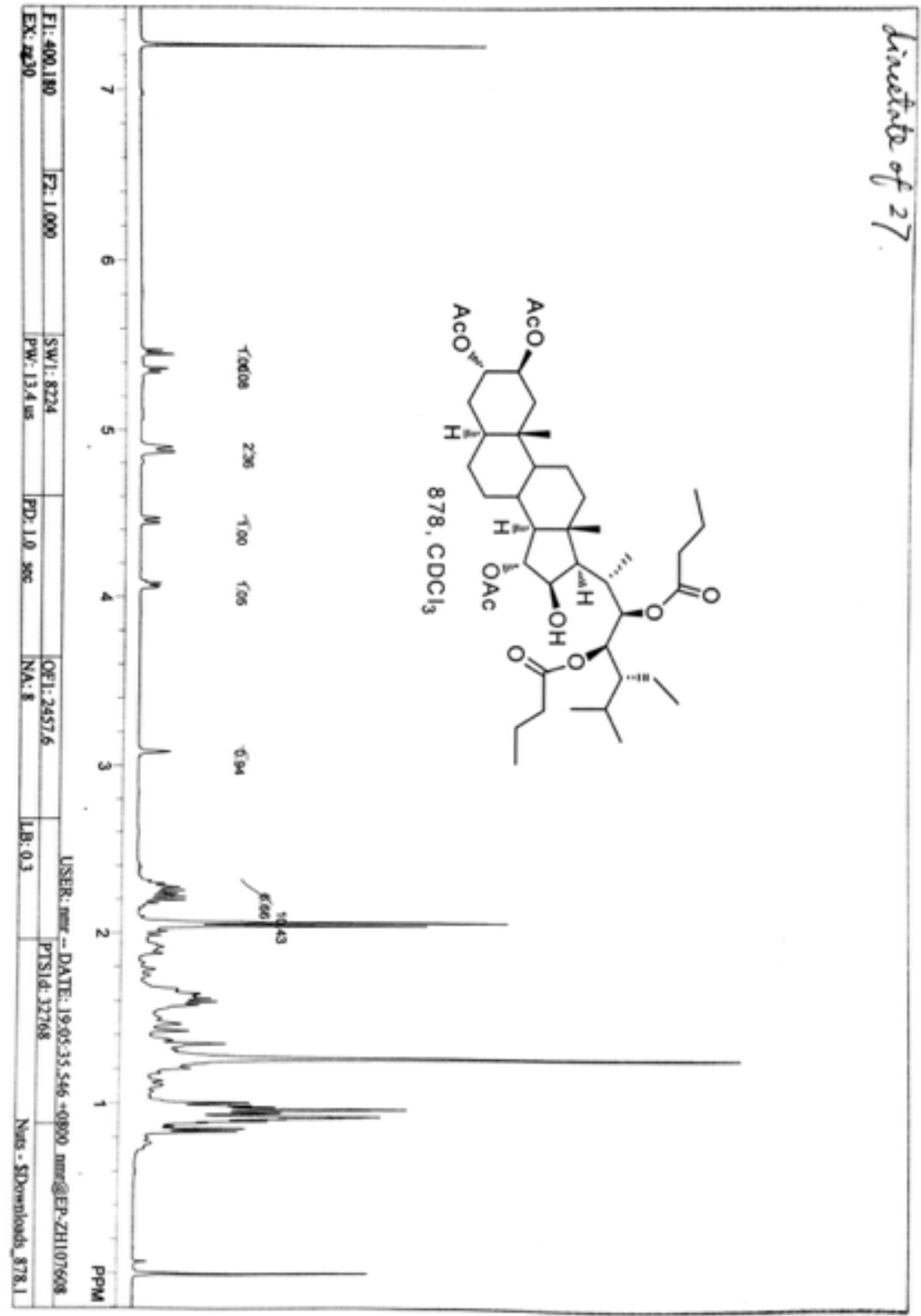


${ }^{1}$ H NMR spectra of compound S10 (400 MHz, DMSO-d6)

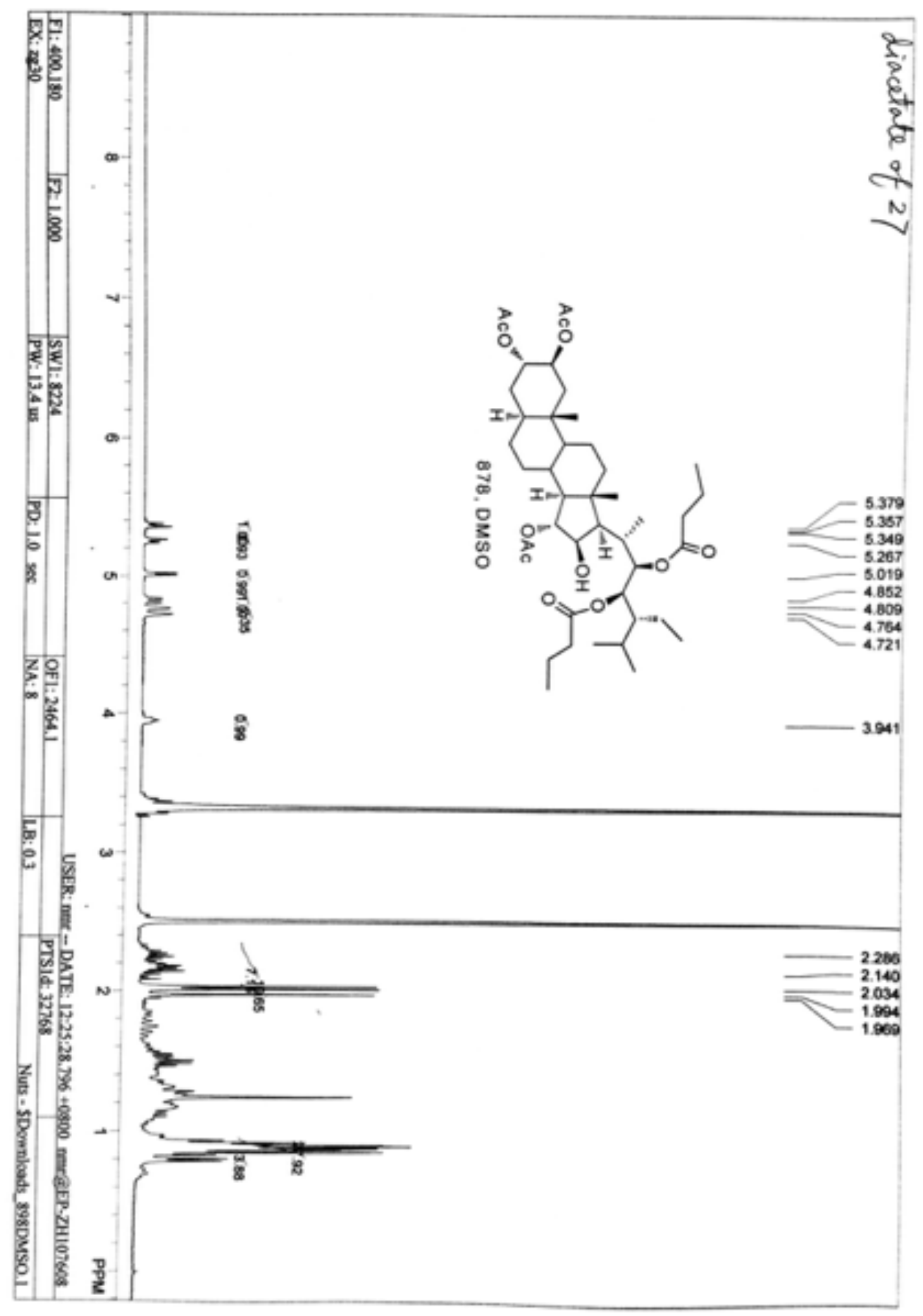


${ }^{1} \mathrm{H}$ NMR spectra of compound S11 (400 $\left.\mathrm{MHz}, \mathrm{CDCl}_{3}\right)$

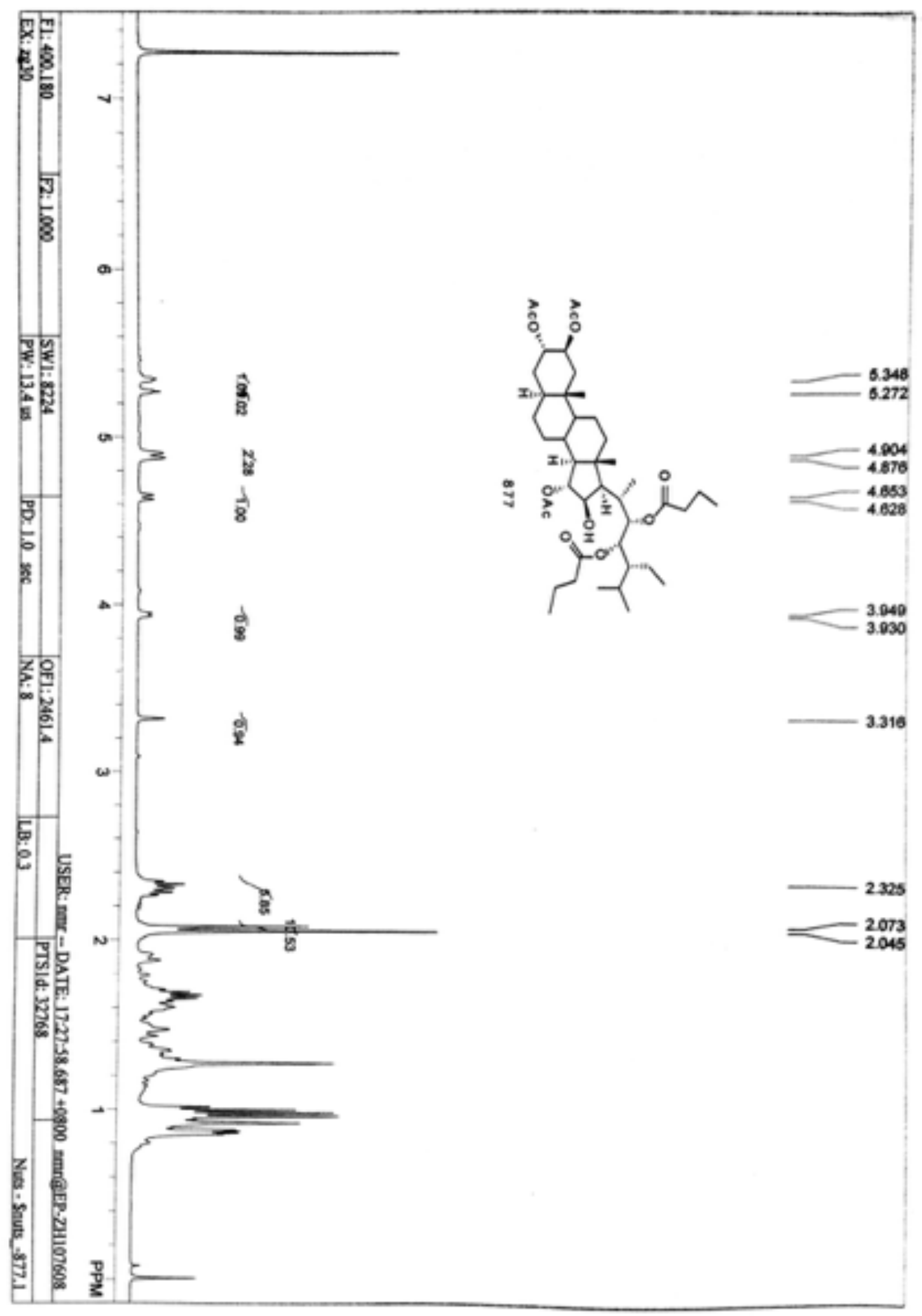


${ }^{1} \mathrm{H}$ NMR spectra of compound $28\left(400 \mathrm{MHz}, \mathrm{CDCl}_{3}\right)$

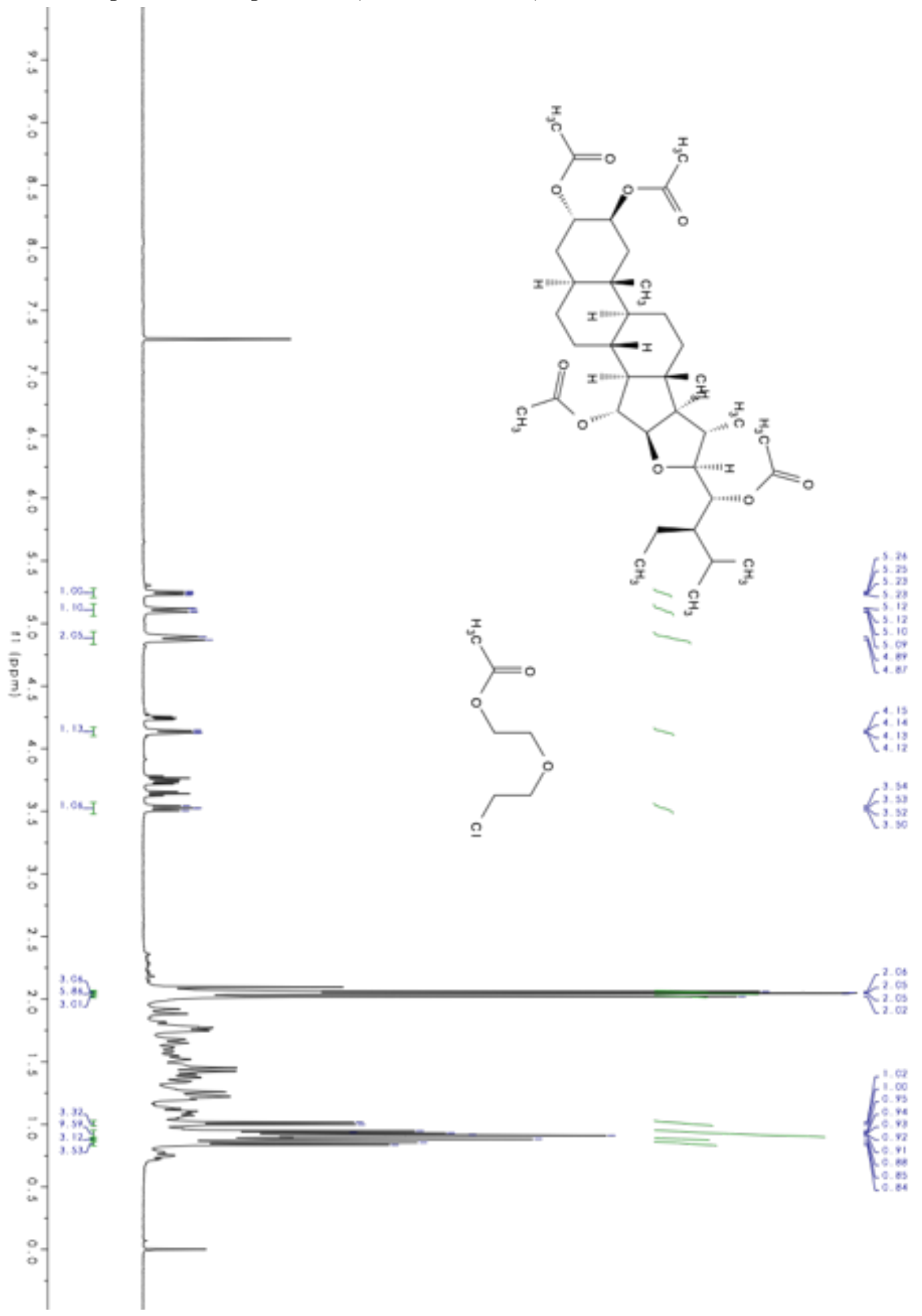


${ }^{13} \mathrm{C}$ NMR spectra of compound $28\left(100 \mathrm{MHz}, \mathrm{CDCl}_{3}\right)$

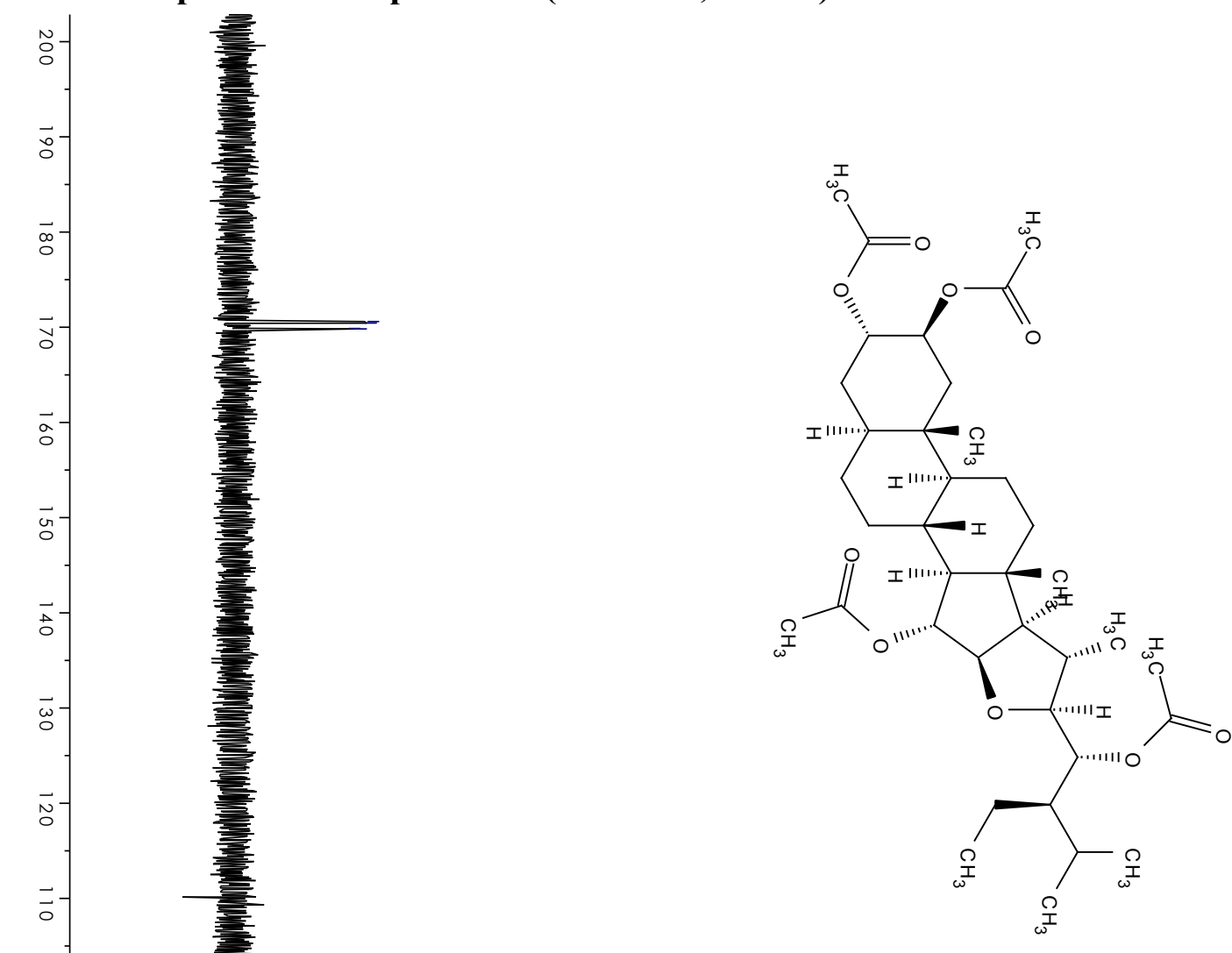

$\begin{array}{r}170.60 \\ -170.44 \\ \hline 169.87\end{array}$

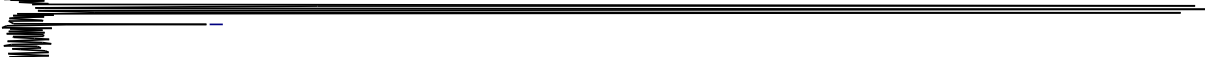

$-75.77$

(

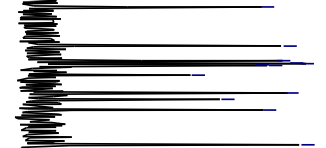

21.30

$\left[\begin{array}{l}21.23 \\ 20.35\end{array}\right.$

$\vec{\circ}$ 
${ }^{13} \mathrm{C}$ NMR DEPT spectra of compound $28\left(100 \mathrm{MHz}, \mathrm{CDCl}_{3}\right)$

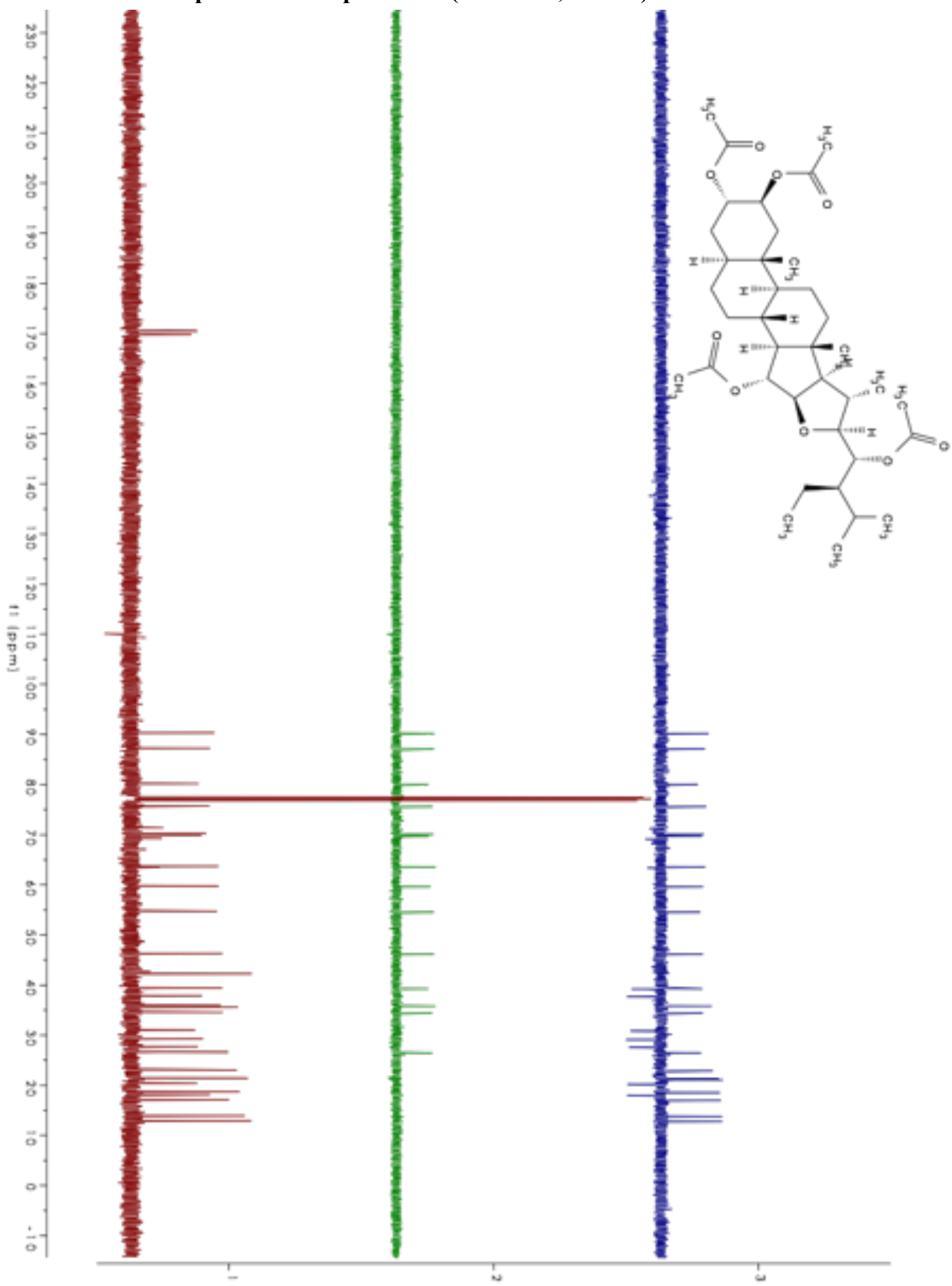


${ }^{1} \mathrm{H}-{ }^{1} \mathrm{H}$ NOESY spectra of compound $28\left(100 \mathrm{MHz}, \mathrm{CDCl}_{3}\right)$

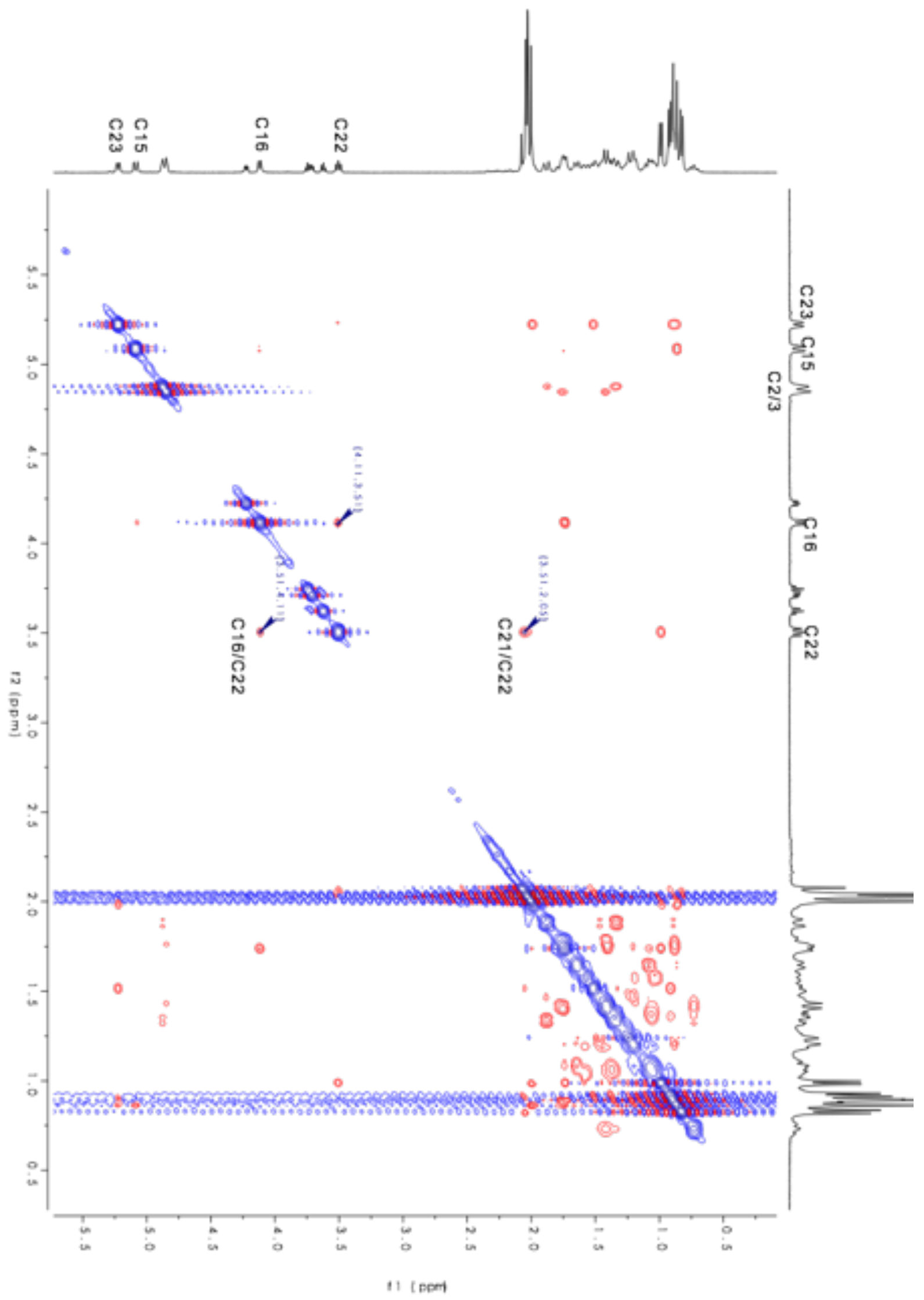


${ }^{1} \mathrm{H}$ NMR spectra of compound $29\left(400 \mathrm{MHz}, \mathrm{CDCl}_{3}\right)$

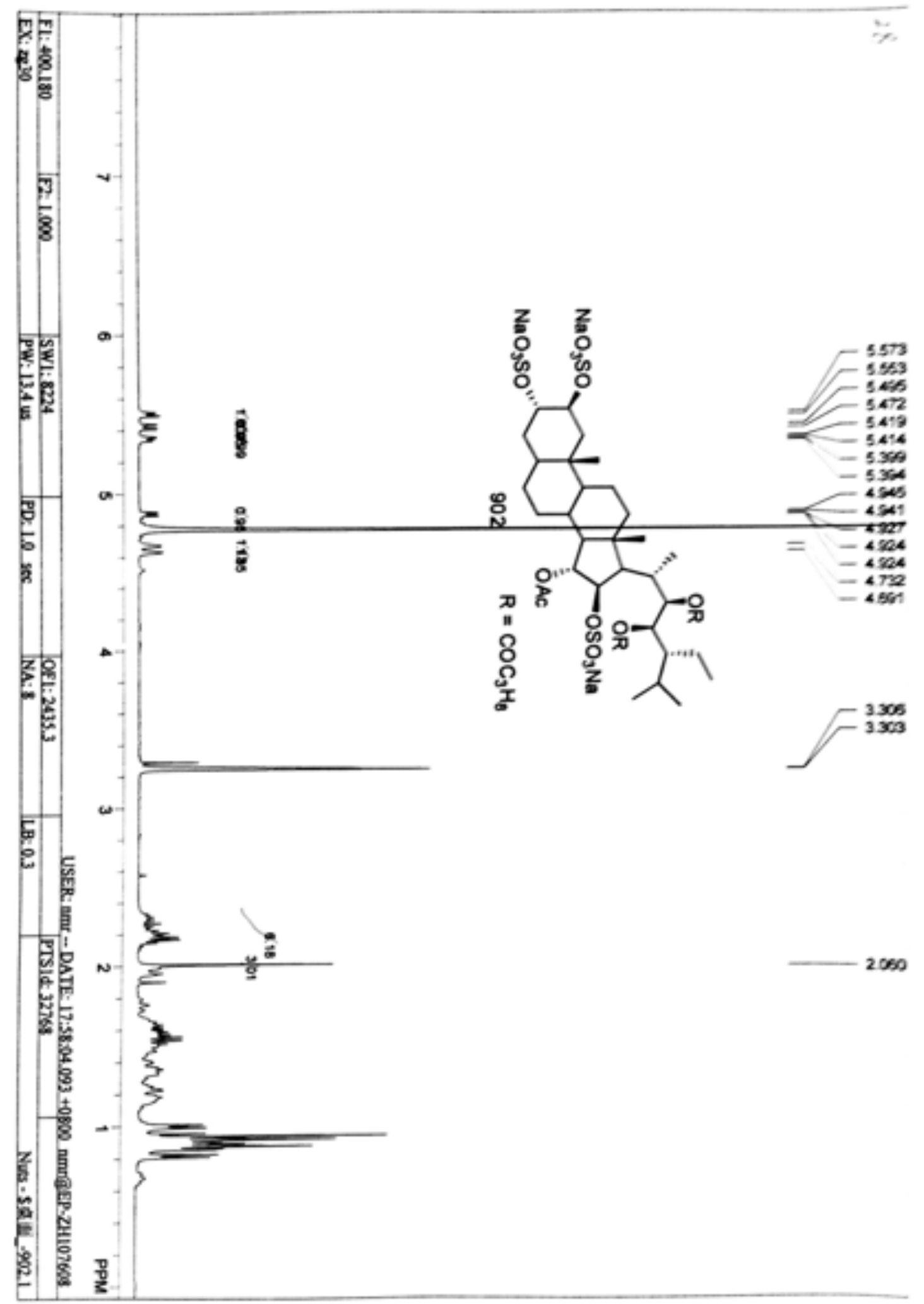


${ }^{13} \mathrm{C}$ NMR spectra of compound $29\left(100 \mathrm{MHz}, \mathrm{CDCl}_{3}\right)$

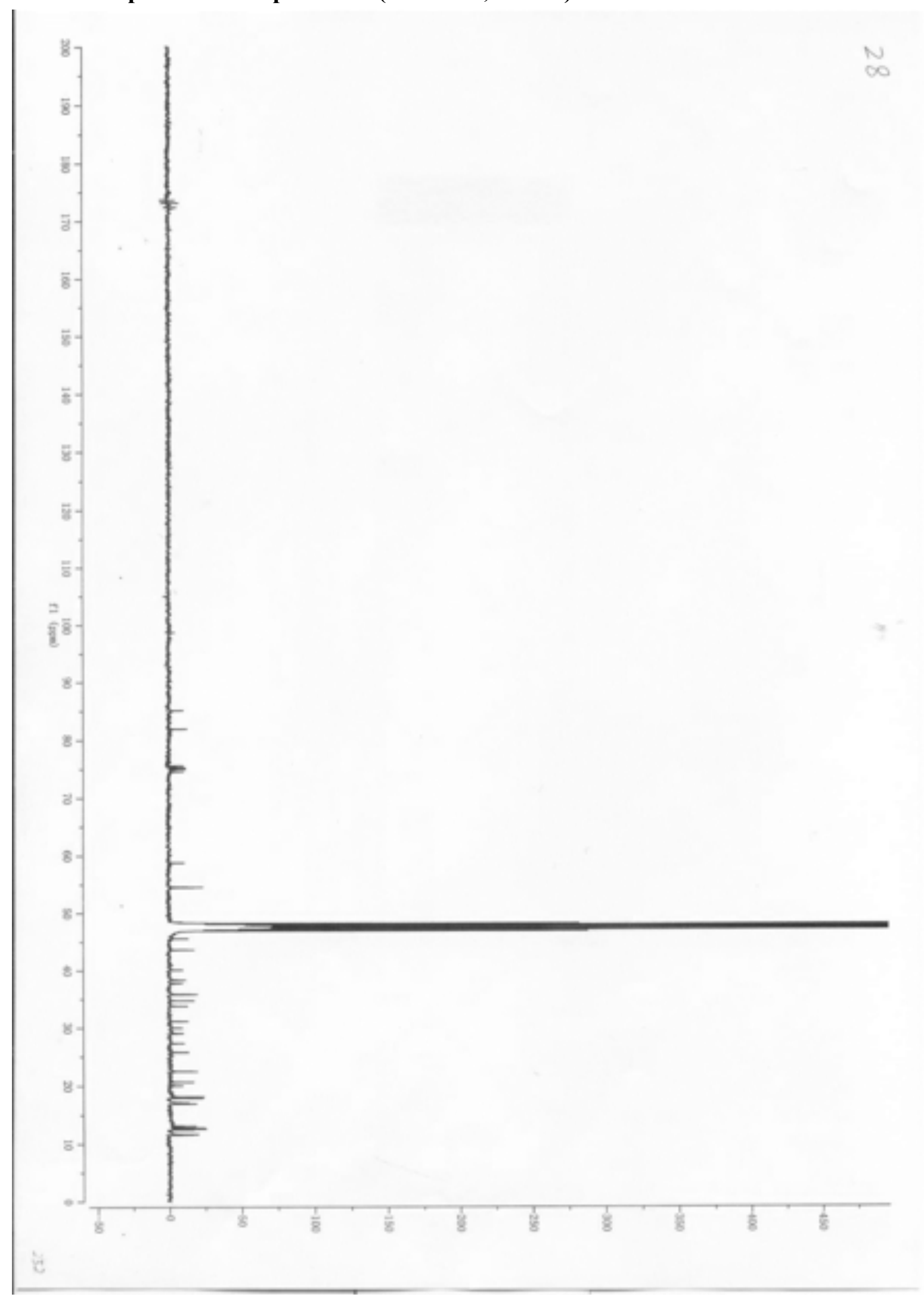

